Tao Hong

Transient Avalanche Oscillation of IGBTs Under High Current 



\section{Tao Hong}

\section{Transient Avalanche Oscillation of IGBTs \\ Under High Current}

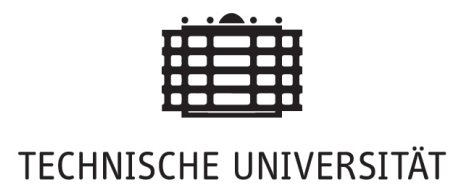

CHEMNITZ

Universitätsverlag Chemnitz

2015 


\section{Impressum}

\section{Bibliografische Information der Deutschen Nationalbibliothek}

Die Deutsche Nationalbibliothek verzeichnet diese Publikation in der Deutschen Nationalbibliografie; detaillierte bibliografische Angaben sind im Internet über http://dnb.d-nb.de abrufbar.

Technische Universität Chemnitz/Universitätsbibliothek

\section{Universitätsverlag Chemnitz}

09107 Chemnitz

http://www.tu-chemnitz.de/ub/univerlag

\section{Herstellung und Auslieferung}

Verlagshaus Monsenstein und Vannerdat OHG

Am Hawerkamp 31

48155 Münster

http://www.mv-verlag.de

ISBN 978-3-944640-52-5

http://nbn-resolving.de/urn:nbn:de:bsz:ch1-qucosa-164238 


\title{
Transient Avalanche Oscillation of IGBTs Under High Current
}

\author{
Doctoral Thesis \\ to obtain the academic degree of \\ Doctor of engineering (Dr.-Ing.)
}

submitted to

Faculty of Electrical Engineering and Information Technology at Chemnitz University of Technology

\author{
by Diplom-Ingenieur Tao Hong \\ born on 1971/07/09 in Hebei China
}

Date of submission: $14 / 10 / 2014$

Date of the defence: 11/03/2015

Examiner: Prof. Dr.-Ing. Josef Lutz

Co-examiner: Prof. Dr. phil. nat. D. Silber 



\section{Acknowledgements}

This work was conducted during my Ph.D contract with Infineon Technologies AG and as employee of the team called "Innovative Concepts". I thank Infineon for the opportunity to investigate the oscillations of IGBT Modules and the provision of laboratory conditions.

I would like to take this opportunity to thank Dr. Reinhold Bayerer. His wide overview of power modules has led me to the work field on high frequency oscillations in IGBTs. The fruitful technical discussion with him and his technical and organizational support made this work possible.

I would like to express my special thanks to Dr. Frank Pfirsch. His provision of basic structure of an IGBT cell facilitated my training in the device simulation. Through our discussions, I received numerous benefits from his professional experience on power semiconductors in fitting the IGBT device model regarding the forward and blocking characteristics.

I would like to express my gratitude to my supervisor Prof. Dr. Josef Lutz. He provides me the device simulation tools which are necessary for the analysis on the $\mathrm{HF}$-oscillation. His extensive expertise has relieved me from the search the state of the art. And I thank him for his great patience in the proofreading my thesis.

I would like to express my great appreciation to my second supervisor Prof. Dr. phil. nat. Dieter Silber, for the valuable technical discussions on space charge limited current flow and valuable information on the interaction of IMPATT and PETT.

I would like to thank Mrs. Susanne Fichtner and Dr. Thomas Basler, for their indispensable support during the incorporation of Sentaurus Device Simulator and in the ongoing maintenance of my simulation computer at the Chemnitz University of Technology. The material parameter set of silicon from Dr. Thomas Basler has saved me a lot of detours and time.

I wish to acknowledge the assistance on the familiarization in the 3.3 
kV IGBT oscillations provided by Mr. Thomas Gutt, Dr. Maria Cotorogea, Mr. Ronny Herms, Mr. Daniel Heer and Mr. Karl-Heinz Hoppe. And I also thank Dr. Markus Thoben for the methodical discussions.

Ms. Karin Schulze deserves my thanks for her assistance in both the patent registration and proofreading the German abstract of my thesis. I thank Dr. Han Hong, Mr. Rashedul Karim and Mr. William Etienne Akem for the proofreading English part this thesis.

Also, I thank all colleagues during my work at Infineon for their kind encouragement.

I wish to thank my parents and my brothers for their encouragements and cares in my life. Finally, I am immensely grateful for the essential support of my wife Hui Juan and the smiles of my Kids. 


\section{Contents}

$\begin{array}{ll}\text { Zusammenfassung } & 1\end{array}$

$\begin{array}{lr}\text { Abstract } & 2\end{array}$

1 Introduction 3

2 Functionality, turn-off and phenomenons of RF-oscillations in IGBT modules $\quad 7$

2.1 Functionality of modern IGBTs $\ldots \ldots \ldots$

2.2 Basic design of IGBT modules . . . . . . . . . . . 9

2.3 IGBT turn-off . . . . . . . . . . . . . . . . . . 11

2.4 Possible RF-oscillations during IGBT switching . . . . . . . 21

2.4.1 Tail oscillation PETT . . . . . . . . . . . . 21

2.4.2 Dynamic IMPATT oscillation . . . . . . . . . 25

2.4.3 Transient Avalanche oscillations . . . . . . . . . . . 29

3 Mechanisms of Transit time effect and RF-oscillations in IGBT Modules $\quad 31$

3.1 Amplification process . . . . . . . . . . . . 32

3.1.1 Avalanche breakdown . . . . . . . . . . . 33

3.1 .2 Amplification efficiency . . . . . . . . . . 35

3.2 Resonant circuits in IGBT modules . . . . . . . . . . . 40 
4 Analysis of Transient Avalanche oscillation 41

4.1 Measurement of TA-oscillations . . . . . . . . . . . 42

4.1.1 TA-oscillation on IGBT modules . . . . . . . . 42

4.1.2 TA-oscillation on a substrate and single chip . . . 48

4.2 Analysis of the resonant circuits . . . . . . . . . . 59

4.2 .1 Inductance of wire bonds . . . . . . . . . . 60

4.2.2 Differential capacitance of IGBTs and diodes . . . 63

4.3 Device simulations . . . . . . . . . . . . . . 65

4.3 .1 Circuit . . . . . . . . . . . . . 67

4.3.2 Effect of resonant circuit's eigenfrequencies . . . . 68

4.3 .3 Effect of load current . . . . . . . . . . . . 71

4.3.4 Effect of gate resistance . . . . . . . . . . 74

4.3.5 Internal process of IGBT chips . . . . . . . 75

4.3.6 Effect of impact ionization . . . . . . . . . . 76

4.4 Summarized explanation of TA-oscillations . . . . . . 80

5 Countermeasures to Transient Avalanche oscillations 83

5.1 Essential measures

to suppress TA-oscillations . . . . . . . . . . . 83

5.2 Driver concept to suppress TA-oscillations - pace maker . . 85

6 Summary and outlook $\quad 91$

6.1 Summary ......................... 91

6.2 Outlook . . . . . . . . . . . . . . . 94

$\begin{array}{ll}\text { A Derivation of transit time efficiency } & 97\end{array}$

$\begin{array}{ll}\text { B Test box and the RF-noise problems } & 101\end{array}$

$\begin{array}{ll}\text { Bibliography } & 103\end{array}$ 


\section{List of symbols}

$\alpha_{e f f}$ effective ionization coefficient $1 / \mathrm{cm}$

$\alpha_{n, p} \quad$ ionization coefficient of electrons, holes $\quad 1 / \mathrm{cm}$

$\epsilon \quad$ relative permittivity

$\begin{array}{lll}\epsilon_{0} & \text { vacuum permittivity } & \mathrm{As} / \mathrm{Vm}\end{array}$

$\eta \quad$ efficiency coefficient of the RF-power conversion -

$\eta_{1} \quad$ injection dependent efficiency coefficient -

$\eta_{2} \quad$ transit time dependent efficiency coefficient -

$\lambda \quad$ proportionality between $N_{h_{-} \text {nett }}$ and $I_{C E}$

$\bar{v}_{d n, d p}$ effective velocity of electrons, holes $\mathrm{cm} / \mathrm{s}$

$\Theta_{D} \quad$ transit angle

$\Theta_{M} \quad$ phase angle of the injection -

$\Theta_{W} \quad$ injection angle

$v_{d n, d p}$ drift velocity of electrons, holes $\mathrm{cm} / \mathrm{s}$

$v_{Q} \quad$ velocity of charge carrier $\mathrm{cm} / \mathrm{s}$ 
A chip area $\mathrm{cm}^{2}$

$C_{D C} \quad$ DC-link capacitor $\quad F$

$C_{\text {diff }}$ effective capacitance in RF-range $\quad F$

$C_{\text {Diode }}$ differential capacitance of SCR in diode $\quad F$

$C_{I G B T}$ differential capacitance of SCR in IGBT $\quad F$

$E_{C} \quad$ critical E-field strength $\quad \mathrm{V} / \mathrm{cm}$

$E_{\text {max }}$ maximal E-field in the space charge region $\quad \mathrm{V} / \mathrm{cm}$

$I \quad$ current $A$

$I_{L} \quad$ load current, trun-off current of IGBT $A$

$I_{N}$ norm current $\quad \mathrm{A}$

$I_{w b} \quad$ load current in the wire bonds $A$

$J_{D C} \quad$ induced average current density $\quad \mathrm{A} / \mathrm{cm}^{2}$

$J_{\text {ind }}$ induced current density on the terminal $\mathrm{A} / \mathrm{cm}^{2}$

$l \quad$ coil length $\quad \mathrm{mm}$

$L_{a} \quad$ inductance of the avalanche region $\quad H$

$L_{B 1, B 2}$ inductance of wire bonding $\quad H$

$L_{l c, s c}$ inductance of long, short coil $\quad H$

$L_{P} \quad$ load inductance $\quad H$

$L_{w b} \quad$ inductance of wire bondings $\quad H$

$N_{A} \quad$ density of the acceptors $\quad 1 / \mathrm{cm}^{3}$

$N_{D} \quad$ density of donors $1 / \mathrm{cm}^{3}$ 
$N_{e_{-} \text {Plasma }}$ electron-density in plasma

$1 / \mathrm{cm}^{3}$

$N_{e} \quad$ electron-density

$1 / \mathrm{cm}^{3}$

$N_{h \_n e t t}$ net hole-density

$1 / \mathrm{cm}^{3}$

$N_{h}$ Plasma hole-density in plasma

$1 / \mathrm{cm}^{3}$

$N_{h} \quad$ hole-density

$1 / \mathrm{cm}^{3}$

$P_{D C}$ power consumption of DC voltage source

$P_{R F} \quad$ radio frequency power generation

$Q \quad$ injection charge

$r_{c} \quad$ radius of coils

$m m$

$R_{B 1, B 2}$ resistance of wire bonding

$R_{g} \quad$ gate resistance

$R C_{1,2,3}$ resonant circuits

$t \quad$ time

$T_{D} \quad$ transit time

$T_{i} \quad$ injection time

$t_{\text {on }}$ on time

$V_{D C} \quad$ DC-link voltage

$v_{e_{-}}$Plasma electron-velocity in Plasma $\mathrm{cm} / \mathrm{s}$

$V_{G E} \quad$ gate-emitter voltage

$v_{h}$ Plasma hole-velocity in Plasma

$V_{R F} \quad$ amplitude of the AC voltage 
$V_{T} \quad$ terminal voltage on the transit time structure

$W_{A} \quad$ width of the avalanche region $\quad \mathrm{cm}$

$W_{c} \quad$ field energy of coils $\quad W$

$W_{S C R}$ width of the space charge area $\mathrm{cm}$

$W_{w b} \quad$ field energy of emitter wire bondings $\quad W$ 


\section{Zusammenfassung}

Radio Frequenz Oszillationen bis zu mehreren hundert $\mathrm{MHz}$ wurden während des Abschaltens von Hochstrom leitenden IGBTs beobachtet. Sie werden in dieser Arbeit als Transient Avalanche Oscillationen beschrieben. Anders als PETT Oszillationen, die in der Tail-Strom Phase vorkommen, erscheint diese Oszillation während des Anstiegs der Kollektor-EmitterSpannung $V_{C E}$ und des Fallens des Kollektor-Emitter Stroms $I_{C E}$. In dieser Arbeit werden die Abschaltvorgänge von IGBTs mit Hilfe von Messungen, Schaltungs- bzw. Device-Simulationen untersucht, um die Wirkungsmechanismen der transient erscheinenden hochfrequenten Oszillationen zu erklären. Es ist erstmals gelungen solche Transient Avalanche Oszillationen mit der Simulation zu reproduzieren und anschließend die Einflüsse einzelner Parameter zu untersuchen. Die Beteiligung des IMPATT Mechanismus an der Transient Avalanche Oszillation wurde nachgewiesen. Die Interaktion von IMPATT und PETT während Aufbau der Raumladungszone im IGBT wurde untersucht. Maßnahmen zur Unterdrückung der Oszillation werden auf den Ebenen von Chip-Design, Modulauslegung und der Treiberschaltung diskutiert. Das Zuführen des Elektrostromes während der kritischen Phase wird als ein effective Methode zur Vermeidung der TAOscillation nachgewiesen.

IGBT, Abschalten, Oszillation, Transient Avalanche, Resonanz, Raumladung, IMPATT, PETT, TA-Oszillation 


\section{Abstract}

Radio frequency oscillations up to several hundreds of $\mathrm{MHz}$ were observed during turn-off the high-current conducting IGBTs. They are described in this work as Transient Avalanche oscillations. Other than PETT oscillations that happen in the tail current phase, this oscillation appears during the rise of collector-emitter voltage $V_{C E}$ and during the fall of the collectoremitter current $I_{C E}$. The turn-off process of IGBTs are investigated with the help of measurements, the circuit- and device-simulations to reveal the working mechanisms of transient high-frequency oscillations. For the first time the Transient Avalanche Oscillation is successfully reproduced with computer simulation, which allows the further investigation on the influences of individual parameters. The participation of IMPATT mechanism in Transient Avalanche oscillation is demonstrated. The interaction between IMPATT mechanism und PETT mechanism during the setting up of depletion region in IGBTs was investigated. Measures to suppress such oscillation are discussed on the levels of chip design, module design and the driver concept. Supplying electron current during critical phase is found to be an effective method to avoid the dynamic avalanche and the TA-oscillation.

IGBT, turn-off, Oscillation, Transient Avalanche, Resonance, Space charge, depletion region, IMPATT, PETT, TA-oscillation 


\section{Chapter 1}

\section{Introduction}

In addition to the reliability, higher blocking capability, lower conduction losses, lower switching losses and higher switching frequency are the basic requirements for designing power semiconductors. The approach to improve such properties by combining the advantages of MOSFET (metaloxide-semiconductor field-effect transistor) and BJT (bipolar transistor) was reported in the late seventies [Bal79]. In 1980 the U.S. Patent Office granted a U.S. patent "Power MOSFET with an anode region" with patent No. 4,364,073 to Hans W. Becke and C. Frank Jr. Wheathley [BW80]. This patent is regarded as the invention of the insulated gate bipolar transistor, briefly called IGBT, which merged BJT and MOSFET technically.

After the development over three decades, more and more approaches have been developed by advantageous combination of bipolar and unipolar elements. Up to date, IGBT finds wider and wider application fields. Parallel to the industrial motor control and inverters of renewable energy, IGBTs are also widely used in traction applications, auto mobiles and consumer electronics. The application area of IGBTs extends even to the typical application area of thyristors with the development of high power 
IGBTs. The first $3.3 \mathrm{kV}$ IGBT Module was developed by Eupec GmbH in 1995. The first $6.5 \mathrm{kV}$ IGBT module was developed also by Eupec $\mathrm{GmbH}$ in $1999\left[\mathrm{ABG}^{+} 99\right]$. The development of $8 \mathrm{kV}$ IGBT and diode chips was reported in $2002\left[\mathrm{RKK}^{+} 02\right]$. Up to date the $6.5 \mathrm{kV}$ IGBT modules are still the commercially available Modules with maximal blocking capability.

One important aspect of the IGBT development focuses on the reduction of conduction and switching losses and to increase the current density on silicon. To fulfil increasing demands on the electrical and thermal performance in high power applications, IGBTs and diodes are paralleled and integrated in modules. In the modules, IGBT chips work in a complicated parasitic environment which consists of IGBT capacitance, diode capacitance, parasitic inductances and capacitances as a consequence of the module construction. Such parasitic components build up resonant circuits. In the dynamic process, such as turn-on and turn-off, the resonant circuits can be excited which cause undesirable oscillations. Especially the high-frequency oscillations can cause electromagnetic interference for the complete system.

Under a special design of a module, high frequency oscillations of up to several hundreds of megahertz are observed during turn-off of the IGBT. This oscillation phenomena has been found in the tail current phase of NPT IGBTs in flat package [TYK $\left.{ }^{+} 97\right]$ and was explained by the the mechanism of plasma extraction transit time (PETT) oscillation [GMS01] [GMS02] [SLNM03]. The PETT oscillations were usually observed during the tail current. After the IGBT has taken the reverse voltage, there is still electron-hole plasma remaining close to the collector side. This residual plasma is extracted out of IGBT by the tail current. The holes drift through space charge region $(S C R)$ under the influence of the strong electric field to the emitter side and recombine with electrons. The holes need a certain time to be injected in to the space charge region and a transit time to drift through the space charge zone. Such injection time and the 
transit time together induce a phase shift between terminal voltage and the terminal current in the RF-frequency range. For a certain frequency range the phase shift lies in the interval between $1 \pi / 2$ and $3 \pi / 2$. The generates therefore power and works in this frequency range as an amplifier. This BARITT-diode similar mechanisms (barrier injection transit-time diode) [Roe77] [Sze81] was analysed in details in [Gut06] and [SMNL06]. RFfrequency oscillations has also been found during the turn-off of fast recovery diode. The temporarily charged K-defects, radiation induced deep levels, leads to the temporary reduction of the avalanche breakdown voltage and lead to the dynamic IMPATT (Impact Ionization Avalanche Transit Time) oscillation during diode turn-off [LSG98] [Lut00] [SLH04]. Both IMPATT and PETT oscillations lead to deterioration of EMC and should be avoided. consequently [SLNM03].

Recently a new type of high-frequency oscillation was found during the turn-off of IGBTs. It happens in the dynamic avalanche [SN89] [DLS03] phase during the rise of the collector-emitter voltage and the fall of the collector-emitter current. At the moment of oscillation, the load current or a large portion of the load current is still flowing. Since this oscillation is observed only transiently and only during the dynamic avalanche process,

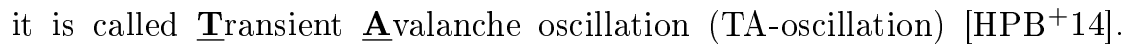
The mechanisms of this oscillation seems to be closely related to the process found in IMPATT diode [EH98]. The target of this work is to reveal its mechanism and to figure out the countermeasures to prevent or suppress such TA-oscillations.

In Chapter 2, the construction and characterizations of IGBTs are shown. The IGBT turn-off process is described according to the internal semiconductor states. Finally, the state of art mechanisms of PETT oscillation and the dynamic IMPATT oscillation is represented.

In Chapter 3, the theoretical analysis for the transit-time effect and mechanisms of IMPATT- and BARITT-diode are summarized. The anal- 
ysis of the efficiency of the transit-time effect in the space charge zone of the IGBTs results in the basic of theoretical explanation of the TA oscillation. Whereas efficiency means the efficiency to amplify RF-oscillations by the TA-effect.

The detailed investigation of the TA-oscillation by means of measurements, resonant circuit analysis and simulations are presented and followed by the theory of the TA-oscillation in Chapter 4 .

In chapter 5, the countermeasures of TA-oscillation are investigated, and a new driver concept is introduced to suppress TA-oscillation.

The summary in chapter 6 provides an overview of the HF-frequency oscillations in power modules, that is followed by the outlook on the further possibilities to suppress such oscillation by methods of module design and chip designs. 


\section{Chapter 2}

\section{Functionality, turn-off and}

phenomenons of

RF-oscillations in IGBT

\section{modules}

To analyse the TA-oscillation mentioned in Chapter 1, it is necessary to understand the functionality of IGBT and to describe the turn-off process with the internal semiconductor states. A hypothesis on the mechanisms of TA-oscillation can be set up based on the results of the investigation on the relationships between IGBT-, diode-chips and parasitic components of the IGBT modules constructions. 


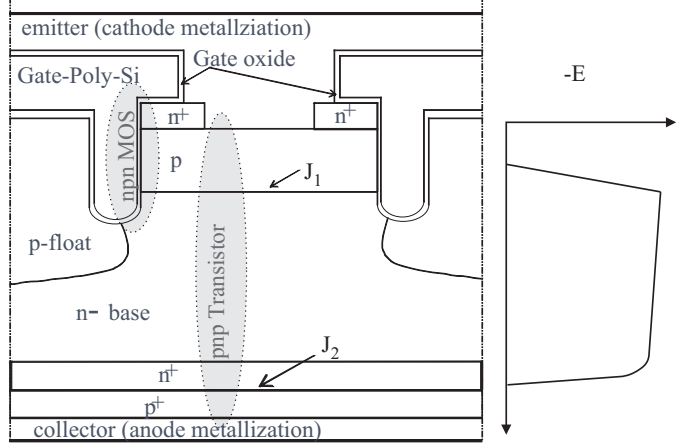

Figure 2.1: Cell structure of a modern trench IGBT with field stop on the collector side [Hon14].

\subsection{Functionality of modern IGBTs}

An IGBT works essentially like a MOS-channel controlled bipolar Transistor. The cell structure of a modern trench IGBT with field stop on the back side is illustrated schematically on the left side of Fig. 2.1. The cathode (IGBT emitter) is on the top of the chip, and the anode (IGBT collector) is on the bottom. The electrical contacts to the outside circuit are usually realized by the metallizations on the silicon body. A highly doped polysilicon layer serves as a gate electrode. The MOS-channel is controlled by the voltage at the gate. Due to the positive voltage between collector and emitter, the junction $\mathrm{J}_{1}\left(\mathrm{n}^{-} \mathrm{p}\right)$ on the emitter side is polarized in the reverse direction. The junction $\mathrm{J}_{2}\left(\mathrm{p}^{+} \mathrm{n}^{+}\right)$on the collector side is polarized in the forward direction. In the blocking state, the gate-emitter potential is generally set to a voltage between $0 \mathrm{~V}$ and $-15 \mathrm{~V}$ to keep the MOS-channel off. A space charge region is built around $\mathbf{J}_{1}$ and extends far into the low doped $\mathrm{n}^{-}$type base region. With the increasing reverse voltage, the space charge region reaches the $\mathrm{n}^{+}$layer of $\mathrm{J}_{2}$, where the heavy doping makes a high electric field gradient. Thus a trapezoidal E-field distribution is formed as shown on the right side of Fig. 2.1. Currently, depending on 
the thickness of the base region IGBTs can be commercially designed for a blocking capability up to $6.5 \mathrm{kV}$.

In forward operation, the gate-emitter voltage is usually switched to $+15 \mathrm{~V}$. The MOS-channel is turned on. Electrons flow through the MOS channel and $\mathrm{n}^{-}$base region to junction $\mathrm{J}_{2}$ on the collector side. Similar to a bipolar transistor, holes from $\mathrm{p}^{+}$are injected into the $\mathrm{n}^{-}$base. Due to the wide $\mathrm{n}^{-}$base of the PNP-transistor (see Fig. 2.1), the current gain of the transistor is less than one [Sil05]. That is, its base current is greater than the collector current. As the PNP transistor gets into the on-state, holes get from the $\mathrm{p}^{+}$layer (collector) in the $\mathrm{n}^{-}$base. The base is than flooded with an electron-holes plasma und gets in the state of high injection. This results in a good conductivity in the forward operation of the IGBT. The hole current flow is suppressed by the deep trench gate geometry. The injection efficiency of holes near to $\mathrm{J}_{1}$ at the emitter side is therefore enhanced. A plasma distribution similar to a thyristor can be realized due to this injection enhancement effect [ $\mathrm{KOH}^{+} 93$ ] [OOSO97]. Compared to the planar and trench IGBT, as shown in Fig. 2.2, the combination of field stop layer and trench cell design results in the optimized carrier distribution, the minimum forward voltage drops and the minimum switching losses $\left[\mathrm{LMP}^{+} 00\right]$. Further analysis in this work will be carried out using this cell structure.

\subsection{Basic design of IGBT modules}

Packaging is generally an important topic of semiconductor industry. On one side, the package offers mechanical protection to the chips. On other side, the chips are thereby electrically and thermally connected to the outside. In the applications, a power circuit always consists of several components to satisfy the electrical and thermal requirements. Integration of IGBTs and diodes into power modules facilitate the design of power 


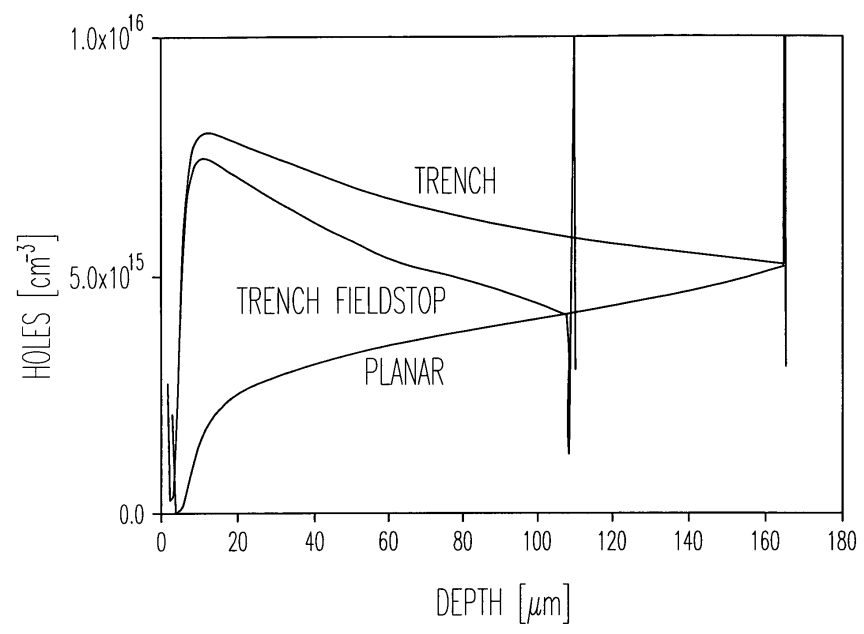

Figure 2.2: Plasma distributions of Planar-IGBT, Trench-IGBT and Trench FS-IGBT $\left[\mathrm{LMP}^{+} 00\right]$ [R.B10]

electronics.

DCB-substrates (direct copper bounding) are frequently used as the circuit substrate in the IGBT modules where two copper layers are bonded on each side of a ceramic sheet. Fig. 2.3a. shows the construction of such a substrate equipped with IGBT and diode chips. IGBT- and diode-chips are bonded on the top side of a DCB. The top side copper layer of the DCB serves as the circuit carrier. The ceramic and the bottom copper layer of the DCB ensure the electrical insulation and heat conduction to the heat sink. Wire-bonds are applied to realize the electrical connection between the top side of the chips and electrical terminals on the DCB. Fig. 2.3b. shows an half bridge circuit. The circuit consists of two IGBTs connected in series and their anti-parallel free-wheeling diodes.

Besides the desired electrical and thermal connections, the module structure results in inevitable parasitic effects. The parasitic electrical resistances and inductances of the bond wires, parasitic capacitance within 
the substrate are some main parasitic components of a module structure. The equivalent circuit of an IGBT module, as can be seen in Fig. 2.3c, is therefore much more complex than the circuit in Fig. 2.3b. The circuits for parallel connected dies are even more complex than Fig. 2.3c. The parasitics of the module structure together with the semiconductor chips may constitute resonant circuits which are excited during switching processes resulting in oscillations. This leads to significant deviation from the ideal clean switching [BD10]. The switching performances is worthened by such oscillations. Further investigation of the resonant circuit will be addressed in Chap.4.2.

\subsection{IGBT turn-off}

To bring the IGBT from the conducting state to the blocking state, the plasma must be extracted out of the base region. This extraction process determines the dynamic behaviour of the IGBT. Fig. 2.4 shows the circuit setup applied for the dynamic characterization of the IGBT modules. During the dynamic test of the bottom IGBT, the top IGBT of the half bridge is kept off by a gate-emitter voltage $V_{G E_{-} \text {off }}$ between $0 \mathrm{~V}$ and$15 \mathrm{~V}$. The anti-parallel diode in the upper leg of the half bridge acts as the free-wheeling diode. The load inductance is connected parallel to the Top IGBT. A capacitor bank (so-called DC-link $C_{D C}$ ) is connected across the half bridge. $C_{D C}$ acts as an ideal voltage source for a short time. When the DUT is open ( $V_{G E}$ of the DUT is driven to $V_{G E}{ }_{o} f f$, the DUT is therefore blocking). The terminal voltage $V_{C E}$ on the DUT is equal to $V_{D C}$. And there is no current in the load inductance $L_{P}$. After the DUT is turned on, $V_{G E}$ is switched to $V_{G E_{-} \text {on }}$ (typically $+15 \mathrm{~V}$ ), the current in the $L_{P}$ and in the DUT increases. The load current $I_{L}$ can be adjusted by load inductance $L_{P}$ and the time $t_{\text {on }}$ according to Eq.(2.2). In order to bring the IGBT to a stationary flooded condition, $t_{o n}$ is generally adjusted to 


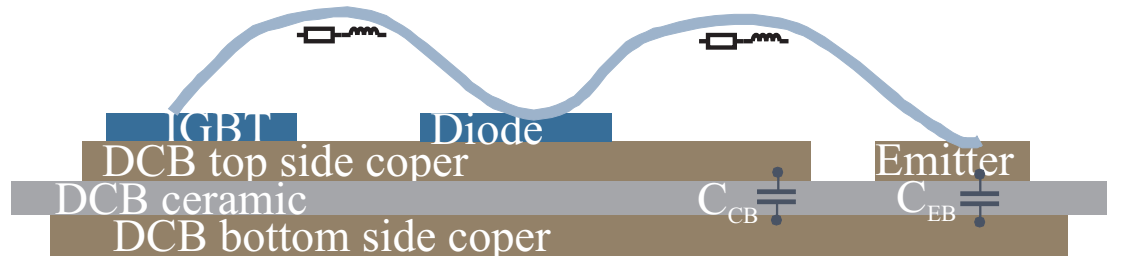

a. Construction of a substrate in IGBT modules

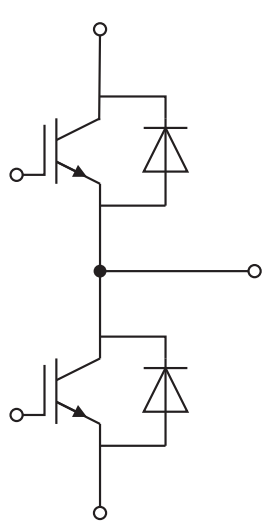

b. Ideal circuit of a half bridge

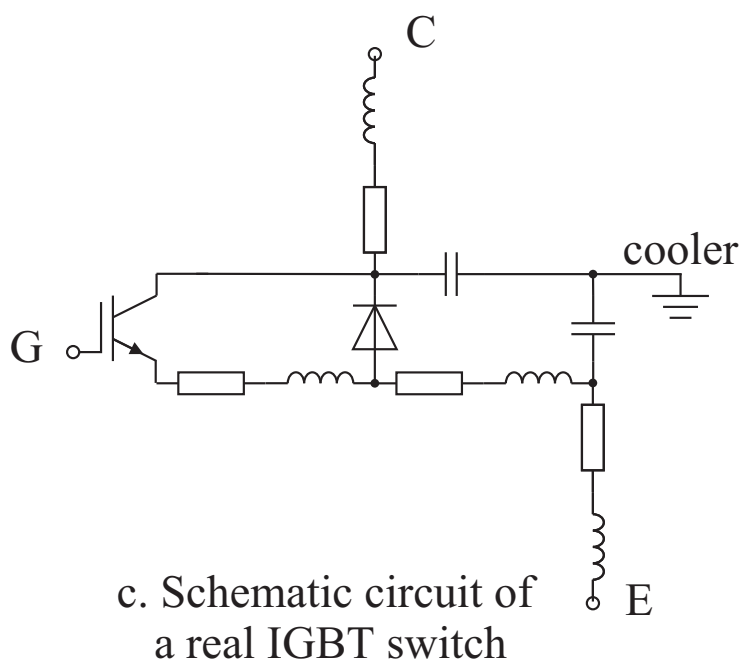
a real IGBT switch

Figure 2.3: a. ideal diagram of a IGBT module, b. Substrates with DCB structure and bonding wire, c. Equivalent circuit of a real IGBT module 


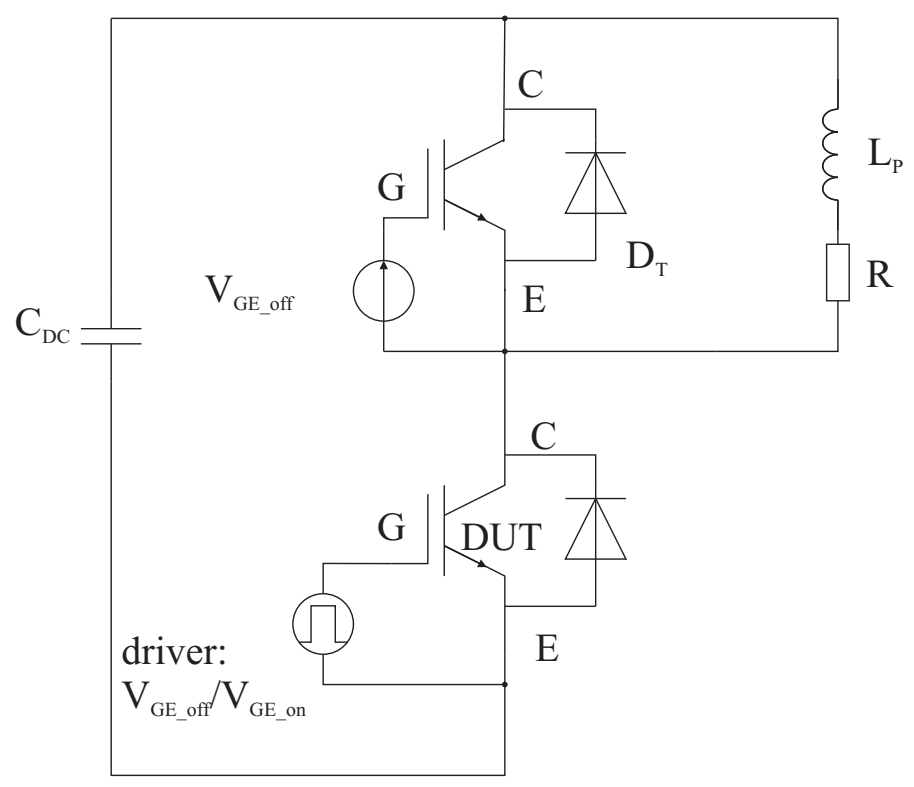

Figure 2.4: Circuit setup of the dynamic test 
more than $100 \mu \mathrm{s}$.

$$
\begin{aligned}
\frac{d I_{L}}{d t} & =\frac{V_{D C}}{L_{P}} \\
I_{L} & =t_{o n} \frac{V_{D C}}{L_{P}}
\end{aligned}
$$

With a forward current up to two times of the rated current $\left(2 \times I_{N}\right)$ , the IGBT is still in the IGBT-saturation ${ }^{1}$. The base region is flooded with a plasma of electrons and holes and the basic region is electrically neutral. Since the charge carrier density in the plasma is several orders of magnitude over the base doping, a weak E-field is sufficient to drive the load current passing through the base region. Fig. 2.5 presents the charge distribution in the base region schematically. Due to the in the weak E-field in the plasma, the mobility of electrons is about three times higher than that of holes. The ratio of electron-current to the hole-current should be roughly $3 / 1$. On the emitter-side only holes are deduced over $J_{1}$. The total electron-current is delivered by MOS channel.

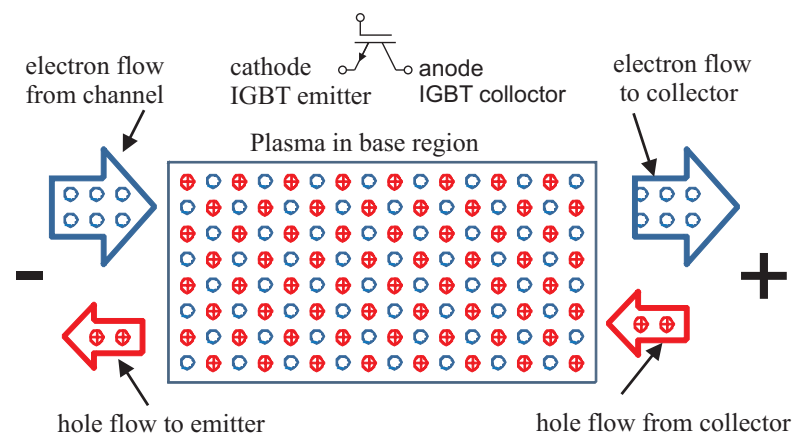

Figure 2.5: Schematic of plasma and carrier-flows in the stationary on state of an IGBT

\footnotetext{
${ }^{1}$ IGBT-saturation describes a state of the IGBT in which the charge carriers in the IGBT base region are ample and the forward current increase strongly with the forward voltage.
} 


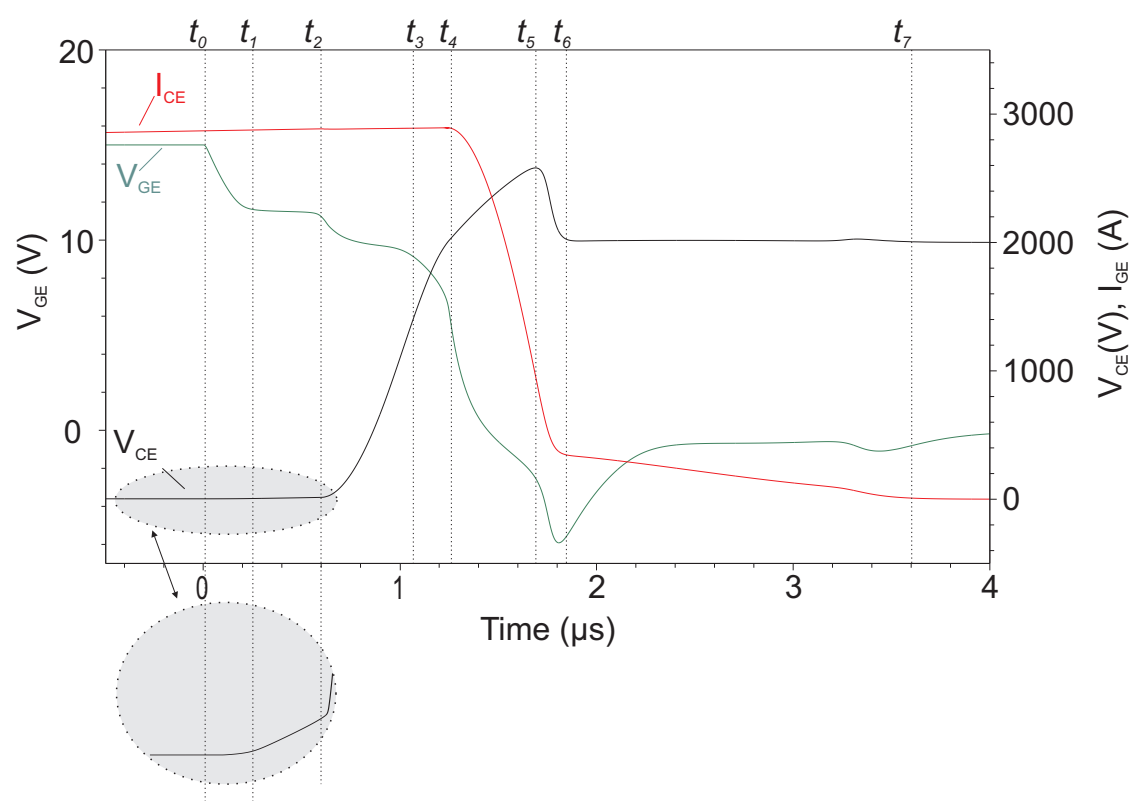

Figure 2.6: Turn-off diagram of a $3.3 \mathrm{kV}$ Trench Field Stop IGBT (simulation)

Fig. 2.6 represents a turn-off diagram of a $3.3 \mathrm{kV}$ Trench Field Stop IGBT. The turn-off process begins with the switching off the driver voltage. The driver voltage is switched from $+15 \mathrm{~V}$ to $0 \mathrm{~V}$ at the time $t_{0}$. Similarly to a MOSFET, the discharging of the gate capacitance drives the gate voltage first down to the Miller-plateau. In such period, the conductivity of the MOS-channel and $V_{C E}$ is kept nearly unchanged. On the Miller plateau, from $t_{1}$ to $t_{2}$, the MOS channel is in the MOS-saturation ${ }^{2}$. Due to the reduction of the channel conductivity, $V_{C E}$ increases by a few volts. The enlarged part below in Fig. 2.6 shows a linear increase of about 10V. The major portion of this voltage drops on the MOS channel. The reduction of the channel conductance can be thereby compensated and the channel

\footnotetext{
${ }^{2}$ MOS-saturation describes a state of the MOS-channel, in which the conductivity is sensitively dependent on the gate voltage.
} 
current is maintained. Because of the Miller capacitance, the feedback of $V_{C E}$ on the gate is so strong that the $V_{G E}$ remains almost constant in this period. Such behaviour can also be found on the Miller-plateau during turn-off of a MOSFET (Fig. 2.7). The channel current vanished with further discharging the gate capacity.

From time $t_{2}$, the IGBT passes to the active-state ${ }^{3}$. The channel current begins to decrease with the gate voltage. The sinking of channel current means less incoming electrons from the emitter side to the base region. Because $V_{C E}$ is still below the $V_{D C}$, the load current can not be commutated to the free-wheeling diode. The total load current must be carried by the IGBT. On the emitter side the missing electron-current must be taken over by holes. The electrical neutrality under the $J_{1}$ is broken. A layer of space charge with a net holes density of $N_{h_{-}}$net appears in the vicinity of $J_{1}$. The space charge region $S C R$ is formed and expands with the time to the collector. Poisson-equation (2.3) describes the dependence between the electric field and charge density in the $S C R$. Since the charge carrier density is much higher than the base doping, in one dimensional-case Eq.(2.3) can be expressed to Eq.(2.4).

$$
\frac{\partial E}{\partial x}=\frac{q}{\epsilon \epsilon_{0}}\left(N_{D}-N_{A}+N_{h}-N_{e}\right)
$$

with $N_{h \_n e t}=N_{h}-N_{e} \gg N_{D}-N_{A}$

$$
\frac{d E}{d x}=\frac{q}{\epsilon \epsilon_{0}} N_{h-n e t}
$$

With the modern computer technology, the essential semiconductor equations can be numerically solved by the Device Simulators to represent the

\footnotetext{
${ }^{3}$ In the active-state of a IGBT, the channel current is linearly dependent on the gate voltage.
} 


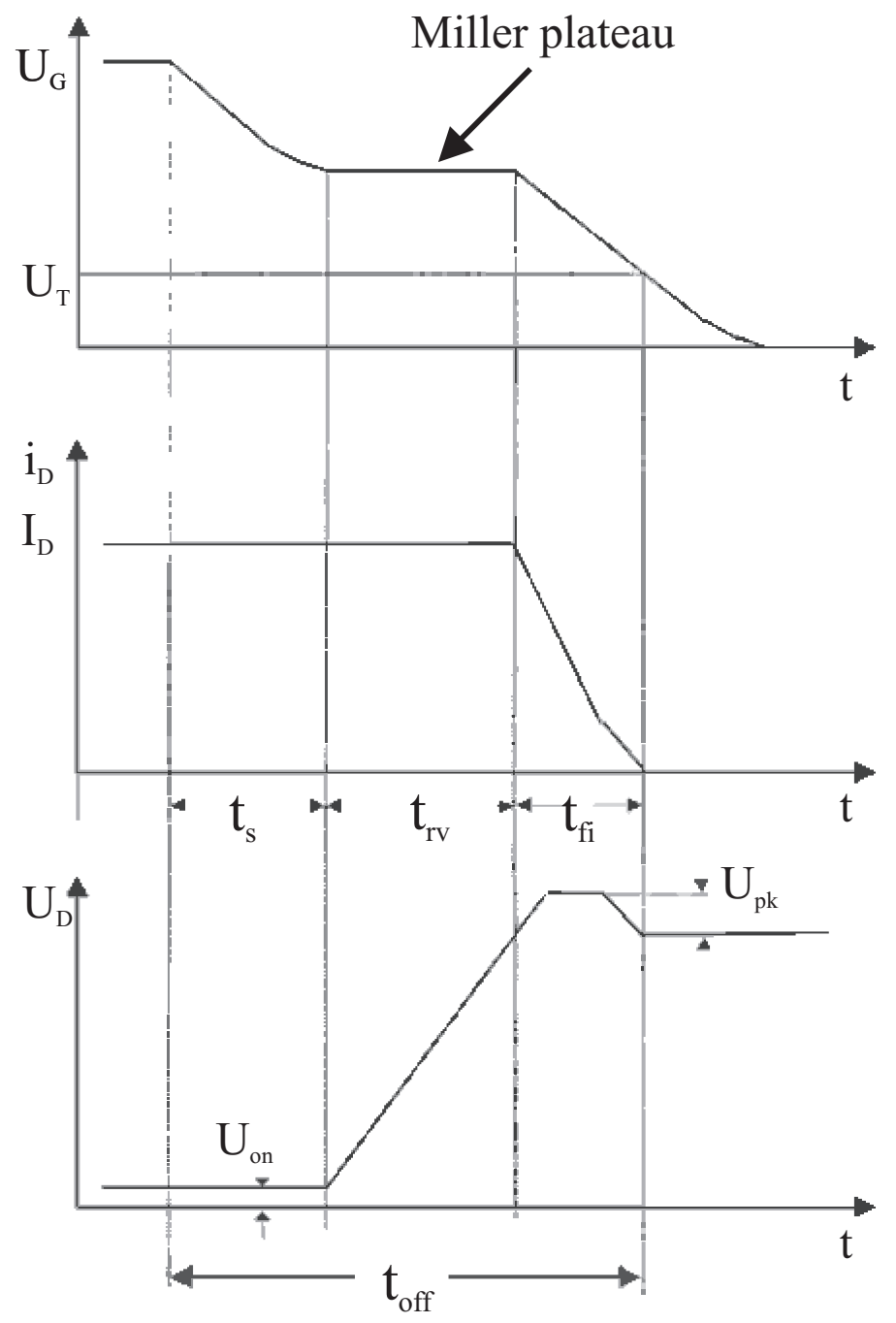

Figure 2.7: Turn-off process of a MOSFET with inductive load (Fig.9.17 [LSSD11]). At the time $t_{r v}$ the MOSFET is on the Miller-plateau. 
dynamic processes in semiconductors. Nevertheless the following analytical assessment is often useful to better understand the processes.

During the turning-off of IGBTs, the building-up of $S C R$ can be analytically derived under the following assumptions and simplifications approximately.

- Homogeneous charge distribution and neutrality in the plasma with the charge density $N_{\text {Plasma }}=N_{h_{-} \text {Plasma }}=N_{e_{-} \text {Plasma }}$

- Homogeneous charge distribution and triangular E-field distribution in main part of $S C R$

- In the plasma the electron velocity $v_{e_{-}}$Plasma is three times higher than that of the holes $v_{h_{-}}$Plasma.

$$
I_{C E}=A q N_{\text {Plasma }}\left(v_{h_{-} \text {Plasma }}+v_{e_{-} \text {Plasma }}\right)
$$

\section{A: chip area}

With $v_{e_{-} \text {Plasma }}=3 v_{h_{-} \text {Plasma }}$ the velocity of the electrons in the plasma can be represented as a function of load current and plasma density. The $S C R$ extends to the collector with the velocity $v_{e_{-}}$Plasma. After the turnoff of the channel current the neutral plasma front drifts toward the collector with the velocity of $v_{e_{-}}$Plasma. For the same IGBT, higher load current would lead to higher electron velocity in the plasma during the on-state and therefore leads to the faster extension of $S C R$ during turning-off.

$$
\begin{aligned}
v_{e_{-} \text {Plasma }} & =\frac{3}{4} \frac{I_{C E}}{A q N_{\text {Plasma }}} \\
W_{S C R} & =v_{e_{-} \text {Plasma }}\left(t-t_{2}\right)
\end{aligned}
$$

The space charge, $Q_{S C R}$ in Eq.(2.8) and the maximum field strength, $E_{\max }$ 
in Eq.(2.10), increases first linearly with $W_{S C R}$. Accordingly, the collectoremitter voltage $V_{C E}$ increases proportionally to the square of $W_{S C R}$. In the analysis of high-frequency oscillations, the instantaneous chip capacitor of $S C R$ and it's effect of the resonant circuit are important factors. A differential capacitance $C_{\text {diff }}$ can be defined as Eq.(2.9). $C_{\text {diff }}$ acts as the instantaneous chip capacitor. It is inversely proportional to $W_{S C R}$. This can also be applied to the reverse-biased diodes. Generally, larger $C_{d i f f}$ leads to lower resonant frequency in the parasitic resonant circuit.

$$
\begin{array}{r}
Q_{S C R}=q A N_{h_{-}{ }_{n e t} W_{S C R}} \\
C_{d i f f}=\frac{\frac{d Q_{S C R}}{d t}}{\frac{d V_{C E}}{d t}}=\frac{A \epsilon \epsilon_{0}}{W_{S C R}}
\end{array}
$$

Under higher current, $E_{\max }$ raises to the critical field strength $E_{C}$ in short time after the MOS-channel turn-off. $E_{C}$ is about $2 \times 10^{5} \mathrm{~V} / \mathrm{cm}$ for Silicon. The gate resistor limits the discharge current of the gate circuit and thereby slows down the constriction of the MOS-channel. For the building-up of $S C R$ and ramping-up of $V_{C E}$, the larger $R_{g}$ acts in the same direction as a smaller $I_{C E}$ does.

$$
\begin{aligned}
V_{C E} & =\frac{1}{2} \frac{q N_{h_{-} n e t}}{\epsilon \epsilon_{0}} W_{S C R}^{2} \\
E_{\text {max }} & =\frac{q N_{h \_n e t}}{\epsilon \epsilon_{0}} W_{S C R} \\
& =\sqrt{\frac{2 q}{\epsilon \epsilon_{0}} V_{C E} N_{h \_n e t}}
\end{aligned}
$$

With a proportionality $\lambda$ between the nett hole density and the collector current as defined in Eq.(2.12), Eq.(2.11) can be eliminated to Eq.(2.13) 
which makes the relationship between the $E_{\max }$ in the SCR and the $V_{C E}$ as well as $I_{C E}$ visible. For the ideal abrupt channel turn-off $\lambda$ can be taken as $\frac{1}{A q v_{n_{-}} \text {Plasma }}$.

$$
\begin{aligned}
N_{h_{-} \text {net }} & =\lambda I_{C E} \\
E_{\max } & =\sqrt{\frac{2 \lambda q}{\epsilon \epsilon_{0}} V_{C E} I_{C E}}
\end{aligned}
$$

During the forward conduction of IGBT, the anti-parallel diode is of reverse bias, so that during the raise of $V_{C E}$, no plasma in the diode need to be extracted. The space charge density in the diode is then equal to the doping concentration $N_{D}$ in the base region. The $W_{S C R}$ in diode can be calculated according to Eq.(2.14) to estimate the $C_{\text {diff }}$ of diode.

$$
W_{S C R}=\sqrt{\frac{2 \epsilon \epsilon_{0} V_{C E}}{q N_{D}}}
$$

In the IGBT, during the high current turn-off, the $E_{\max }$ reaches the critical value $E_{C}$ at a $V_{C E}$ which is significant below the blocking capability of the IGBT due to the high hole-density in $S C R$. The further increase of the E-field is then limited by the impact ionization. The generated holes are evacuated over $J_{1}$. The electrons drift through the $S C R$ to $J_{2}$. This leads to a reduction of the net hole density and $d E / d x$ in $S C R$. From $t_{3}$, the resulted slew rate reduction on $V_{C E}$ can be observed in the turn-off diagram. After $V_{C E}$ exceeds $V_{D C}$ at $t_{4}$ the load current begins to commutate to the free-wheeling diode. The $I_{C E}$ reduction leads to the further $d E / d x$ reduction in $S C R$ and the reduction of the $S C R$ extension velocity. However, this process is limited by the parasitic inductance in the load current circuit. Up to $t_{5}$ the current has dropped so far that effect of the $d E / d x$ reduction compensates the effect of $S C R$ extension. $V_{C E}$ reaches its maximum at $t_{5}$. After $t_{5}$, the slew rate of $V_{C E}$ can not 
remain positive. From $t_{5}$ to $t_{6} V_{C E}$ decreases rapidly to $V_{D C}$. Up to $t_{7}$, the residual plasma is evacuated by the so-called tail current. Just by $t_{7}$, $S C R$ reaches the field stop layer. The tail current gets rapidly to zero and causes a second peak on the $V_{C E}$.

\subsection{Possible RF-oscillations during IGBT switching}

In the turn-off process of an IGBT, there is always temporary states in which a $S C R$ is already built up and at least part of the load current is still flowing. Because of the high field strength in $S C R$, the charge carriers are accelerated up to the saturation velocities. In case of a superimposed $\mathrm{RF}$-voltage, the charge carrier transit time and the charge injection time would induce a phase shift between current and voltage on the device terminal. At certain frequencies, such phase shifts are between $\pi / 2$ und $3 \pi / 2$. At such frequencies, the power consumptions on the device are negative. The device works therefore as an amplifier. In Chapter 3, the mechanisms and the efficiency of the transit time effect are discussed in detail.

As mentioned previously in 2.2 , the module constructions are always accompanied with parasitic resonant circuits. The noise signal can be raised to disturbing HF-oscillations, if the eigenfrequencies of the resonant circuit match the frequency band of the transit time effect.

\subsubsection{Tail oscillation PETT}

One type of high-frequency oscillation in IGBT modules is reported as PETT(plasma extraction transit time) oscillation in 2002 [GMS02]. Since the oscillation is usually observed in the tail-current phase, it is also referred as tail oscillation. Fig.2.8 shows the diagrams of the PETT oscilla- 


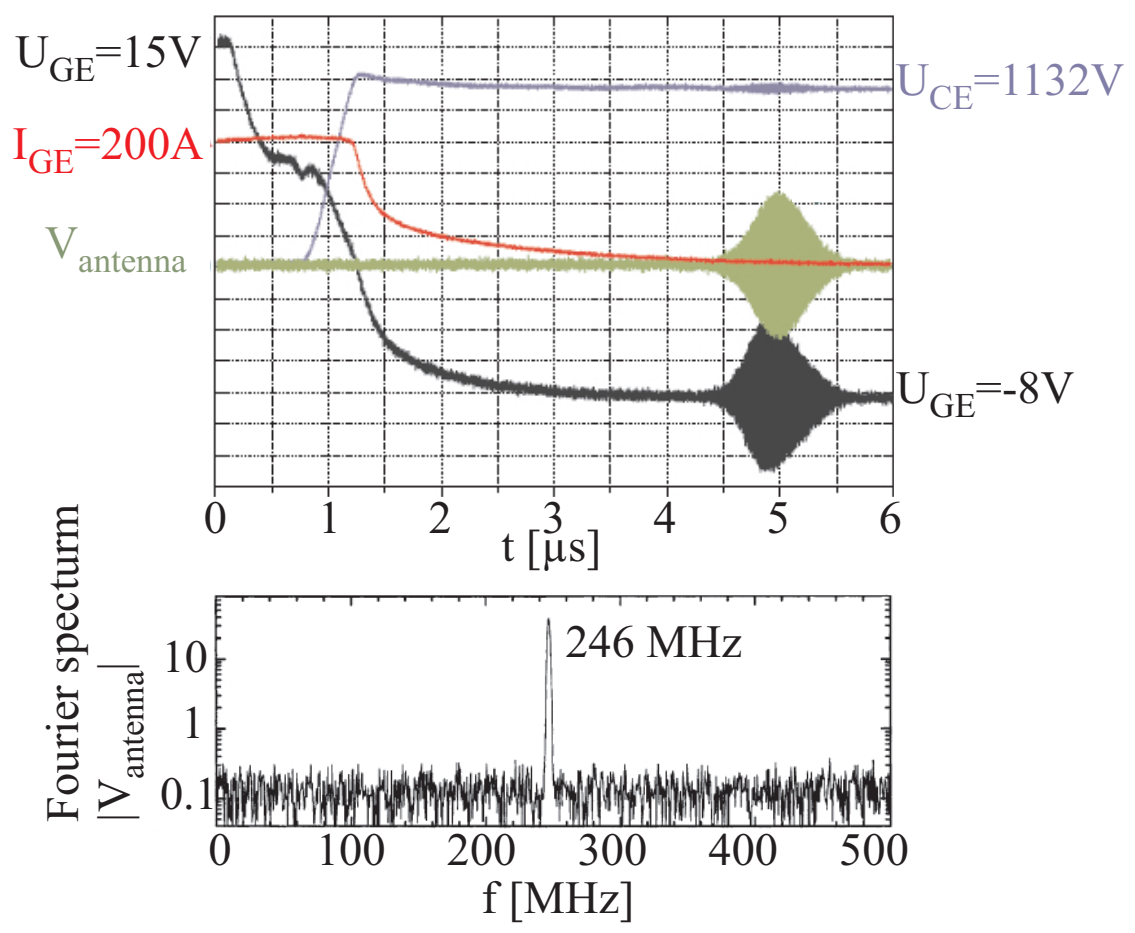

Figure 2.8: PETT oscillation of IGBT [Gut06] 
tion. The oscillation signal is sensed by a wire loop antenna through the electromagnetic coupling. The Fourier spectrum in Fig. 2.8 shows that the frequency of oscillation is around $246 \mathrm{MHz}$. It lies well above the oscillations frequencies caused by negative gate capacitances( 0.4 to $2.5 \mathrm{MHz}$ is shown in [OFO99]). The mechanisms of PETT oscillation was explained to be similar to the mechanisms of a BARITT(Barrier Injection Transit Time) diode. The work mechanism of PETT can be seen in Fig.2.9. The superposition of a small AC-signal on the collector-emitter voltage will lead to a pulse-like holes extraction on the plasma boundary to $S C R$. The AC-signal would be amplified by the transit-time effect periodically, when the semiconductor chip and the module structures build up the appropriate resonant circuits. Finally, the oscillation signal can be perceived as the HF-disturbance. The PETT mechanisms was also found in the turn-off process of fast recovery diodes [SMNL06].

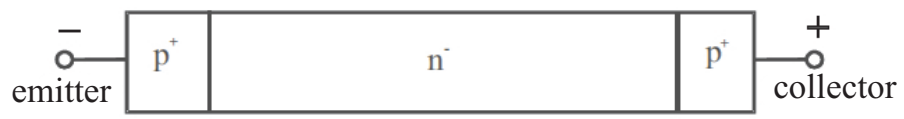

(a)

(b)

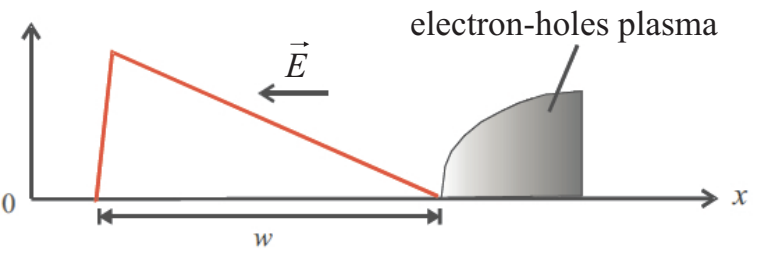

(c)

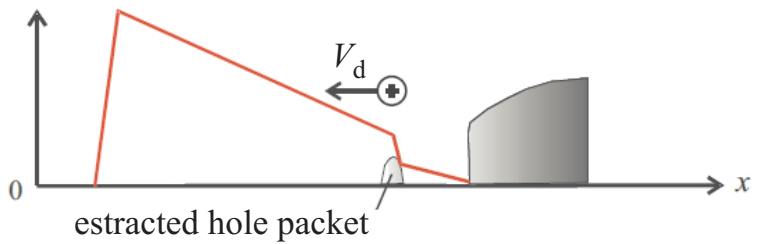

Figure 2.9: Mechanisms of PETT oscillation [Gut06] 
There are generally two methods to suppress oscillations. The first one is to integrate power consumers into the resonant circuit. The power consumer may be resistance in the line, which affects all frequencies. It may also be the resistive coating on the conductor-line and is especially effective for high-frequency signals. Energy of the HF-signal can be extracted also by a resistive conductor adjacent to the resonant circuit. The HFpower is converted to heat by eddy current in such conductor [SGMM03] [SGMM02]. The second method of the oscillation suppression is to mismatch the eigenfrequencies between the amplifier and the resonant circuits.

The emitter cross wire-bonding between parallel IGBTs has already been patented in 1995 as a countermeasure to HF-oscillations during IGBT turn-off as shown here in Fig.2.10. During switching off of modules with paralleled IGBT chips $(3,4)$, high frequency oscillation can cause the electromagnetic interference and the destruction of the chips can still not be excluded. These oscillations can be prevented by the direct emitter wirebonding (14) between the adjacent chips. The cross wire-bondings reduce the inductance between the adjacent IGBTs and thus the resonant circuits are out of tune. There are also proposed solutions by increasing the inductance of the resonant circuit to suppress PETT-oscillation [TKY ${ }^{+}$98]. This usually means the increasing of inductance in the commutation path of the load current. Such inductance is generally unfavourable for highperformance power modules. Additional parasitic capacitances and inductance can also be integrated on the DCB-layout to mismatch the eigenfrequencies [Mou01a]. Countermeasures to PETT-oscillation are summarized in chapter 5 of [Gut06].

Although the PETT-oscillation in the tail current phase is a dynamic process, it shows certain quasi-stationary characteristics. The IGBT has taken over the entire DC-link voltage before PETT-oscillation appears. The collector-emitter voltage remains almost unchanged during PETToscillation. The $S C R$ capacitance of IGBT- and diode-chip as well as the 


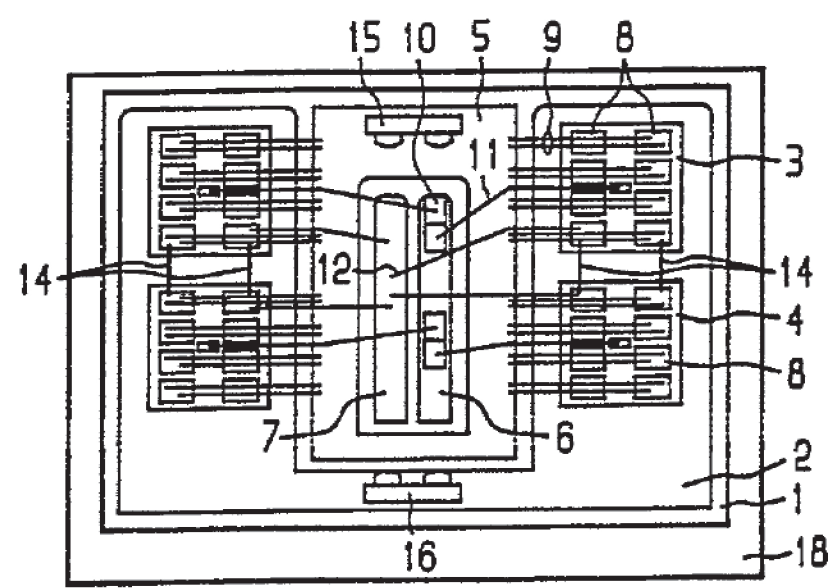

Figure 2.10: Cross wire-bonding to suppress the high-frequency turn-off oscillation in IGBT modules - EUPEC patent Zimmermann 1995 [ZS95]

eigenfrequencies of the resonance circuits in the module-structure vary only within an narrow range. For example, tail oscillations were found in the range between $74 \mathrm{MHz}$ and $78.2 \mathrm{MHz}$ on a $4500 \mathrm{~V}$ IGBT module [FHK09]. Therefore, the mismatching between the resonant circuit and the transit time effect can be relatively simply realized.

\subsubsection{Dynamic Impact-Ionization Transit-Time oscillations of freewheeling diodes}

It is already known that avalanche generation my accrue in the reverse recovery phase even for a reverse bias voltage below the steady-state breakdown voltage [BS67]. This phenomenon is similar to the dynamic avalanche during the turning off of GTO-thyristors and IGBTs [SN89] and of the fast recovery Si diodes [DLS03]. High-frequency oscillations have been found during the turning-off of fast recovery diode in the dynamic avalanche phase [LSG98] [Lut06]. Fig.2.11 shows the circuit of the measurement 
setup to test the reverse recovery behaviour of the freewheeling diode in the IGBT Module by double pulse mode. The top side IGBT of the half bridge is actively switched with double pulse during the bottom IGBT is kept turned-off. The load current in $L_{p}$ is adjusted by the $V_{D C}$ and the first pulse width. After the first pulse, the top IGBT is in off-state and the bottom freewheeling diode (DUT) is loaded with the current in $L_{P}$. As the top IGBT is turned-on again, the reverse recovery characteristic of the freewheeling diode occurs. The dynamic oscillation is observed after the recovery current peak and during the tail current phase of the diode.

Fig.2.12 shows the measurement on the $1200 \mathrm{~V}$ diode at $0^{\circ} \mathrm{C}$ [LSG98]. After the Reverse recovery current peak (time point $t_{1}$ ) the reverse current decreases. Between $t_{1}$ and $t_{2}$, the voltage climbs up close to the value of the DC-link voltage, while the tail current flows in the diode. After $t_{2}$ a current hump grows from the tail current. This hump occurs only if the DC-link voltage is above $910 \mathrm{~V}$. The further increase in the DC-link voltage to $930 \mathrm{~V}$ effects that a high reverse current suddenly shoots up from the current hump whose amplitude is a multiple of the reverse recovery current peak. A high frequency oscillation in the range $>500 \mathrm{MHz}$ is superimposed. After some 100ns $\left(t_{4}\right)$, the oscillation is finished. A reduction of the voltage by only 1 or $2 \mathrm{~V}$, or an increase of the temperature by 1 or $2^{\circ} \mathrm{C}$, removes the effect [LSG98].

The dynamic IMPATT oscillation is caused by the K-centre, which is created at irradiation of the semiconductor with high-energy particles [LSG98] for example in the Controlled Axial Liefetime (CAL) technology [LS94] [SL04]. The K-center has the characteristic of a temporary donor and is positively charged during the forward conduction. After the voltage has changed its polarity the K-centre need to be discharged. The time constant of the discharge process is temperature dependent. It is ca. 100ns at $400 \mathrm{~K}$ and is in the order of some microseconds at temperatures below 300K [LSSD11]. Especially for low temperature, when the IGBT applies 
the DC-link voltage to the diode in a very short time after the the current zero-crossing point of the diode, the break down voltage of the diode is temporarily reduced due to the positively charged K-center and impact ionization sets on [LSSD11]. Fig.2.12 shows the measurement on a $1200 \mathrm{~V}$

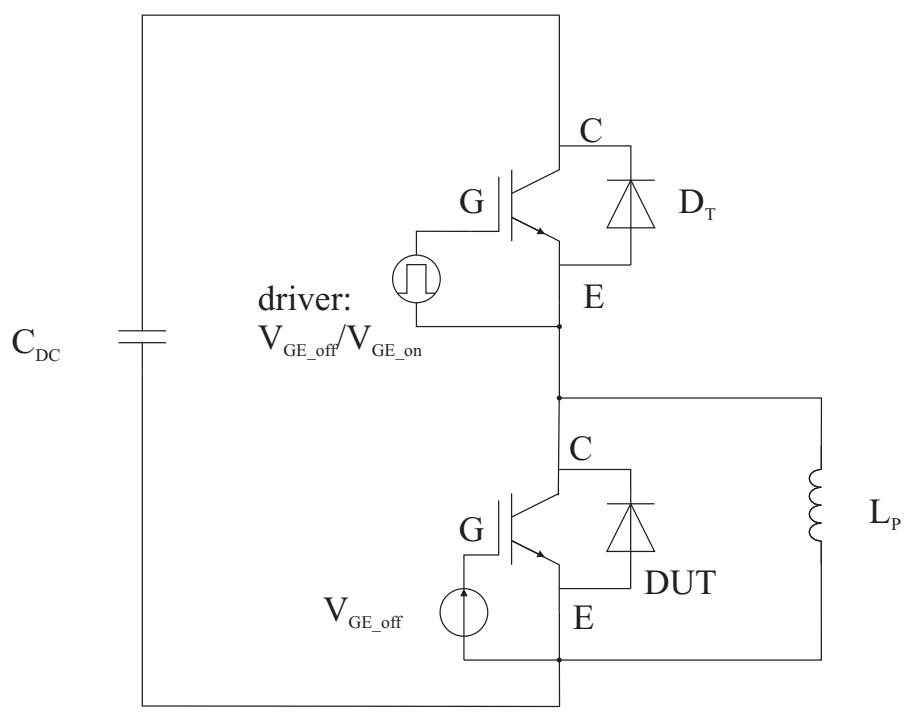

Figure 2.11: Test circuit of the reverse recovery behaviour

diode at $0^{\circ} \mathrm{C}$. The work mechanism is shown in Fig.2.13. The electron packets generated by the avalanche run through the SCR to the right side. During the dynamic avalanche the device has the similar property as the IMPATT diode [Sze81] and is able to raise HF-oscillations. To avoid these oscillations, it is necessary to restrict the number of the generated donor states. The irradiation parameter as well as annealing temperature and time have to be chosen carefully [SSL02b] [SLH04]. Early works [SSL02a] [SL04] show the possibility to optimize the irradiated devices by means of simulations. The dynamic IMPATT oscillation can be surely avoided by the the proper adjustment of the K-centre concentration [LSSD11] 


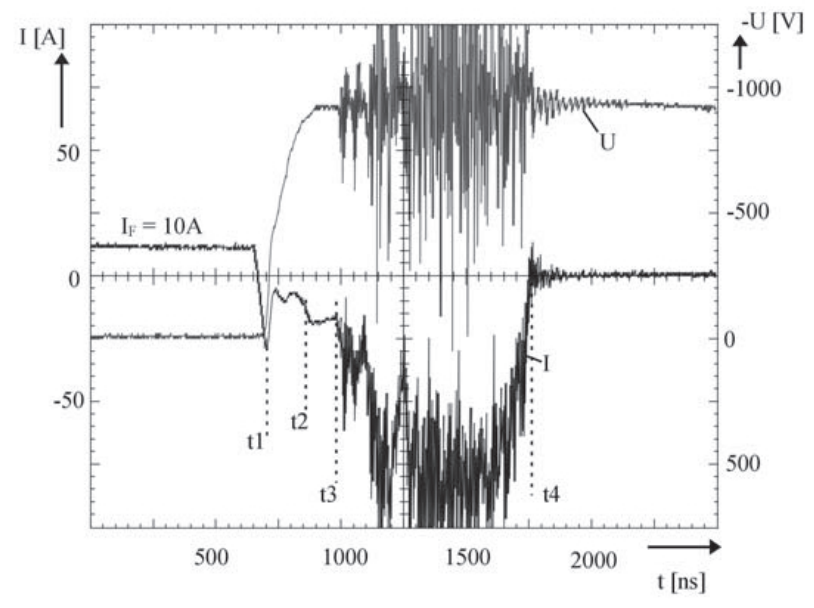

Figure 2.12: Dynamic IMPATT oscillation measured at $0^{\circ} \mathrm{C}$ on $1200 \mathrm{~V}$ diode [LSG98]

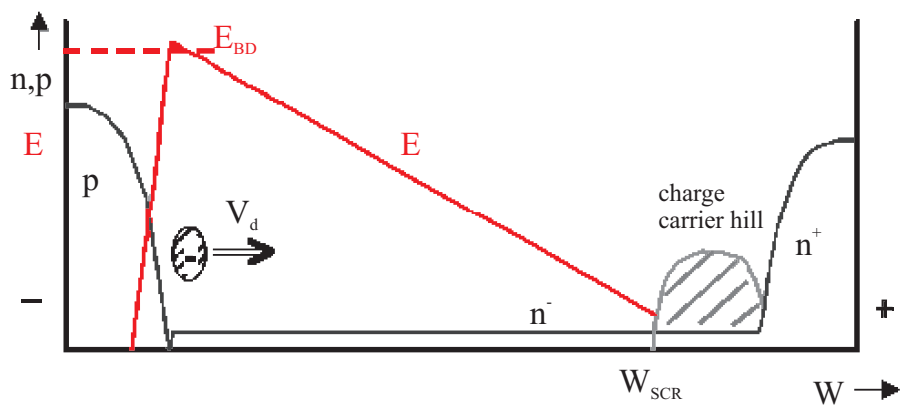

Figure 2.13: Process in the device at dynamic IMPATT oscillation [LSSD11] 


\subsubsection{Transient Avalanche oscillations}

Meanwhile, a new type of HF-oscillation was found during the IGBT high current turn-off. Fig.2.14 shows TA-oscillation [HPB $\left.{ }^{+} 14\right]$ during high current turn-off of $3.3 \mathrm{kV}$ IGBT. The oscillation appears only during the rise of $V_{C E}$ and fall of the $I_{C E}$ for several hundreds $n s$. And it was observed only during the dynamic avalanche process. TA-oscillations occur during the fast expanding the SCR. Therefore, in contrast to the PETT-oscillation, TA-oscillation shows even more dynamic characteristics. During the TAoscillation, high current is still flowing through the IGBT. The typical frequency of TA-oscillations is found between $100 \mathrm{MHZ}$ and $700 \mathrm{MHz}$. This indicates the relevance of TA-oscillation to the transit time effect. The understanding of the mechanism of TA-oscillation by analysis of measurements and simulations is presented in Chap.4.

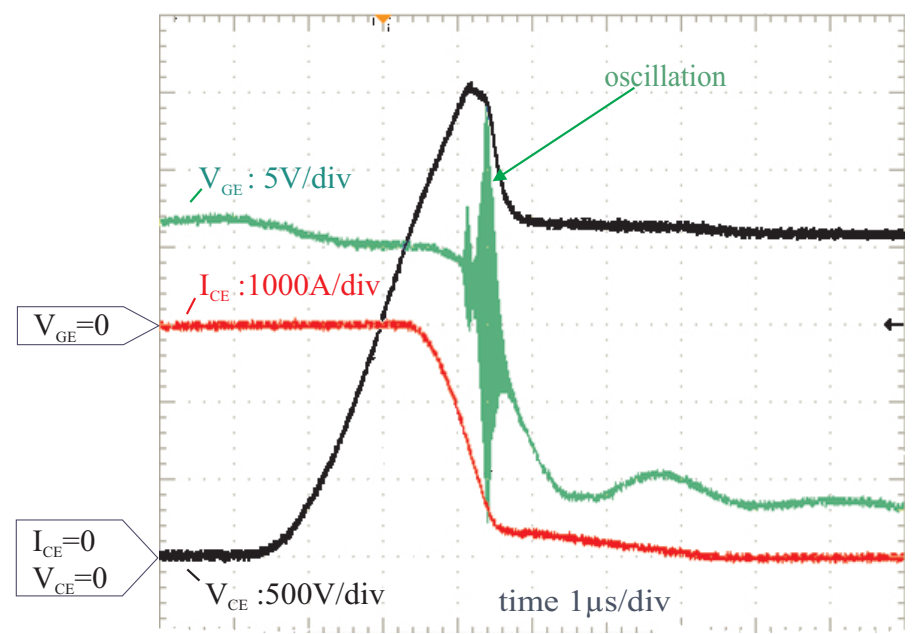

Figure 2.14: Measurement diagram of TA-oscillation, adapted from $\left[\mathrm{HPB}^{+} 14\right]$ 


\section{Chapter 3}

\section{Mechanisms of Transit time effect and RF-oscillations in IGBT Modules}

When a point charge is moving in a conductor system, the terminal current of the conductor system is determined by the movement of the point charge but not the charge carriers arriving to the electrodes [Sho38]. This is known as "Ramo-Shockley theorem". Using this theorem the transit time effect of charge carriers through the space charge region can be explained. IMPATT diodes and BARITT diodes are the devices based on the similar principle corresponding to the transit time effect. In the diodes, a space charge region (SCR) is formed at a reverse biased pn-junction by a DCvoltage. The charge injection into the $S C R$ can be induced by a AC voltage supplied additionally to the DC voltage. In a IMPATT diode, the $E_{\max }$ is set to just below the $E_{C}$ so that the additional AC-voltage would drive the $E_{\max }$ to exceed $E_{C}$. The charge injection is caused by the avalanche generation. In the BARITT diodes, there is a forward biased pn-junction 


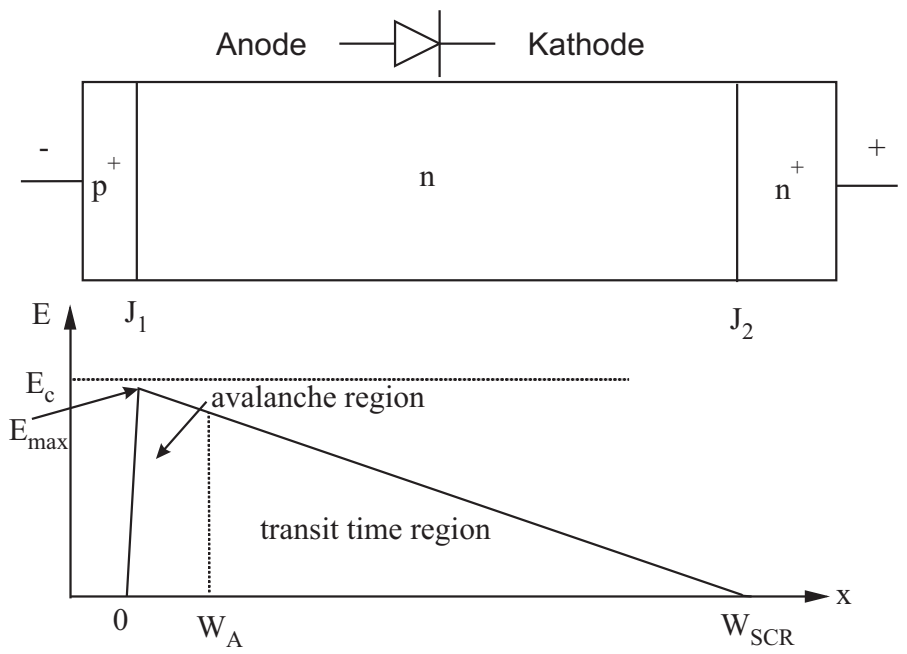

Figure 3.1: Structure and work mechanisms of IMPATT diode

opposite to the blocking pn-junction $J_{2}$. Under he DC-voltage, the $J_{2}$ stands just below the threshold voltage. The additional AC-voltage to the DC-voltage would drive the $J_{2}$ into the forward conduction. The forward current of $J_{2}$ induces the charge injection into the $S C R$. The injection current needs a certain injection time $T_{i n j}$ to reach its maximum before it vanishes in the negative half period of the AC-voltage. The injected charge carriers need a transit time $T_{D}$ to drift through the $S C R . T_{i n j}$ and $T_{D}$ together induce a phase shift between the AC terminal voltage and the $\mathrm{AC}$ terminal current of the diodes. In a certain frequency range, in which the phase shift is between $1 \pi / 2$ and $3 \pi / 2$, the diode acts as amplifier.

\subsection{Amplification process}

The IMPATT diode is an avalanche transit time structure. The simplest variant of the avalanche transit time structure is shown in Fig. 3.1. Under the reverse bias voltage $V_{D C}$, the $S C R$ is built up around $p^{+} n$ junction 
$J_{1}$. On the $p^{+}$side, the E-field decreases steeply to zero. Due to the weak doping level of the $\mathrm{n}$ region, the $S C R$ stretches wide into it. At the operation point, the $V_{D C}$ is adjusted just below the breakdown voltage. The maximum field strength $E_{\text {max }}$ directly at the position $J_{1}$ is just below the critical field strength $E_{c}$. In operation, an AC voltage $V_{A C}$ is superimposed on $V_{D C}$. In the positive half cycle of $V_{A C}$, the structure is driven into avalanche. Electron-hole pairs are generated near $J_{1}$. The holes are extracted through $p^{+}$to anode during the electrons have to drift through the transit-time region to the cathode side. The E-field in the drift region is adjusted high enough so that the drift velocity of the charge carriers reach the saturation velocities $v_{s}$. As already described in Chap.2, a similar avalanche transit-time structure is formed from $t_{3}$ (Abb. 2.6) during turn-off of the IGBT.

\subsubsection{Avalanche breakdown}

Under the strong E-field, electrons / holes can be so strongly accelerated that they gather sufficient energy on their free-moving length to generate new electron-hole pairs through the collision with the next atomic lattice. The ionization coefficient $\alpha_{n, p}$ indicates the number of electron-hole pairs generated by an electron / hole on the distance of $1 \mathrm{~cm}$. The structure comes into breakdown, when the charge carrier multiplication in the $S C R$ leads to a infinity current. The avalanche breakdown criterion is given by equation (3.1) in [Sze81] S.100.

$$
\int_{0}^{W} \alpha_{n} \exp \left(-\int_{x}^{W}\left(\alpha_{n}-\alpha_{p}\right) d x^{\prime}\right) d x=1
$$

The equation (3.1) can be simplified to (3.3) using the definition of the effective ionization coefficient $\alpha_{\text {eff }}$ in equation (3.2) [LSSD11] P.62. For the E-field strength over $1 \times 10^{5}$, the ionization rate increases exponentially. Fig. 3.2(a) shows the field dependence of the ionization coefficient 
(a)

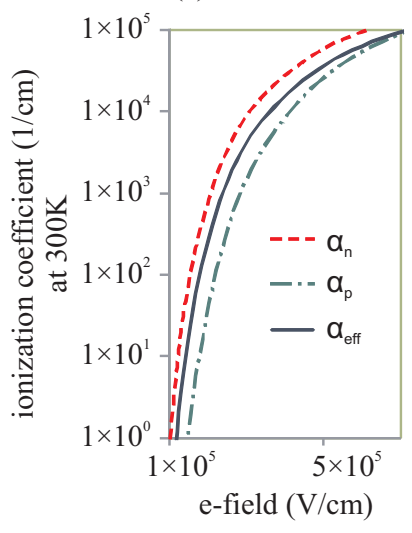

(b)

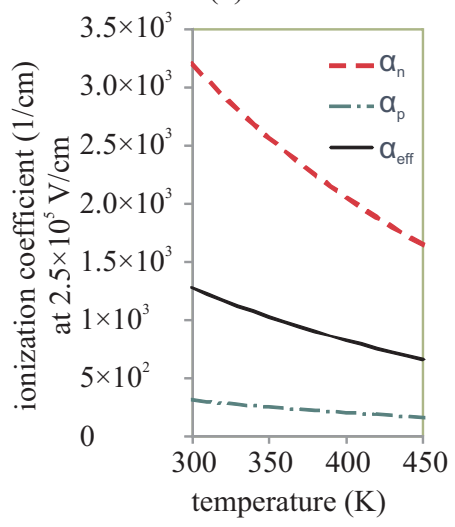

Figure 3.2: (a) The ionization coefficient as a function of E-field at 300K, similar to Fig. in [LSSD11] P.65. (b) The temperature dependence of the ionization coefficient under the E-field of $2.5 \times 10^{5} \mathrm{~V} / \mathrm{cm}$

for silicon at 300K [LSSD11].

With increasing temperature, the free-moving length and thus the ionization coefficient decreases. The temperature dependence of the ionization coefficient between $-20^{\circ} \mathrm{C}$ and $150^{\circ} \mathrm{C}$ for silicon can be described by equations (3.4) to (3.6) with good approximation [LSSD11] P.64. Fig. 3.2(b) shows the temperature dependence of the ionization coefficients for silicon under the E-field strength of $2.5 \times 10^{5} \mathrm{~V} / \mathrm{cm}$. From $300 \mathrm{~K}$ to $450 \mathrm{~K}$ the ionization coefficients decrease by roughly $50 \%$.

$$
\begin{aligned}
& \alpha_{e f f}=\frac{\alpha_{n}-\alpha_{p}}{\ln \left(\alpha_{n} / \alpha_{p}\right)} \text { [LSSD11] P.61. } \\
& \int \alpha_{e f f} d x=1
\end{aligned}
$$




$$
\begin{gathered}
\alpha_{n}=1.1 \times 10^{6} \cdot \exp \left(-\frac{1.46 \times 10^{6}+1100(T-300 K)}{E}\right) \mathrm{cm}^{-1} \\
\alpha_{p}=2.1 \times 10^{6} \cdot \exp \left(-\frac{2.2 \times 10^{6}+1100(T-300 K)}{E}\right) \mathrm{cm}^{-1} \\
\alpha_{e f f} \cong 1.06 \times 10^{6} \cdot \exp \left(-\frac{1.68 \times 10^{6}+1100(T-300 K)}{E}\right) \mathrm{cm}^{-1}
\end{gathered}
$$

The avalanche region is generally defined as eq. (3.7), where the integral of the ionisation coefficient along the avalanche region reach 0.95 [Sze81] P.573.

$$
\int_{0}^{W_{A}} \alpha_{e f f} d x=0.95
$$

\subsubsection{Amplification efficiency}

Fig. 3.3 shows the diagram of terminal voltage, charge injection and the induced current of the transit time structure. The terminal voltage is a result of the AC-voltage override $V_{R F} \sin (\omega t)$ to the $V_{D C}$.

$$
V_{T}=V_{D C}+V_{R F} \sin \omega t
$$

According to the "'Ramo-Shockley theorem"', the diode current is equal to the average convection current through out the drift region ([Pau81] P.315). When a charge packet of $Q$ is injected into the $S C R$ at the position of $W_{A}$ and drifts with the velocity of $v_{Q}$, as shown in Fig.3.1, the inducing current $J_{\text {ind }}$ on the terminal can be described by Eq.3.9 [EH98] P.354.

$$
J_{i n d}=\frac{Q}{W_{S C R}}\left(v_{Q}-\frac{W_{A}}{W_{S C R}} \frac{d W_{S C R}}{d t}\right)
$$



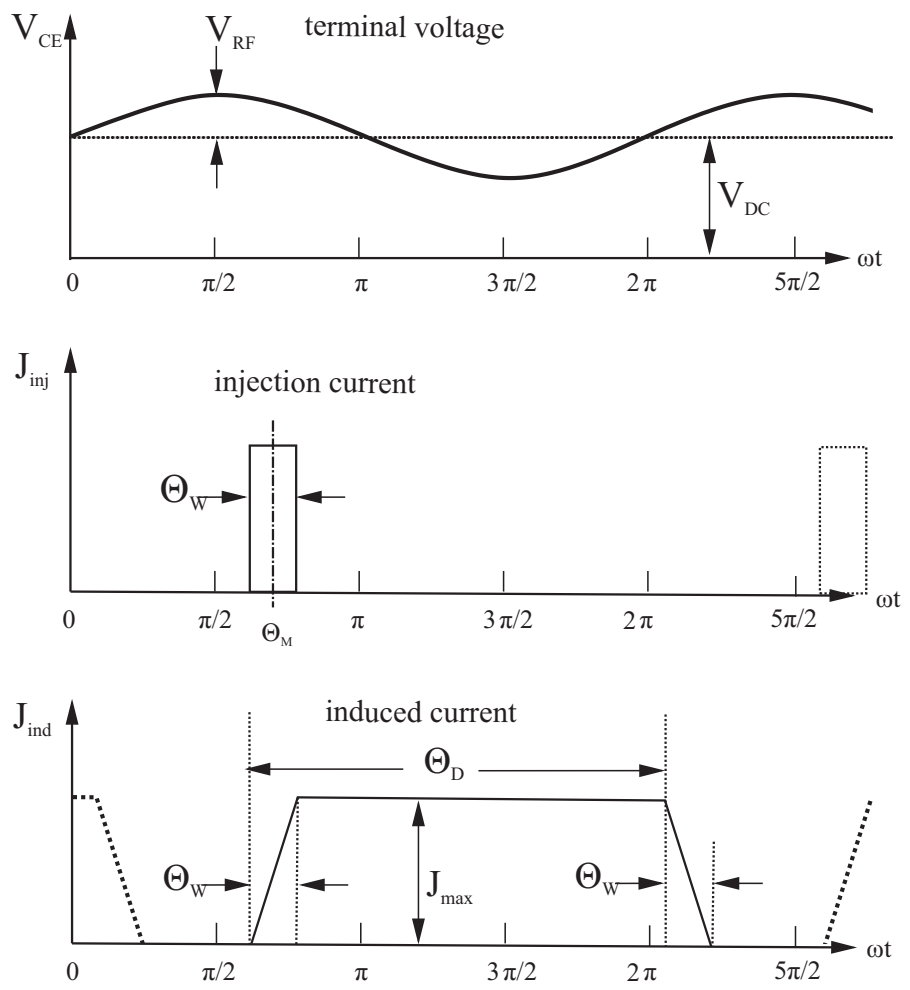

Figure 3.3: Diagram of voltage and current in a transit time structure with the simplified charge injection, Fig. from [EH98], extended

In the positive half period of $V_{R F}$, electrons are injected into the drift region in the injection time $T_{i}$ with a injection angle $\Theta_{W}\left(=T_{i} \times \omega\right)$. The injected electrons need a transit time of $T_{D}\left(=W / v_{s}\right)$ to drift through the SCR. The transit angle is calculated as $\Theta_{D}=T_{D} \times \omega$. Assume that a charge pulse is injected into the drift region at the phase angle $\Theta_{M}$. The diagram of $V_{T}, V_{R F}$, charge injection and the induced terminal current $J_{\text {ind }}$ are shown in Fig.3.3 (generalization on the base figure on [EH98] P.355.). The ability to generate RF-power by the transit time structure can be described using the efficiency coefficient $\eta$ as in Eq.(3.13). The 
efficiency coefficient is the ratio of the RF-power generation to the total power consumption which is drawed from the DC-voltage source $V_{D C}$. The introduction steps are involved in Appendix A

$$
\begin{aligned}
J_{D C} & =\frac{1}{2 \pi} \int_{\Theta_{M}-\Theta_{W} / 2}^{\Theta_{D}+\Theta_{M}+\Theta_{W} / 2} J_{i n d}(\omega t) d(\omega t) \\
P_{D C} & =J_{D C} V_{D C} \\
P_{R F} & =-\frac{A}{2 \pi} \int_{\Theta_{M}-\Theta_{W} / 2}^{\Theta_{D}+\Theta_{M}+\Theta_{W} / 2} J_{i n d}(\omega t) V_{R F} \sin (\omega t) d(\omega t) \\
\eta & =\frac{P_{R F}}{P_{D C}}=\frac{V_{R F}}{V_{D C}} \frac{\sin \left(\frac{\Theta_{W}}{2}\right)}{\frac{\Theta_{W}}{2}} \frac{\cos \left(\Theta_{M}+\Theta_{D}\right)-\cos \Theta_{M}}{\Theta_{D}}
\end{aligned}
$$

The injection dependent efficiency coefficient $\eta_{1}$ (3.14) and the transit time dependent efficiency coefficient $\eta_{2}(3.15)$ can be eliminated out of $\eta$. $\eta_{1}$ gets its maximum as the $\Theta_{W}$ goes to Zero. The efficient coefficient favoured from the short injection pulse. In an IMPATT diode, the charge injection reach its maximum roughly at $\Theta_{M}=\pi$. For the BARITT diode, the maximal charge injection happens at $\Theta_{M}=\pi / 2$. The optimal width of drift region and the corresponding transit angle can therefore be estimated for the most effective Rf-power conversion. For IMPATT diode, the optimal transit angle $\Theta_{D} \approx 3 \pi / 4$. And for BARITT diode the optimal transit 
angle ist found to be $\Theta_{D} \approx 3 \pi / 2$ [EH98] P.355.

$$
\begin{aligned}
\eta_{1}\left(\Theta_{W}\right) & =\frac{\sin \left(\frac{\Theta_{W}}{2}\right)}{\frac{\Theta_{W}}{2}} \\
\eta_{2}\left(\Theta_{D}\right) & =\frac{\cos \left(\Theta_{M}+\Theta_{D}\right)-\cos \Theta_{M}}{\Theta_{D}}
\end{aligned}
$$

As Eq.(3.13) is derived without the interval limitation of $2 \pi$, it can be applied to the case of wide drift regions as in IGBT $\left(\Theta_{M}+\Theta_{D} \geq 2 \pi\right)$. In the frequency range that the transit angle lies in $(2 n-1) \pi<\Theta_{D}(2 n-1) \pi$, the BARITT and the corresponding PETT mechanisms have the ability to generate RF-power. The IMPATT mechanism can theoretically generate Rf-power in the whole frequency range. As can be seen in Fig.3.4, $\eta_{2}$ has at least a second and a third peak value in addition to its maximum. The peak value of $\eta_{2}$ reduces with the increase of the transit angle, but the second peak value of IMPATT mechanism is still as high as the maximum of BARITT structure. The PETT and BARITT mechanism shows the damping effect as long as the the frequencies are low [Sil14]. The drift velocity of electrons and holes $\left(v_{d n}, v_{d p}\right)$ can be derived on the basis of the measurement of electron and hole drift velocity in Silicon [CMMO75]. The equations Eq.(3.16)and Eq.(3.17) describe the dependencies on temperature and E-field [Bal79] P.10.

$$
\begin{aligned}
& v_{d n}=\frac{1.42 \times 10^{9} T^{-2.42} E}{\left[1+\left(E / 1.01 T^{1.55}\right)^{2.57 \times 10^{-2} T^{0.66}}\right]^{\left(2.57 \times 10^{-2} T^{0.66}\right)^{-1}}} \\
& v_{d p}=\frac{1.31 \times 10^{9} T^{-2.2} E}{\left[1+\left(E / 1.24 T^{1.68}\right)^{0.46 \times 10^{-2} T^{0.17}}\right]^{\left(0.46 \times 10^{-2} T^{0.17}\right)^{-1}}}
\end{aligned}
$$

Under the assumption of a triangle E-field distribution and the maximal E-field of $2 \times 10^{5} \mathrm{~V} / \mathrm{cm}$, the effective velocities of electrons and holes $\left(\bar{v}_{d n}\right.$, 


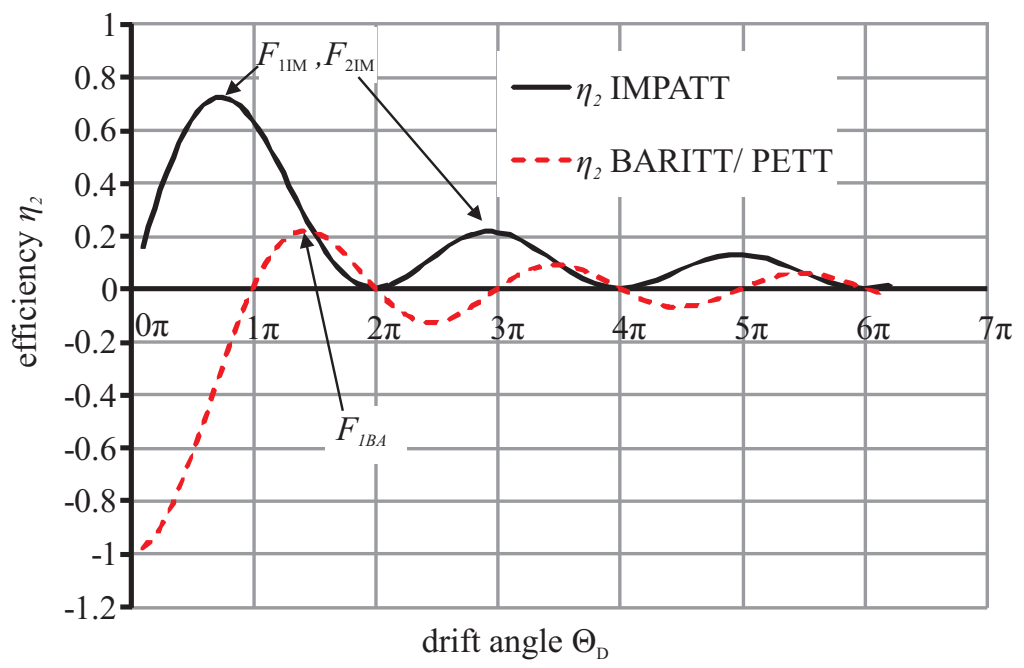

Figure 3.4: $\eta_{2}$ of IMPATT und BARITT/PETT structures according to the transit angle

$\left.\bar{v}_{d p}\right)$ in the SCR are estimated. This results under $25^{\circ} \mathrm{C} \bar{v}_{d n} \approx 8.93 \times$ $10^{6}$ and $\bar{v}_{d p} \approx 6.44 \times 10^{6}$. As shown in Fig.3.5, the eigenfrequencies of the transit structures decrease according to the expansion of the SCR. The frequency $F_{1 B A}$, at which the BARITT structure is most effective, is about $3 / 2$ of $F_{1 I M}$. The IMPATT structure at $F_{2 I M}$ is still as effective as BARITT structure at $F_{1 B A}$. The transit time frequency $F_{2 I M}$ is almost 4 times higher as $F_{1 I M}$. The internal states during the IGBT turning-off are shown in Fig. 4.35. It is generally possible that the IMPATT and PETT mechanisms exist temporally parallel to each other. The SCR acts as the transit region of electrons for the IMPATT mechanism and also as the transit region of holes for the PETT mechanism. The IGBT chips are physically constructed so that they can generally act as RF-generator during the turn-off process. If an adequate resonant circuit exists and it is not well damped, then the thermal noise would be raised to oscillations. 


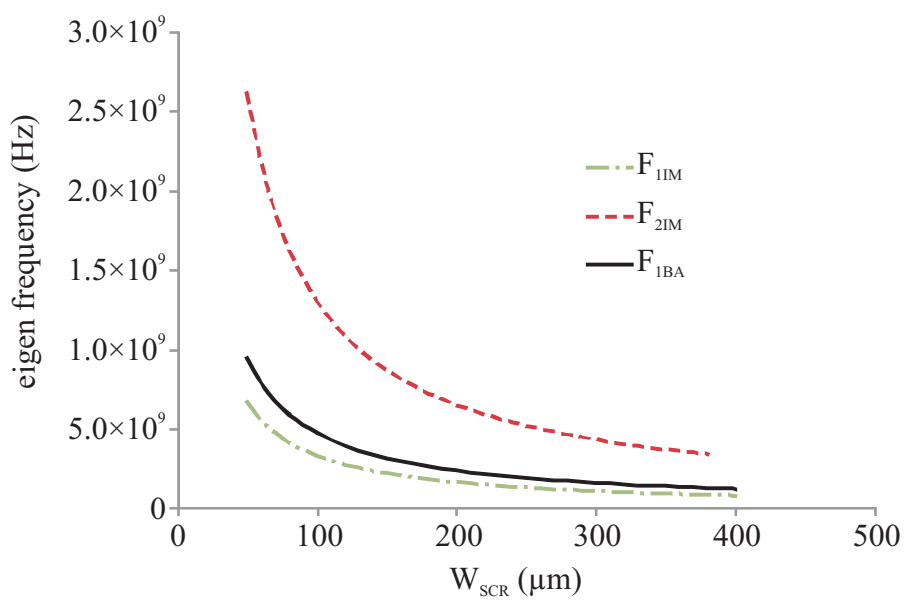

Figure 3.5: Transit angle dependent efficiencies of RF-power Generation $\eta_{2}$ in IMPATT und BARITT/PETT structure using $\bar{v}_{d n} \approx 8.93 \times 10^{6}$ and $\bar{v}_{d p} \approx 6.44 \times 10^{6}$

\subsection{Resonant circuits in IGBT modules}

There are always rich number of resonant circuits in the IGBT applications. They may be constructed of parasitics of the module setups, diode chips and even IGBT chip itself. For the analysis of RF-oscillation, the resonant circuit near to the IGBT chips are of special interest. Fig.2.3 shows for example that the parasitic capacitance between DCB areas and the parasitic inductance can be found near the IGBT- and diode chips. The three resonant circuits in this construction are shown in Fig.4.19. The chip capacitance for the RF-analysis can be estimated according to Eq.(2.9). As the chip capacitances are inversely proportional to the width of SCR, the eigenfrequencies of the resonant circuits increase with the expanding of SCR. At the moment that the eigen frequency matchs the transit time frequencies as shown in Fig.3.5, RF-oscillations can be raised. The quantitative analysis are presented according to the sample setups in Chap.4. 


\section{Chapter 4}

\section{Analysis of Transient}

\section{Avalanche oscillation}

Under high current density, high frequency oscillation signals are found on the trace of gate-emitter voltage during the turning off of $3.3 \mathrm{kV}$ IGBTs as shown in Fig. 2.14. This leads to the presumption that gate capacitance and the Miller-feedback could be responsible for the this oscillation. However this presumption is disproved by the measurements and simulations introduced in this chapter. Moreover the RF-signals are found to be emitted by the emitter wire bonds. As this oscillation occurs only transiently during the dynamic avalanche of the IGBT, it is called TAoscillation in this work. The resonant circuits of TA-oscillations can be localized nearby the IGBT chips. The parasitics extraction of setups on DCB and the analytical estimations of IGBT and diode chip-capacitance allow the quantitative analysis of the resonant circuits according to the eigenfrequencies. The mechanisms of TA-oscillation are illustrated corresponding to the transit time effect and are verified by device simulations. 


\subsection{Measurement of TA-oscillations}

Systematic measurements are accomplished on $3.3 \mathrm{kV}$ IGBT modules and substrates setups to find out the source and the relevant parameters of TA-oscillations.

\subsubsection{Measurement of TA-oscillation on IGBT mod- ules}

TA-oscillations are first observed on an $3.3 \mathrm{kV}$ IGBT module under development, in which $24 \times$ IGBT chips are parallel arranged and act as a single switch. Two modules are series connected to a half-bridge for the dynamic characterization as shown in Fig.2.4. The hardware configuration of the dynamic characterization is represented in Fig.4.1. Because of the high voltage, the dynamic characterizations are generally carried out in a grounded security box. For the conventional measurements three channels of oscilloscope are necessary to record $V_{C E}, V_{G E}$ and $I_{C E}$. A $1000 \times$ voltage probe is applied for $V_{C E}$. A $10 \times$ voltage probe is applied for $V_{G E}$. $I_{C E}$ is converted to voltage signal using a Pearson current transformer. Additionally to the conventional dynamic module characterization, for the oscillation analysis, a wire loop is placed near the DUT to capture the RFdisturbance. Care must be taken for the parasitics in the ground loop in order to distinguish the real RF-signal and the interferences, see appendix B.

Similarly to the PETT oscillation, the oscillation frequencies of TAoscillation are in the range of several hundreds of $\mathrm{MHz}$. But unlike the PETT oscillation, TA-oscillation does not appear in the phase of the tail current. TA-oscillations are found during the dynamic avalanche of the high current turn-off of the IGBT. As TA-oscillation happens, the DUT is under high voltage and high current density. 


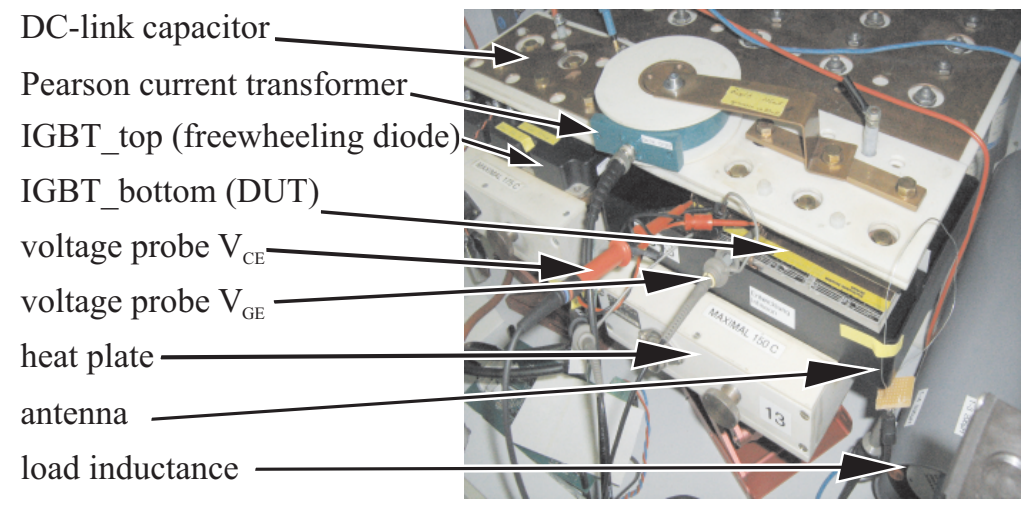

Figure 4.1: Test setup for the dynamic characterization of IGBT modules

\section{Effect of load current $I_{L}$ and DC-link voltage $V_{D C}$}

Three measurement series, MS1, MS2 and MS3, are processed to verify dependences on $I_{L}$ and $V_{D C}$. The DUT and roadmap of the measurements is shown in Fig. 4.2. In MS1, the $V_{D C}$ is kept at $2100 \mathrm{~V}$ during the varying of $I_{L}$ by $T_{o n}$. In MS2, the $I_{L}$ is kept at $3200 \mathrm{~A}$ during the varying of $V_{D C}$ and $T_{o n}$. In MS3, the $L_{P}$ and $T_{o n}$ are kept constant. According to Eq.(2.2), the variation of $V_{D C}$ leads simultaneously to a proportional change of $I_{L}$.

TA-oscillations are found under various combinations of $I_{L}$ and $V_{D C}$. Fourier transform is applied on the measured signals to evaluate the oscillation in the frequency domain. Fig.4.3 shows the discrete Fourier transform (FFT) of the antenna signals measured in MS1. The RF-oscillation of $625 \mathrm{MHz}$ is found under the condition of $V_{D C}=2100 \mathrm{~V}$ and $I_{L}=3200 \mathrm{~A}$, right in Fig.4.3. The amplitude of the oscillation decreases first with the reduction of turn-off current $I_{L}$. The oscillation vanishes until $I_{L}=2800 \mathrm{~A}$ and appears again at $I_{L}=2500 \mathrm{~A}$ with a lower frequency of about $300 \mathrm{MHz}$, left in Fig.4.3. Further reduction of $I_{L}$ leads to the final oscillation-free turn-off. In MS2, $I_{L}$ is kept at $3200 \mathrm{~A}$ during the varying of $V_{D C}$. The 


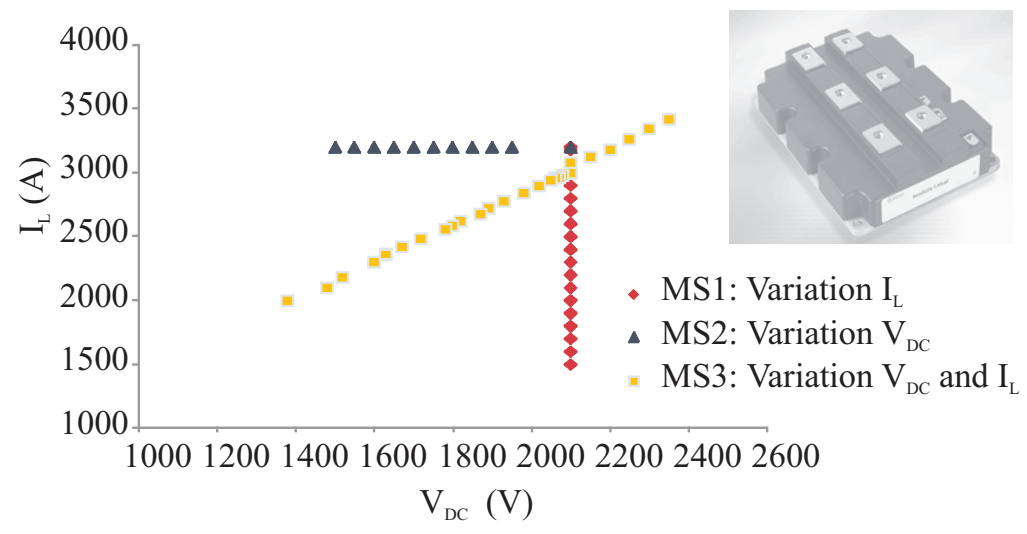

Figure 4.2: Roadmap of measurement series to find out the dependences of TA-oscillations on $I_{L}$ and $V_{D C}$.
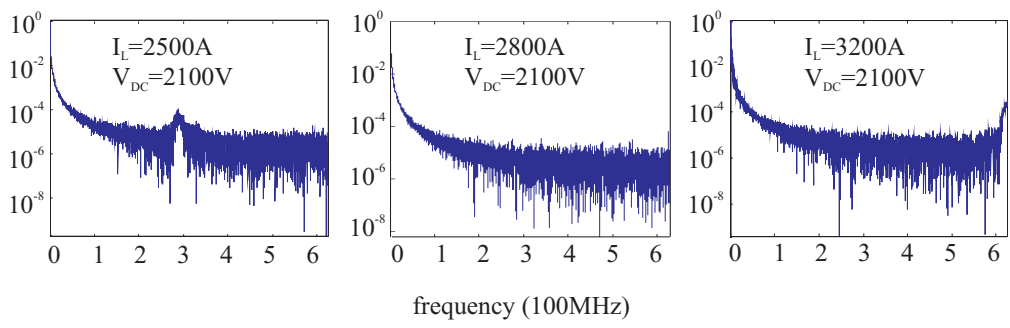

Figure 4.3: Discrete Fourier transform (FFT) of antenna signal of MS1: variation of load current $I_{C E}$ 
varying of DC-link voltage shows the influence on the TA-oscillation in the same manner as the varying of $I_{L}$. As shown in Fig.4.4, after the $625 \mathrm{MHz}$ oscillation is found at $3200 \mathrm{~A} I_{L}$ and $2800 \mathrm{~V} V_{D C}$ the $V_{D C}$ is reduced. The oscillation almost disappears as $V_{D C}$ decreases from $2100 \mathrm{~V}$ to $1800 \mathrm{~V}$ and appears again at $V_{D C}=1650 \mathrm{~V}$ with the lower frequency of about $300 \mathrm{MHz}$. For the DC-link voltage below $1600 \mathrm{~V}$, no oscillation is found during the turn-off process. Fig.4.5 shows that MS3 has same tendency as MS2 and
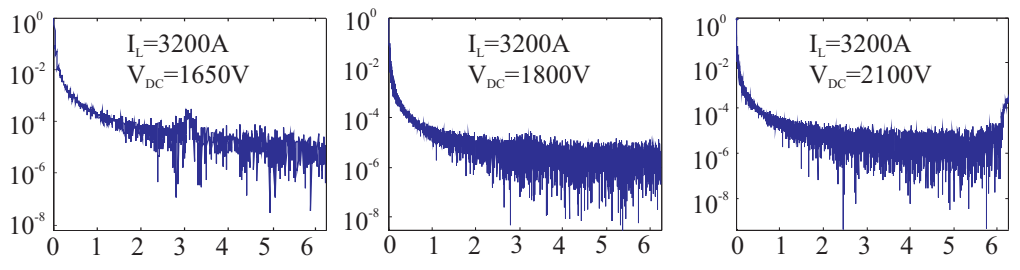

frequency $(100 \mathrm{MHz})$

Figure 4.4: Discrete Fourier transform (FFT) of antenna signal of MS2: variation of DC-link voltage $V_{D C}$

MS1. In the measurement serie MS3, the $300 \mathrm{Mh}$ oscillation is found at $2720 \mathrm{~A} I_{L}$ and $1890 \mathrm{~V} V_{D C}$. The Product of $V_{D C}$ and $I_{L}$ is found to be a key parameter for the TA-oscillations. The frequency of the oscillations under the hard condition $\left(I_{L} \times V_{D C}=6.7 \times 10^{6} \mathrm{VA}\right)$ can identified at about $625 \mathrm{MHz}$, and the frequency of the oscillation under soft conditions
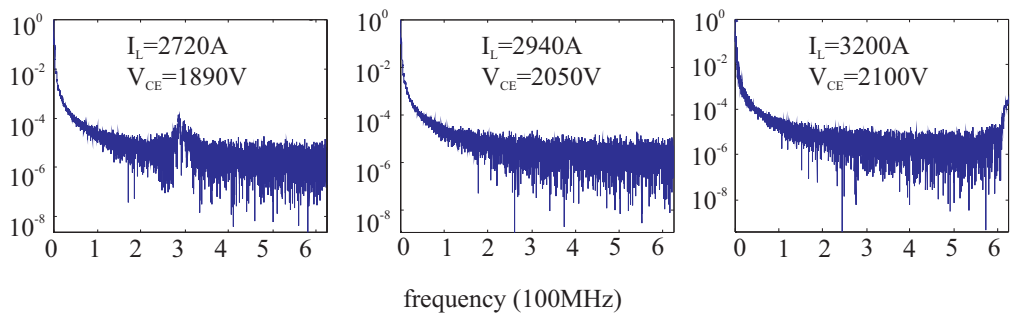

Figure 4.5: Discrete Fourier transform (FFT) of antenna signal of MS3: variation of DC-link voltage and load current 


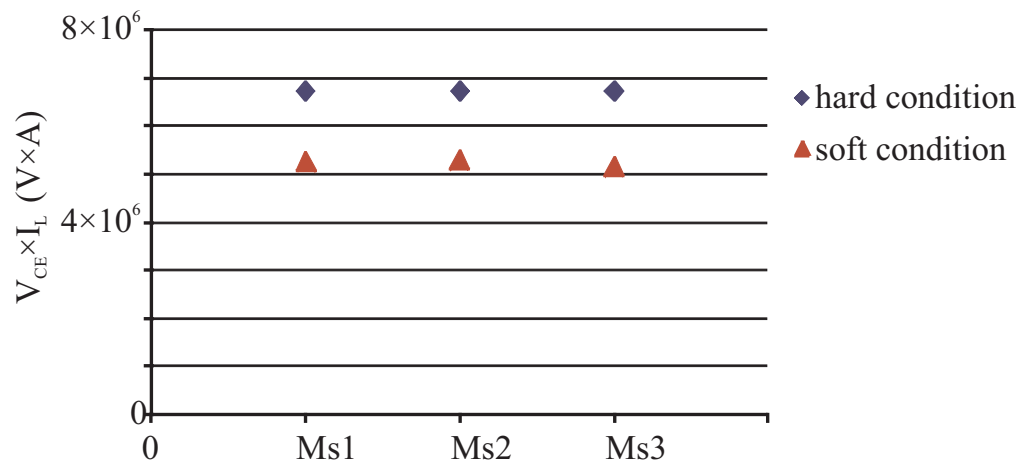

Figure 4.6: Product of $V_{D C}$ and $I_{L}$ as the TA-oscillations occur

$\left(I_{L} \times V_{D C}=5.3 \times 10^{6} \mathrm{VA}\right)$, lower current and (or) lower voltage, is identified to be about $300 \mathrm{MHz}$. As shown in Fig.4.6 the product of $I_{L}$ and $V_{D C}$ is found to be a decisive parameter for the oscillations in all the three measurement series.

The analytical estimation in chap.2 helps to understand the effect of $V_{D C} \times I_{L}$. As Eq.(2.13) represents, the maximal e-field $E_{\max }$ of the SCR is proportional to $\sqrt{I_{L} \times V_{D C}}$. This indicates the relevance between the avalanche generation and the TA-oscillation. To reach the critical e-field, the $W_{S C R}$ is roughly inversely proportional to the current of the IGBT. Therefore the lower current of IGBT means wider SCR and longer transit time of the SCR and accordingly the lower frequency of the transit time effect.

Further measurements show that under certain conditions more partial frequencies are even found in a single turn-off process of the IGBT module. Fig.4.7 shows for example a finely resolved diagram of the TA-oscillation. Beside the frequencies of $300 \mathrm{MHz}$ and $625 \mathrm{MHz}$, a partial frequency of $430 \mathrm{Mhz}$ is even found to be the dominant frequency of the oscillation. The analysis in the Chap. 3 shows that, for the IMPATT mechanism, RFpower generation on at least two frequencies is possible. Together with 

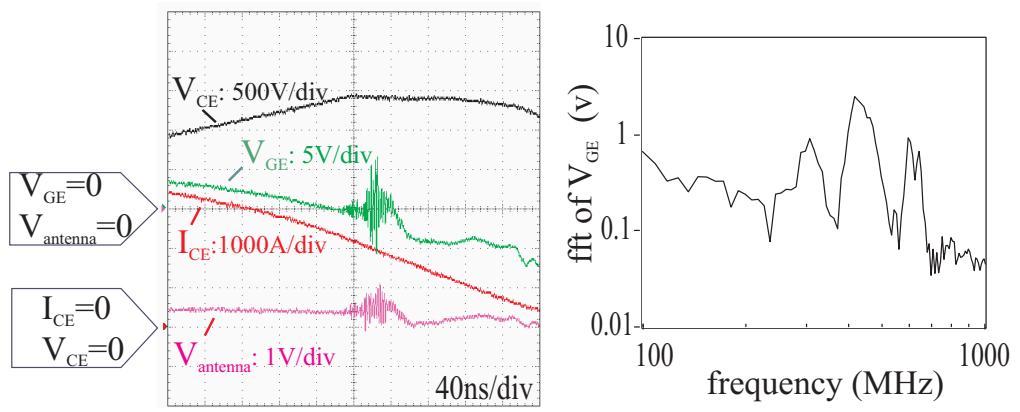

Figure 4.7: Multi frequency TA-oscillations during the Module turn-off

the possible hole transit time effect, the frequencies population of TAoscillation can be more complicated.

\section{Effect of temperature}

The dynamic Avalanche oscillation was reported in the similar frequency range of several hundreds of $\mathrm{MHz}$ as TA-oscillation [LSG98]. As discussed in chap.2.4.2, such oscillations are quite sensitive to the temperature. The following measurements are processed to verify, if TA-oscillation is affected by the temperature similarly as the dynamic IMPATT oscillations. In this connection, the junction temperature is varied from $25^{\circ} \mathrm{C}$ to $125^{\circ} \mathrm{C}$. MS3 as shown in Fig.4.2 is processed at each temperature. The oscillation of $625 \mathrm{MHz}$ becomes weaker due to the raise in temperature. In order to reproduce the $625 \mathrm{MHz}$ oscillation at $80^{\circ} \mathrm{C}$, the product of $I_{L}$ and $V_{D C}$ must be adjusted $30 \%$ higher than the test at $40^{\circ} \mathrm{C}$. The condition to reproduce the $300 \mathrm{MHz}$ oscillation increased by about $10 \%$ when the temperature rises from $40^{\circ} \mathrm{C}$ to $80^{\circ} \mathrm{C}$. For the temperature above $80^{\circ} \mathrm{C}$, no $625 \mathrm{Mhz}$ oscillation can be detected. Due to the limitation of over voltage by the IGBT blocking capability, we have to dispense with the further increase of the current. The $300 \mathrm{MHz}$ oscillations are still found at $125^{\circ} \mathrm{C}$. Fig. 4.8 shows the conditions of the oscillation accordingly to the temperature variation. The 


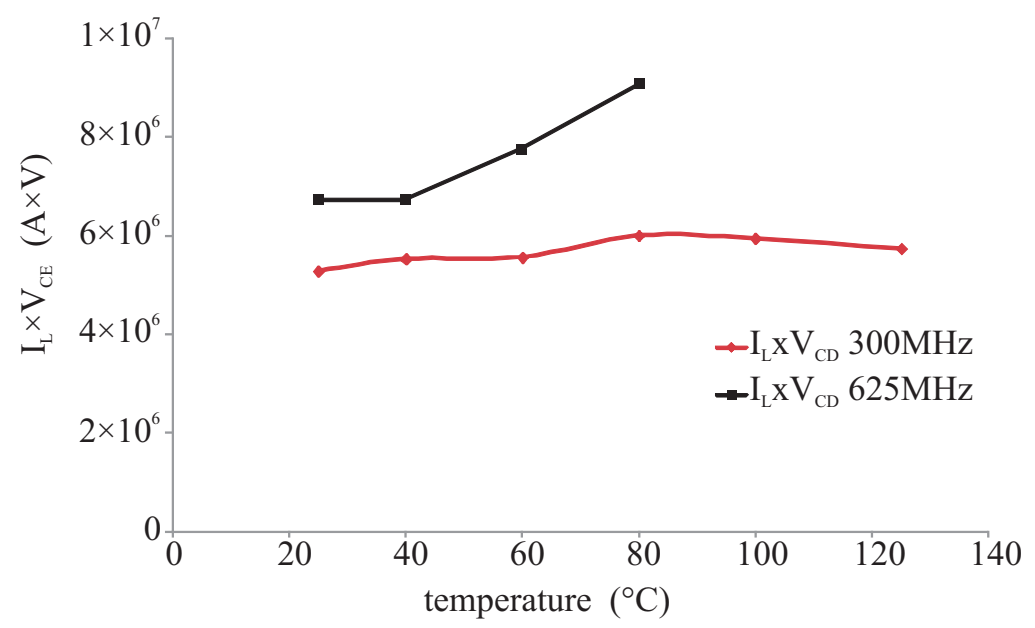

Figure 4.8: Start conditions of the oscillation corresponding to the temperature

dynamic avalanche oscillation is caused by the K-centre and can be suppressed by the temperature variation of a few degrees. No such strong temperature sensitivity can be demonstrated for TA-oscillation. The causes of the two oscillation types are not the same.

The discussion in Chap. 3 shows that the coefficient of the impact ionization decreases about $1 / 4$ when the temperature rises from $40^{\circ} \mathrm{C}$ to $80^{\circ} \mathrm{C}$, that can be read out Fig.3.2. This agrees with the temperature sensitivity of the $625 \mathrm{MHz}$ oscillations. The $300 \mathrm{MHz}$ oscillations do not show such temperature sensitivity.

\subsubsection{Measurement of TA-oscillation on a substrate and single chip}

The early investigations show that the PETT oscillations are found on the parallel switching of IGBTs and diodes [MGS02] [GMS02]. To investigate the effects of paralleling chips on TA-oscillations, additionally to the 
engineering IGBT module with $24 \times$ paralleling IGBTs, measurements on substrate setups with $4 \times$ IGBTs, $2 \times$ IGBTs and also on setups with single IGBT chip are conducted. The TA-oscillations are even found on the single IGBT setups. The paralleling of chips is not absolutely necessary for TA-oscillation.

The following measurements on the substrate setups are carried out to find out the critical parameter, the amplifier, the resonant circuits of TA-oscillation.

\section{Effect of $R_{G}$ on substrate}

To eliminate the effect of the asymmetry in the gate circuit of the IGBT Modules, measurements are processed on the substrate setups with much simpler gate circuit than the whole engineering module. Fig.4.9 shows a substrate setup with $4 \times$ IGBT- and $4 \times$ diode-chips arranged on a DCB. Each IGBT chip is connected to an anti-parallel diode via emitter wire bonds. This substrate setup works as a single switch.

During the varying of gate resistor from $6.8 \Omega$ to $15 \Omega$, all the other conditions are kept unchanged. Fig.4.10 shows the traces of the gateemitter voltage. The amplitude of TA-oscillation increases with $R_{G}$ till $R_{G}=10 \Omega$ and then reduces with the further increase of $R_{G}$. No oscillation is found in the range of $R_{G} \leq 6.8 \Omega$ and $R_{G} \geq 15 \Omega$.

Smaller $R_{G}$ leads to the faster turn-off of the MOS channel. The resulted higher net hole density at the emitter side leads to a steep raise of $V_{C E}$. Therefore the varying of $R_{G}$ has a similar effect as the varying of the $I_{L}$ in the reverse direction.

\section{Effect of cross wire bonding between IGBT chips on a substrate}

According to Zimmermann patent, shown in Fig.2.10, and [Gut06], the Cross wire bonds between the adjacent IGBTs were proved to be an effective manner to suppress the PETT oscillations by mismatching the reso- 


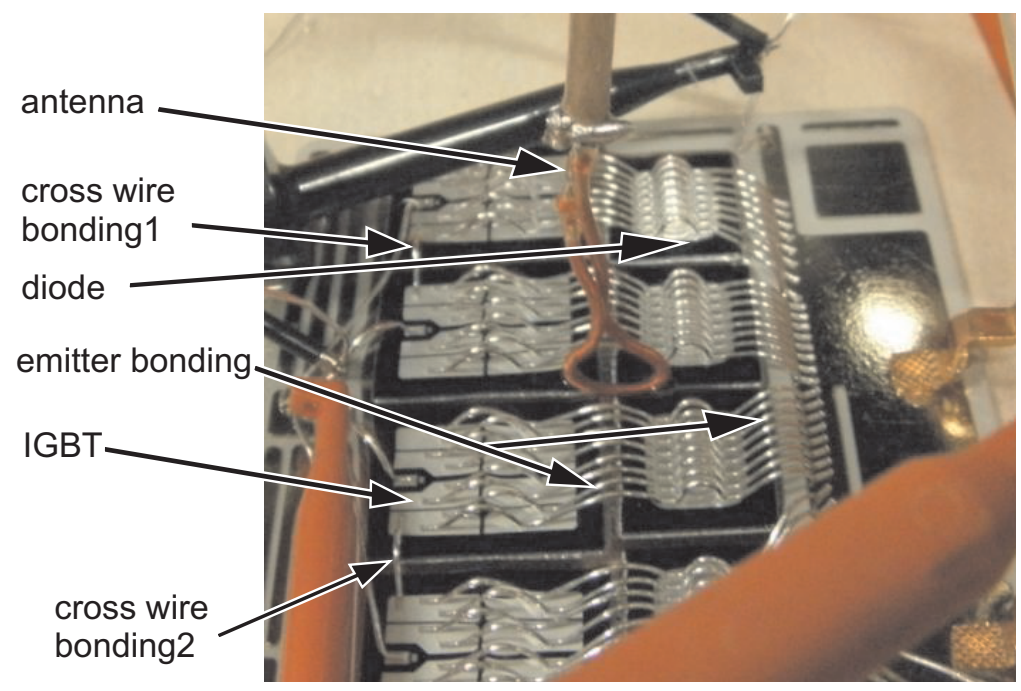

Figure 4.9: Substrate setup with $4 \times$ IGBT- and $4 \times$ diode-Chips

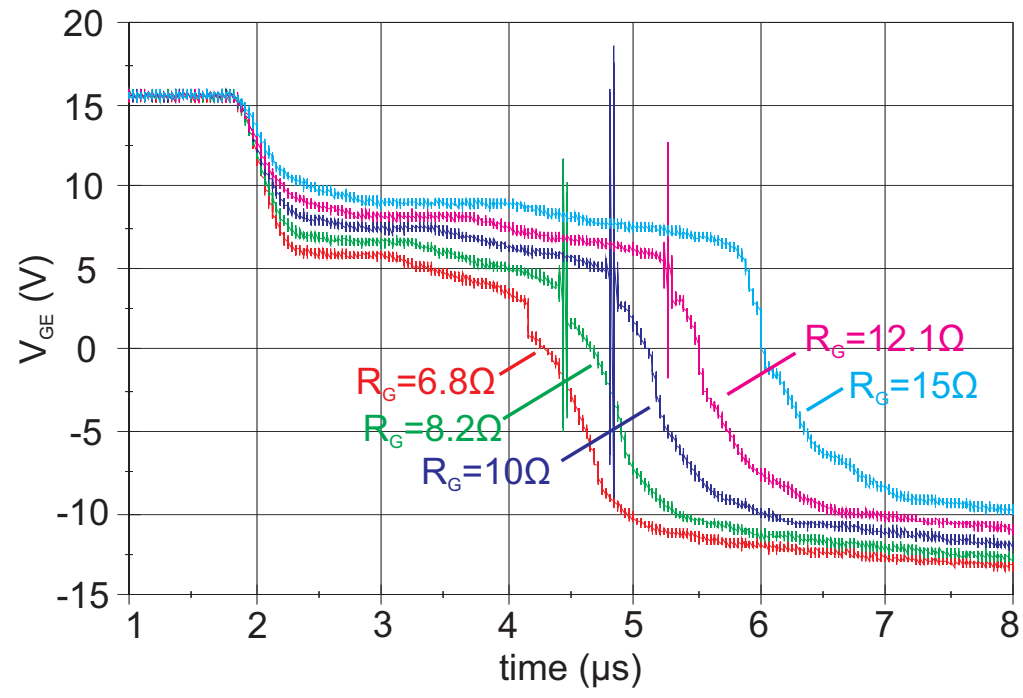

Figure 4.10: RF-oscillations dependence on $R_{G}$ 
nant circuit frequency and the PETT frequency. As the TA-oscillations are found in the similar frequency range as PETT oscillations, the cross wire bonds were also expected to suppress TA-oscillations. Fig.4.11 shows the measurements on substrates with different cross wire bond configurations.

A $30 \mathrm{MHz}$ oscillation is found on the substrate which can be suppressed by the cross wire bond 1 and 2 between the adjacent IGBTs as shown in Fig.4.9. The $600 \mathrm{MHz}$ oscillation is however not obviously suppressed by the cross wire bonds. As discussed in Chap.??, during the time the PETT normally happens, the SCR in IGBT and diode vary only within a narrow range. The eigenfrequencies of the resonant circuits can be shifted out of the transit time frequency of PETT mechanism by the cross wire bond. But the TA-oscillation occurs during the raise of $V_{C E}$ and fall of the $I_{C E}$. The SCR is expanding much more dynamically than during PETT oscillation. During TA-oscillation, because of the expanding of SCR, both the transit time frequency and the resonant frequencies are changing within a wide range. This makes it difficult to mismatch the resonant circuit to the transit time frequency by cross wire bonds.

\section{Effect of chip thickness}

In case of the PETT oscillation, the oscillation occurs at the end of the tail current phase. The SCR has reached almost the field stop layer on the back side of the IGBT. Therefore the transit time frequency should be inversely proportional to the width of the base region of IGBT. To vary the base region IGBT chip types with identical front side and back side structures but different thickness are processed. Substrates with $4 \times$ IGBTs, as shown in Fig.4.9, were setup for each IGBT types. Fig.4.12 shows the discrete Fourier transform (DFT) of the TA-oscillation signals measured on the substrate with different IGBT chips. Oscillations of $475 \mathrm{MHz}$ are found at all the four chip types. No influence of the chip thickness can be identified. This indicates that, as the TA-oscillations occur, the SCRs do not reach 


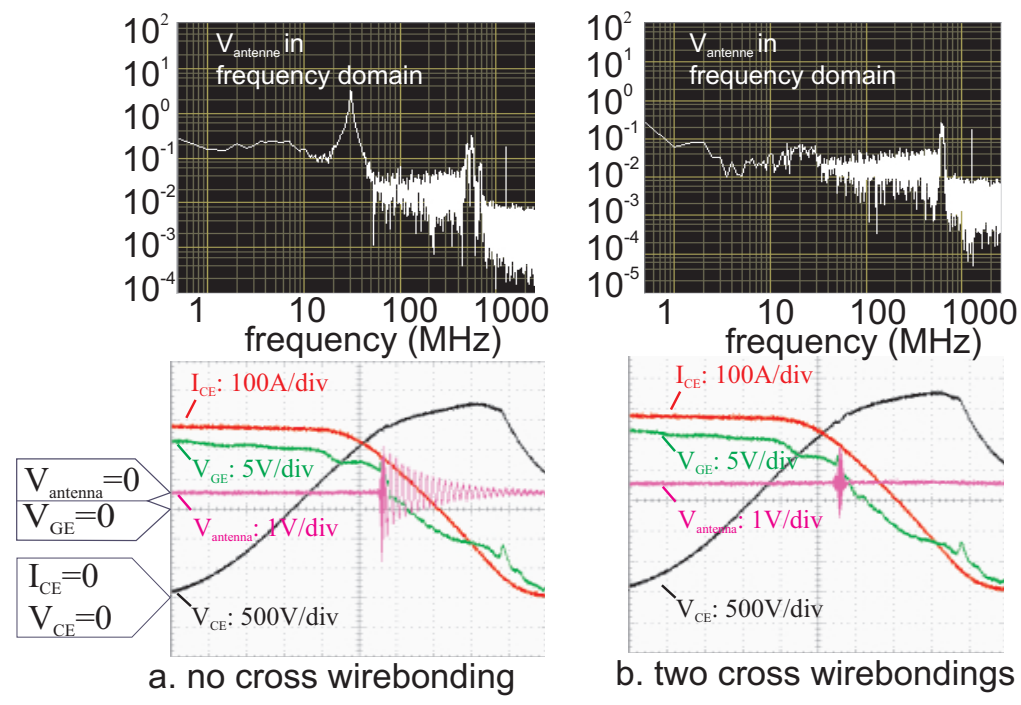

Figure 4.11: a. TA-oscillations on substrate without cross wire bond. b. TA-oscillations on substrate with two cross wire bonds

the field stop of all chips.

\section{Source of the oscillation signal}

As the TA-oscillation was first observed on the gate-emitter voltage of the IGBT module, the active participation of gate was assumed. To find out if the the gate emitter voltage actively participates in the TA-oscillation, it is necessary to measure the chip internal gate emitter voltage during TA-oscillation. As a gate resistor is already integrated on the IGBT chip, a special sense contact as shown in Fig.4.13 was constructed to pick up the potential between the gate- and the emitter-metallization. At the end of a BNC cable two feather needles are contacted. The distance between the needles is adjusted so that one of them contacts on the gate and the other on the emitter-metallization. The loop area between the two needles is kept as small as possible to avoid the EMI coupling. For the single chip 

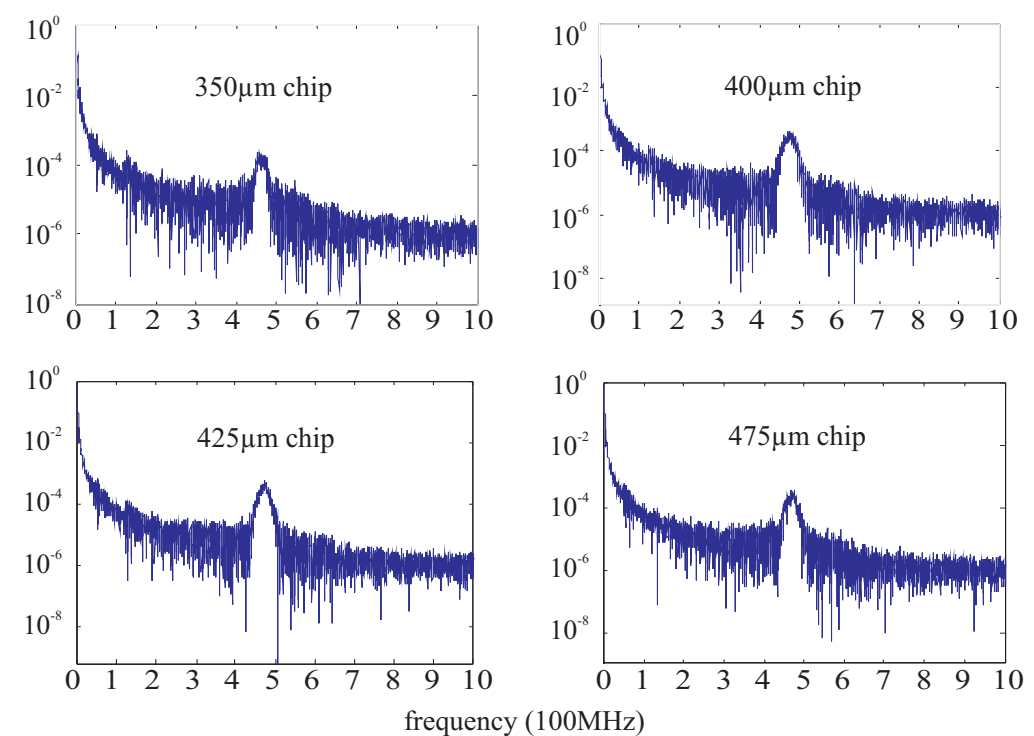

Figure 4.12: TA-oscillations are measured at $I_{L}=500 \mathrm{~A}, V_{C D}=2500 \mathrm{~V}$ under $25^{\circ} \mathrm{C}$ 


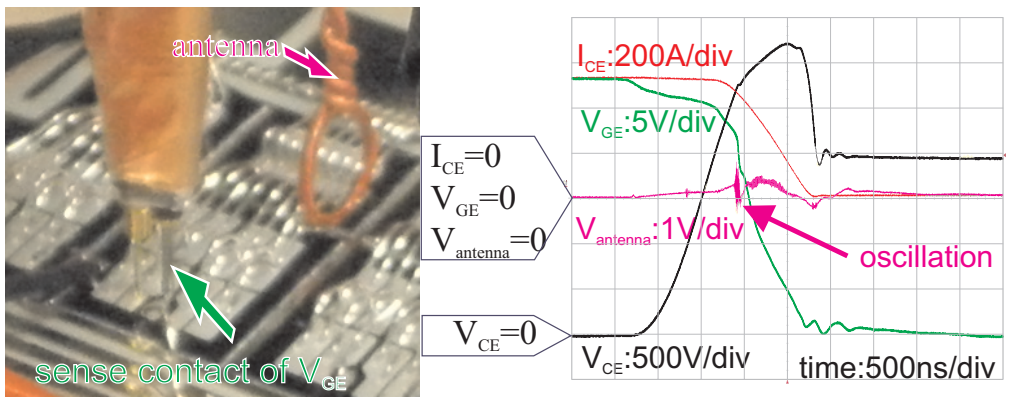

Figure 4.13: Sense contact to measure the The internal gate-emitter voltage $V_{G E I N}$ is directly on the IGBT chip with needle sense contact, oscillation on the antenna and no oscillation on the gate is observed.

measurements, just one IGBT and one diode on the substrate setup are connected. The signal of antenna and the internal gate voltage $V_{G E I N}$ of the IGBT are shown in Fig.4.13. As compared to the oscillation on the antenna, no high frequency disturbances can be found on the $V_{G E I N}$. As TA-oscillation happens, the internal gate-emitter potential is driven to under the threshold voltage of the IGBT. Therefore the previously detected oscillation signal on $V_{G E}$ is just passive raised by the EMI coupling. The active participation of $V_{G E}$ in TA-oscillation can not be demonstrated.

Furthermore varying the relative position and direction of the antenna to the emitter wire bonds cause the strong influence on amplitude of the measured oscillation signals. The oscillation signal received by the antenna parallel to the wire bonds (Fig.4.14) is more than three times stronger than that received by the perpendicular antenna (Fig.4.15). This indicates that emitter wire bonds are part of the resonant circuit of TA-oscillation. The wire bonds act as antenna and emitted the RF-oscillation signals. 


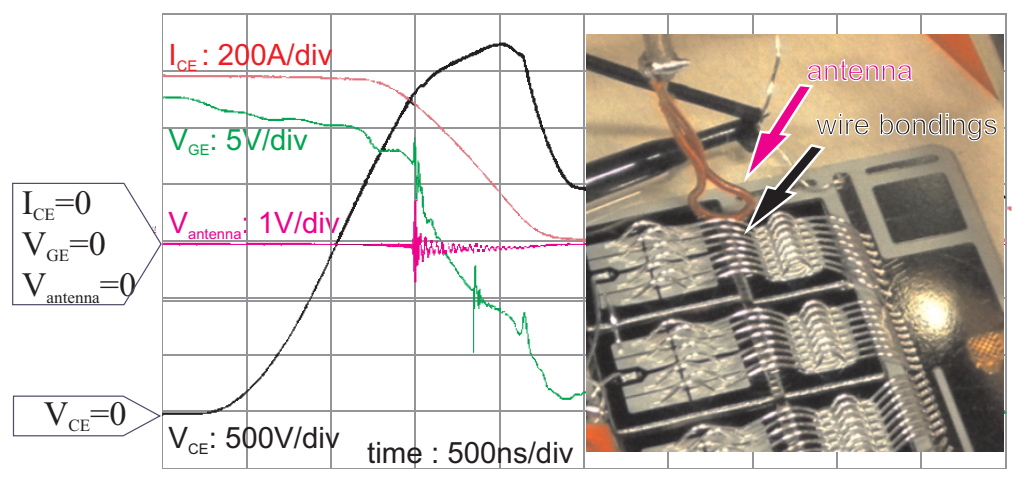

Figure 4.14: Measurement of the TA-oscillation with the antenna parallel to the emitter wire bonds

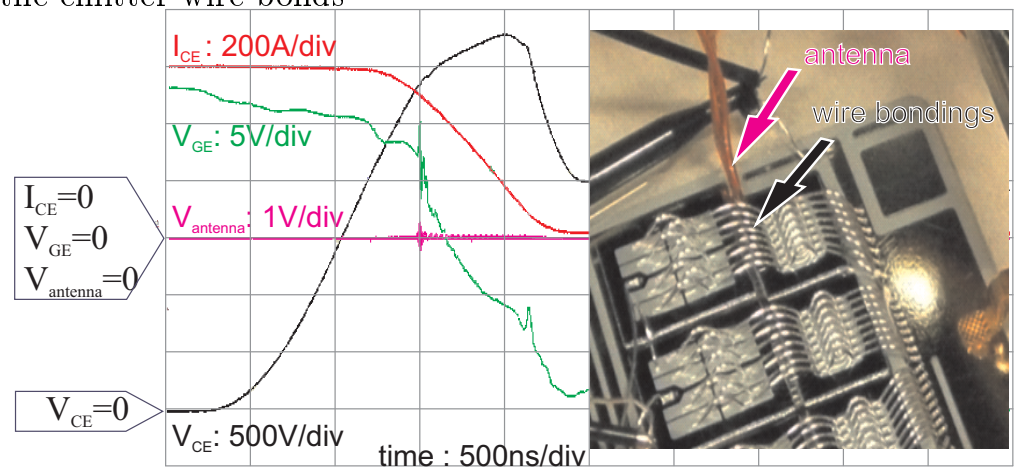

Figure 4.15: Measurement of the TA-oscillation with the antenna perpendicular to the emitter wire bonds 


\section{Effect of the wire bond configuration}

After the emitter wire bonds are found to be part of the resonant circuit of TA-oscillation, measurements are processed on different wire bond configurations, as shown in Fig.4.16. Fig.4.16 shows the test setups with different configurations of wire bonds. On DUT1 in Fig.4.16(a), the IGBT and the anti parallel diode are directly connected by the emitter wire bonds. On DUT2 in Fig.4.16(b), the IGBT and the anti parallel diode are connected via a metal island of the DCB top layer. Both DUTs are turned off at a current density of $130 \mathrm{Acm}^{2}$, which is out of the safe operating area. Fig. 4.18 shows the measurement on DUT1. Oscillations are found at the begin of the dynamic avalanche process. Fig.4.17 shows the result of the measurement on DUT2. Oscillations are found at the end of the dynamic avalanche process. The frequencies are determined as $600 \mathrm{MHz}$ on DUT1 and $155 \mathrm{MHz}$ on DUT2. The strong influence of the emitter wire bonds is demonstrated.

\section{Typical characters of TA-oscillation}

After all the measurements on the $3.3 \mathrm{kV}$ IGBT setups, the typical characters of TA-oscillations can be summarized as the following:

- TA-oscillations are found during the IGBT turn-off under high load current and high DC-link voltage and during the dynamic avalanche.

- The gate resistance has to be in a certain range.

- Large stray inductance in the current commutation circuit leads to high over voltage and thus has the similar effect on TA-oscillation as the high DC-link voltage.

- Longer emitter wire bonds leads to lower frequency of TA-oscillations

- TA-oscillations are found at the junction temperature from $25^{\circ} \mathrm{C}$ to $125^{\circ} \mathrm{C}$. 

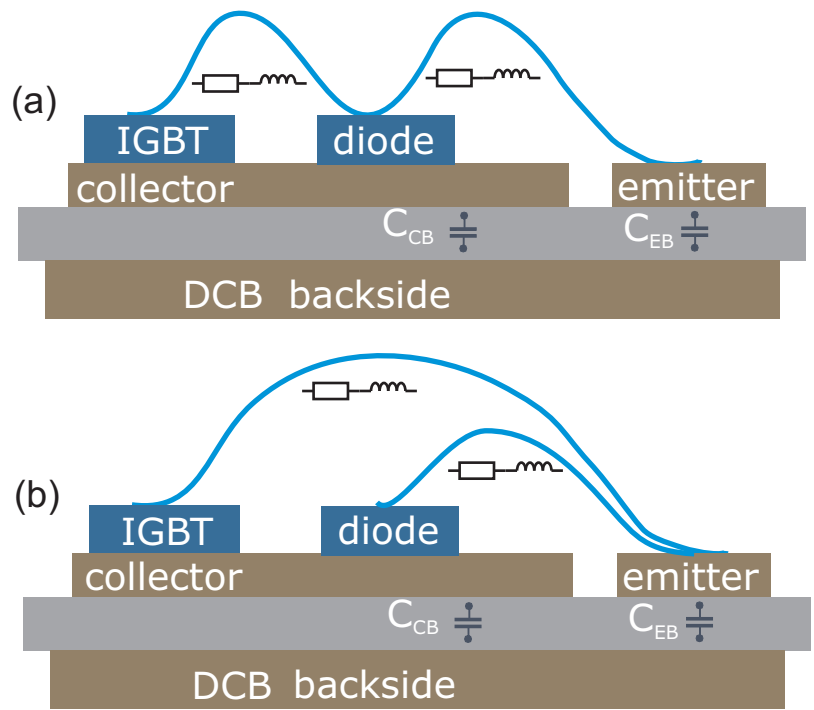

Figure 4.16: (a) Direct wire bonding between IGBT and diode (DUT1) (b) Indirect wire bonding between IGBT and diode (DUT2) 


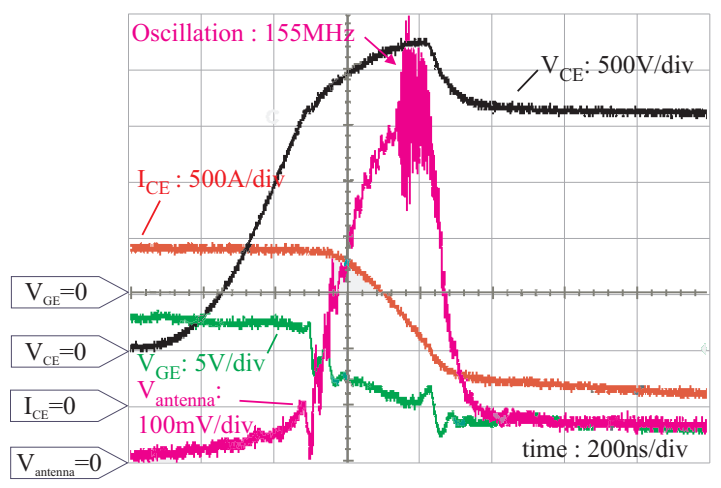

Figure 4.17: Measurement on DUT2 with long emitter wire bonds. HFoscillation at end of the dynamic avalanche, oscillation frequency is about $150 \mathrm{MHz}$

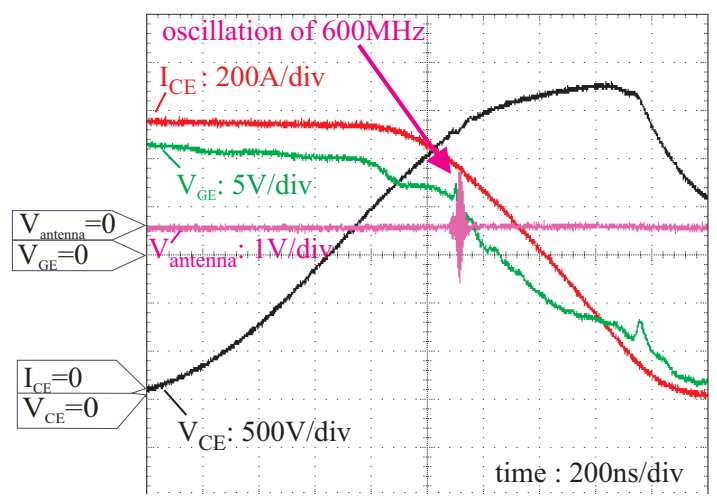

Figure 4.18: Measurement on DUT1 with direct emitter wire bonds. HFoscillation at the begin of dynamic avalanche, oscillation frequency is about $600 \mathrm{MHz}$ 
- TA-oscillations are found on all chip types with the thickness from $350 \mu \mathrm{m}$ to $475 \mu \mathrm{m}$, and the frequencies of TA-oscillations are found to be the same.

- Paralleling of IGBTs is not necessary for the TA-oscillations. TAoscillations are found also on setups with a single IGBT and a single diode.

- The frequencies of TA-oscillations are found in a frequency band form 100 to $800 \mathrm{MHz}$ for $3.3 \mathrm{kv}$ IGBTs. Multi frequencies are found in a single turn-off process.

- The cross wire bond between the adjacent IGBTs is effective for the suppression of the low frequency oscillation but not effective for the high frequency oscillation.

- The oscillation signals on $V_{G E}$ are proved to be EMI coupling. As TA-oscillation happens, the internal gate emitter voltage has already dropped to below the threshold voltage. The gate did not participate actively in TA-oscillation.

- The emission source of the oscillation signals are proven to be the emitter wire bonds.

\subsection{Analysis of the resonant circuits}

Resonant circuit is necessary to raise the TA-oscillation. Due to the high frequency of the oscillation, the resonant circuit must consist of small inductors and capacitors. And they must be located close to the chips, and the capacitance of the chip itself is expected to participate in the oscillation. Fig.4.19 shows a schematic illustration of the resonance circuits near to the IGBT chip with the participation of emitter wire bondings. Three 


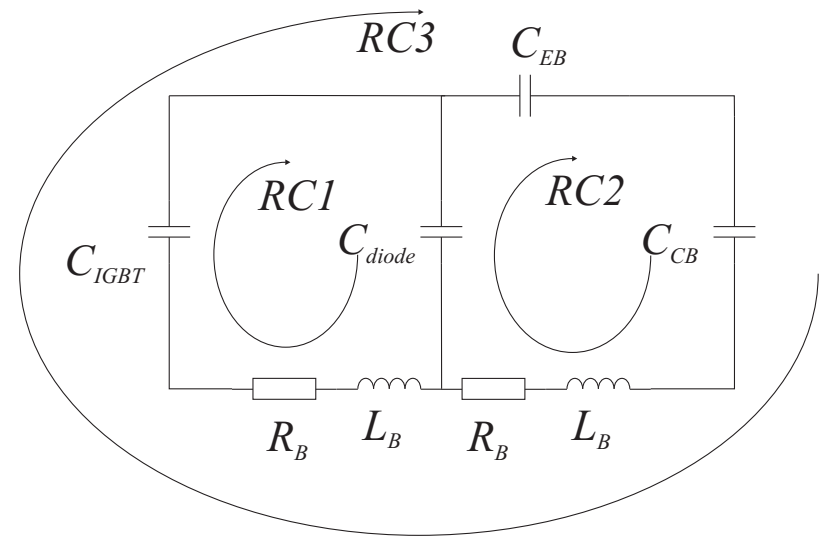

Figure 4.19: Resonance circuits $(R C 1, R C 2$ and $R C 3)$ of the DCB setups shown in Fig.4.16(a)

resonance circuits are constituted of IGBT and diode capacitance, capacitance between DCB areas and the inductance of bond-wires. Resistances in the circuit act as damping factor and have just minimal impact on the resonance frequencies. For the quantitative analysis of complex structures, such as substrate and even the total IGBT modules, simulation tools auch as Q3D and HFSS are developed by ANSYS to assistant the design analysis using FEM.

\subsubsection{Inductance of wire bonds}

Due to its reliability and flexibility, wire bondings are the most popular method to realize the internal electrical connections of power modules. The parasitic inductance, electric resistance as well as the thermal resistance are critical parameters that limit the design and application of power modules. The inductance of wire bonding is one of the key parameter for the analysis of the resonant circuit of the TA-oscillations. For the resonant circuit analysis of setup with just one IGBT and one diode, the parasitics 
simplification steps to estimate the wire bonding inductance

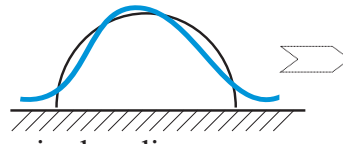

wire bonding

half circle
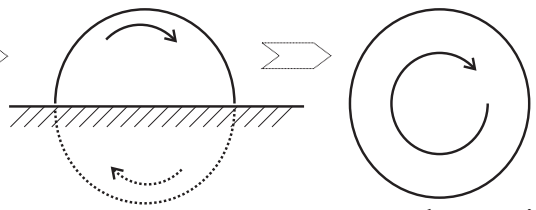

a conductor ring

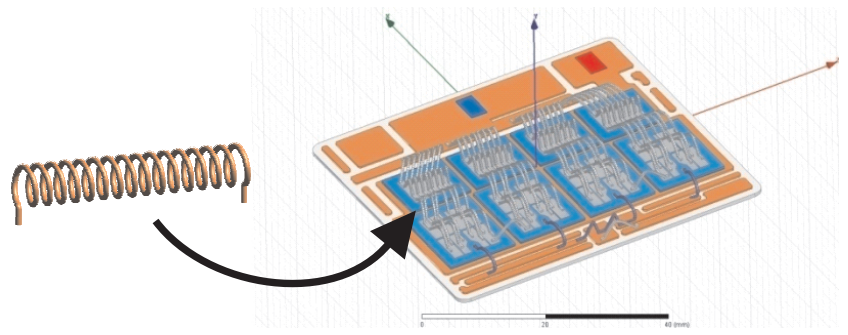

Figure 4.20: Analytical estimating of the wire bonding inductance

can be analytically estimated. Although the analytical estimation is not so precise as the FEM simulations, but it is a practice way to find a start point of the frequency analyse by means of network simulations. The wire bondings in the power modules are always arranged close above a conductor surface such as the DCB-copper layer. One of the emitter wire bonding configuration is shown for example in Fig.4.9. Theoretically for high frequency analysis, the copper layer can be taken als ideal conductor. The electric and the magnet-field caused by a wire bonding over a copper layer is equivalent to the field caused by two wire bondings arranged antisymmetric to the copper surface.(Mirror principle theoretical electro technique) Fig.4.20 shows the method to estimate the inductance of the emitter wire bondings analogous to the air coil. 


$$
\begin{aligned}
W_{w b} & =\frac{1}{2} W_{c} \\
W_{c} & =\frac{1}{2} L_{s c} I_{c}^{2} \\
W_{w b} & =\frac{1}{2} L_{w b} I_{w b}^{2} \\
I_{c} & =\frac{1}{N} I_{w b}
\end{aligned}
$$

A emitter wire bonding can be firstly approximated by a half circle wire conductor, whose electrical magnet field energy is equal to half of a wire ring. The electro-magnet field energy of the parallel arrangement of the wire bonding $W_{w b}$ is equivalent to the half electro-magnet field energy of the coil $W_{c}$, Eq.(4.1) (4.2) and (4.3), with the current ration $1 / N$ as expressed in Eq.(4.4). Here $\mathrm{N}$ is the winding number of a coil and the number of the parallel wire bondings. $I_{c}$ is the coil current, this means the current in a single bond wire. And $I_{w b}$ is the total current of the parallel wire bondings.

For long coil with $l>>r$, as in case of the emitter wire bonding on the substrate as in Fig.4.9, the inductance $L_{l c}$ is calculated as in Eq.(4.5) [MRP05]. For the short coils with $l>0.6 r$, as in case of the wire bonding of a single chip, the inductance $L_{s c}$ is given by Eq.(4.6) [MRP05].

$$
\begin{aligned}
& L_{l c}=N^{2} \frac{\mu_{0} \mu_{r} \pi r^{2}}{l} \\
& L_{s c}=N^{2} \frac{\mu_{0} \mu_{r} \pi r^{2}}{\sqrt{l^{2}+4 r^{2}}}
\end{aligned}
$$

The inductance of the emitter wire bonding can be therefore estimated 
Table 4.1: Parasitics extracted for the resonant circuit analysis

\begin{tabular}{|c|c|c|}
\hline parasitics & value & unit \\
\hline$L_{B}$ & 2.65 & $\mathrm{nH}$ \\
\hline$C_{E B}$ & 140 & $\mathrm{pF}$ \\
\hline$C_{C B}$ & 10 & $\mathrm{pF}$ \\
\hline
\end{tabular}

analogues to a coil by Eq.(4.7), which is eliminated out of the equations of Eq.(4.1) and (4.6).

$$
L_{w b}=\frac{1}{2} \frac{\mu_{0} \mu_{r} \pi r^{2}}{\sqrt{l^{2}+4 r^{2}}}
$$

The inductance of the emitter wire bondings on the example substrate setups as shown in Fig. 4.16, is estimated as approximately $2.7 \mathrm{nH}$, which agrees well with the result of the Q3D simulation. Although in the real applications the magnet coupling to the adjacent layout structures must be considered, this analogous inductance estimation is still an effective method to find out a start point for the network analysis of the resonant circuit in IGBT module.

The capacitances of the DCB copper areas can be calculated as conventional plate capacitor according to Eq.(4.8). For the further analysis, the parasitics of the resonant circuits as shown in Fig.4.19 are estimated and listed in Tab.4.1.

$$
C_{D C B}=\frac{\epsilon_{0} \epsilon_{r} A}{D}
$$

\subsubsection{Differential capacitance of IGBTs and diodes}

As mentioned in Chap.2, the differential capacitance corresponding to the actual SCR can be defined as Eq.(2.9) for the resonant circuit analysis. 
The chip capacitances in the resonant circuits as shown in Fig.4.19 are estimated according to the actual width $W_{S C R}$ of the space charge regions in the respective chips.

Although IGBTs are so called voltage controlled semiconductor switches, But their switching processes are indeed current controlled. For the IGBT, the expanding of the $S C R$ and the ramp up of $V_{C E}$ is mainly limited by the net hole density, which is determined by the load current and the ratio between the electron current and hole current in SCR. For the different turn-off conditions, such as different $R_{G}$ and different $I_{L}$ the expansion of $S C R$ in the IGBT and the diode can totally differ from each other. By the assumption that the $W_{S C R}$ in the diode is $\gamma$ times of the $W_{S C R}$ in the IGBT, the chip capacitances an the the corresponding resonance frequencies can be calculated as shown in Fig.4.21 for the analytical understanding. Due to the absence of plasma in the diode, $\gamma$ is generally larger than one. Thereby it is taken into account that chip capacitance reaches its minimum after the SCR reaches the field stop layer, and the active area of the diode is roughly $50 \%$ of the IGBT. Higher $I_{L}$ and smaller $R_{G}$ lead to faster switching process. The faster is the switching process of the IGBT the larger is $\gamma$.

Fig.4.22 shows the diagram of the eigen frequencies of the three resonant circuits, estimated according to the $W_{S C R}$ in IGBT at $\gamma=2$, together with the transit time frequencies of the corresponding SCR. The transit time frequencies decrease with the expanding of SCR. The resonance frequencies increase due to the decrease of the chip capacitances given by (2.9). The intersections of group A and group B in the figure represent the frequencies, at which the oscillations can be effectively amplified by the transit time mechanisms. In the frequency range above $1 \mathrm{GHz}$ oscillations is theoretically possible, but such oscillations are not jet observed the real measurements. During the switching off of the channel current, the ration between the eletron current and hole current in the IGBT and 


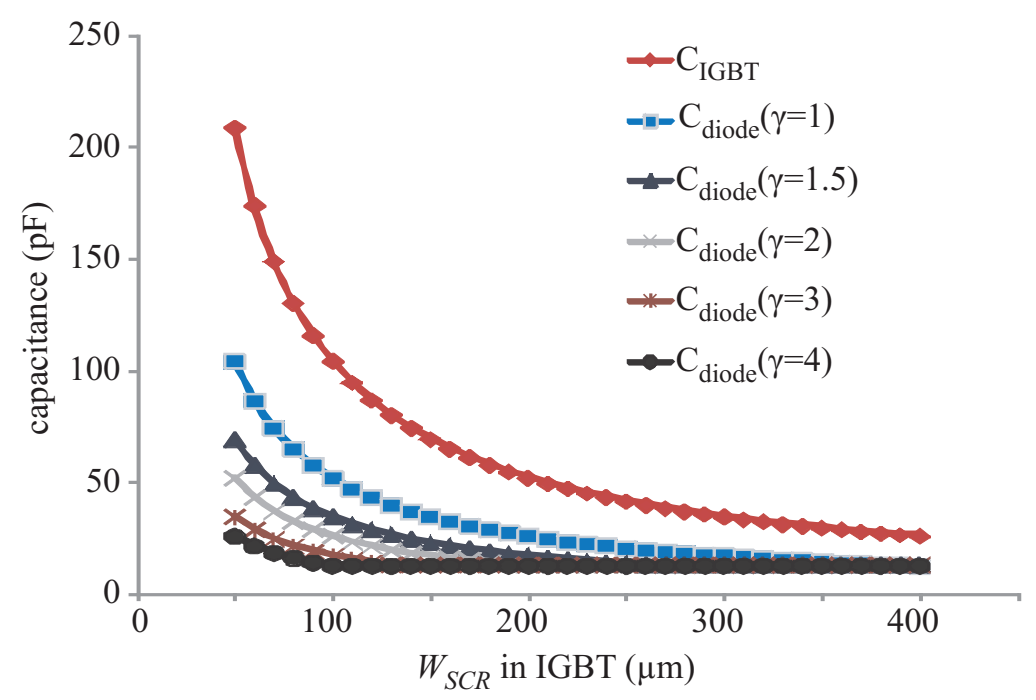

Figure 4.21: Chip capacitance of IGBT and diode according to $W_{S C R}$

the corresponding $\gamma$ is not constant. So that the analytical estimation of the resonant frequencies just helps to the understanding of the oscillation mechanisms. The device simulations are indispensable for the fine quantitative analysis of the dynamic IGBT turn off process.

\subsection{Device simulations}

Compared to measurements, simulations gives more flexible way to analyse the oscillation mechanisms and allow the access to the internal process of the IGBT chips. Similarly to the measurement a $3.3 \mathrm{kV}$ field stop IGBT cell with trench gate structure on emitter side is created for the device simulation [Pfi12]. To accelerate the simulation process, just half of cross section of a IGBT cell is constructed as 2D model for the cylinder symmetrical simulation. Doping profiles are adjusted so that the blocking capability, forward bias and short circuit characteristic fit to the measurements on 


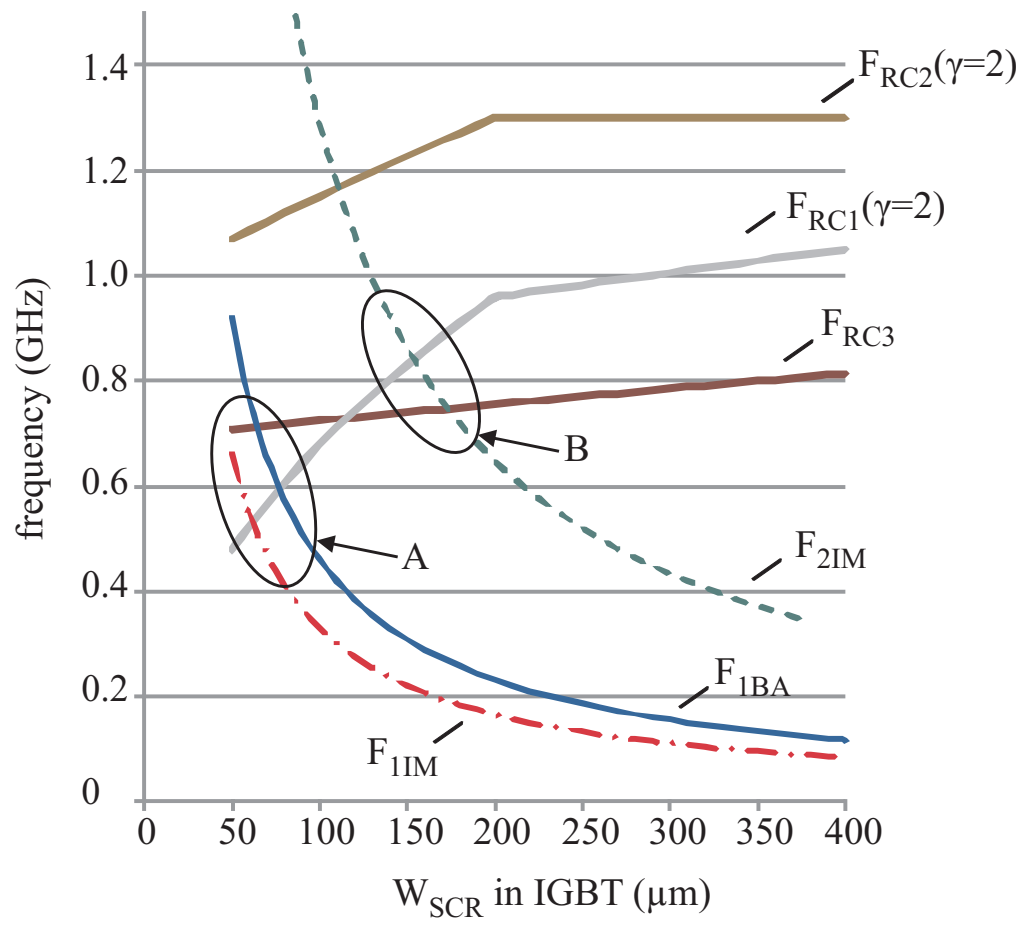

Figure 4.22: Intersection between eigenfrequencies of the transit time mechanism and the resonant frequencies during the expanding of $W_{S C R}$ 


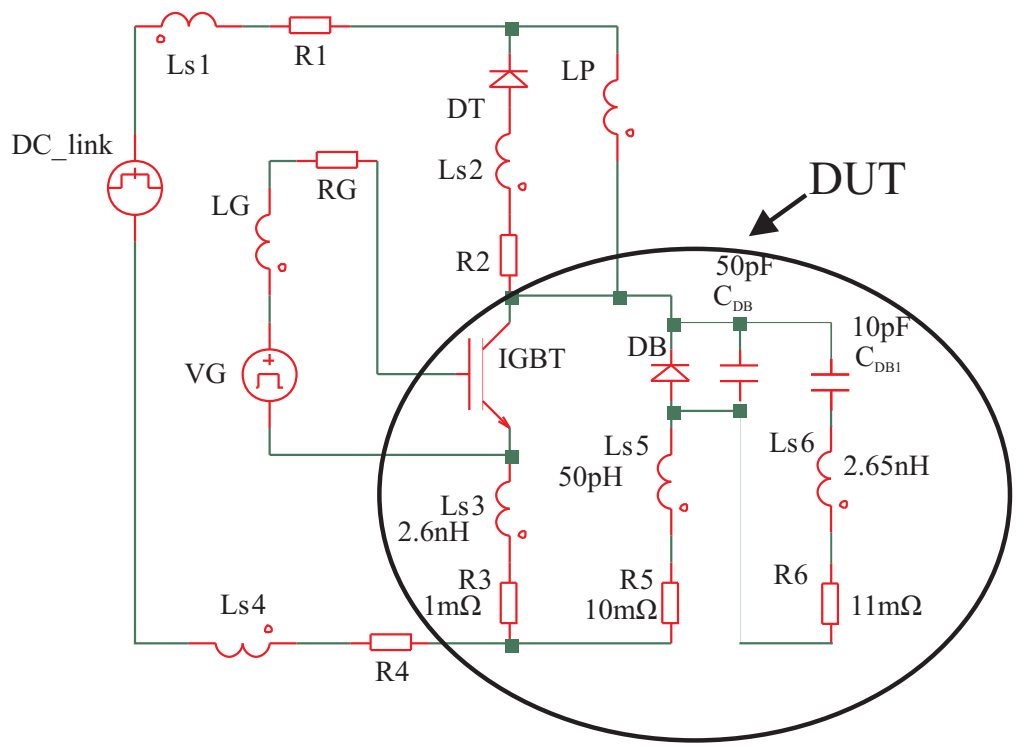

Figure 4.23: Circuit of Device-Simulation, a 2D model of half IGBT cell is integrated in the circuit

the IGBT modules.

\subsubsection{Circuit}

The rapid development of the computer techniques makes it now possible to solve the semiconductor device model in a spice-similar circuit environment [syn11]. For the oscillation analysis the resonant circuit is just as important as the proper device model of the IGBT chip, therefore the realistic estimation of parasitic components in the module and substrate setup is necessary to find the start point for the TA-oscillation analysis. To reproduce the resonant circuits near the IGBT chips, the parasitics on the DCB setups are extracted analytically and verified by Q3D simulations.

The total circuit of the system simulation is shown in Fig.4.23. The device IGBT model builds first together with the parasitics of the substrate 
setups a single IGBT switch (DUT), which consists of three resonant circuits as shown in Fig.4.19. Ls3 and $R 3$ together with $L s 5$ and $R 5$ represent the wire bonding between the IGBT and the diode, and the $L s 6$ and $R 6$ represent the wire bonding between the diode and the copper area on the top of the DCB. The series switching of $C_{C B}$ and $C_{E B}$ is simplified as $C_{D B 1}$. And $C_{D B}$ represents the junction capacitance of the diode $D B$. The DUT is again integrated in the test circuit corresponding to labour setup for the measurement. The entire test process is solved by Sentaurus device simulator. In contrast to the previous HF-oscillation simulations, the turn-off process is simulated without artificial HF-stimulations. The maximal time step during the IGBT turning-off is limited to $0.2 \mathrm{~ns}$, which is found to be a good compromise between the simulation time and the convergence. The time derivative of the current signal on R5 represents the antenna signal in the measurement.

Oscillations are observed on the current of R5 during the whole ramp up process of $V_{C E}$. The frequencies of the weak oscillation decrease according to the extension of the SCR from $560 \mathrm{MHz}$ to $200 \mathrm{MHz}$ before the strong oscillation occurs. At the time of MOS-channel turned-off, the strong oscillation occurs with the frequency of around $600 \mathrm{Mhz}$. Oscillations with similar frequency trend can also be observed on the fine resolved $V_{C E}$ and $I_{C E}$.

\subsubsection{Effect of resonant circuit's eigenfrequencies}

The varying of $C_{D B 1}$ shows a significant effect on the oscillation signals. The frequencies of the oscillations reduce according to the increase of $C_{D B 1}$. The maximal amplitude is found at $610 \mathrm{MHz}$ and $C_{D B 1}=22 \mathrm{pF}$ as shown in Fig.4.25(a). The circuit configurations of the device simulation are analysed by network simulation parallel to the device simulation. As the network simulator does not solve the semiconductor equations, the parameters such capacitance and inductance of the semiconduc- 


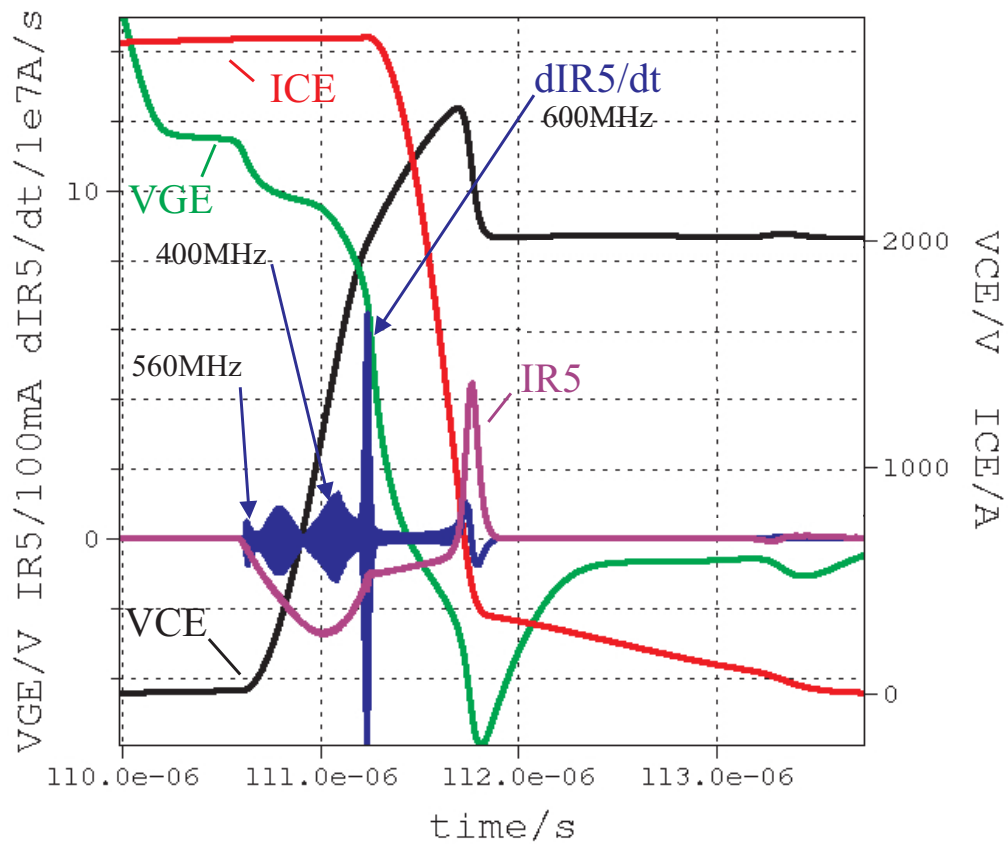

Figure 4.24: Oscillations are found during the turn-off simulation. 


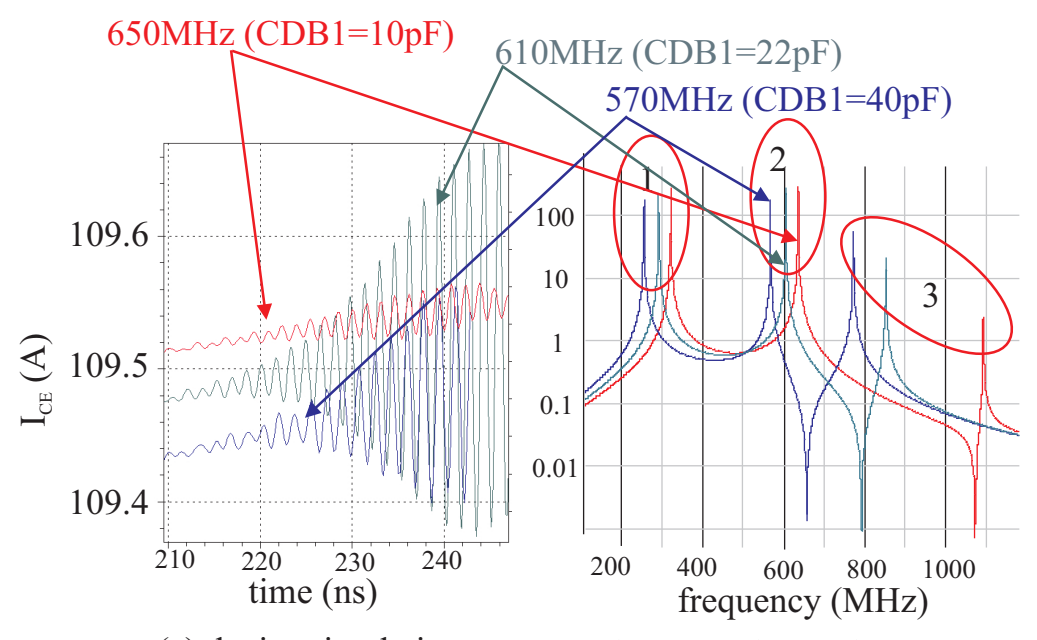

(a) device simulation

(b) network simulation

Figure 4.25: Compare the device simulations with the network simulations at $C_{D B 1}=10 \mathrm{pF}, 22 \mathrm{pF}$ and $40 \mathrm{pF}$

tor chips must extracted manually according to the actual SCR. For the time point of $600 \mathrm{MHz}$ oscillation, at $111.2 \mu \mathrm{s}$ in Fig.4.24, the combination of $C_{I G B T}=100 \mathrm{pF}$ and $L_{I G B T}=1 \mathrm{nH}$ is found as proper configuration by comparing the Simetrix simulation and the device simulations. Three eigenfrequency groups are calculate using SIMetrix as shown in Fig.4.25(b). Group 2. lies around $600 \mathrm{MHz}$, which matches well the oscillation frequencies of the device simulations.

Further simulations with diverse configurations of $C_{D B 1}$ and $C_{D B}$ are processed both in device simulations and in circuit simulations. The diagram in Fig.4.26 shows the device simulations and SIMetrix simulations with various of resonant circuit configurations. The amplitude and the frequencies of device simulation show the same tendency as the network simulations. The simulation time and the expense of the network simulation using SIMetrix is significantly lower compared to device simulation. The network simulation is therefore attractive for the module designer to 


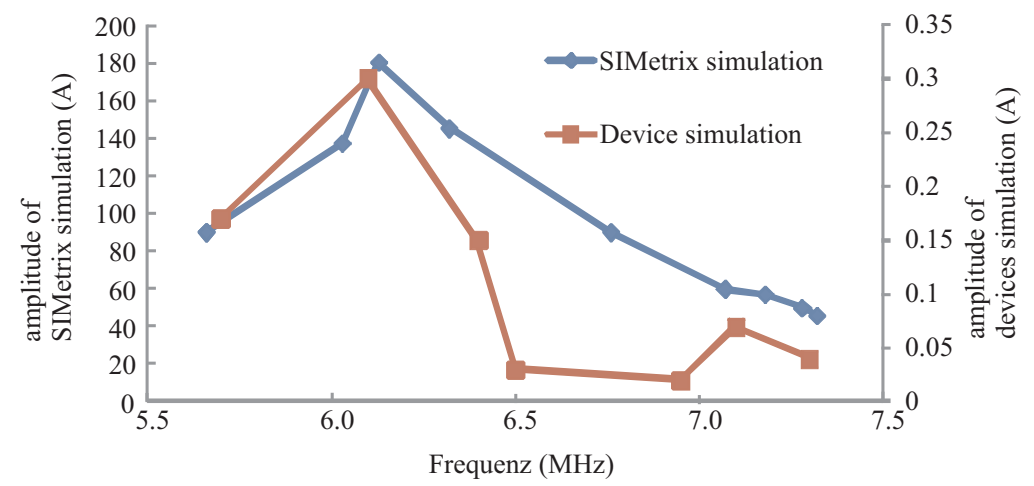

Figure 4.26: Device simulations show the same trend as the network simulations with the same $C_{D B 1}$ and $C_{D B}, C_{I G B T}=100 \mathrm{pF}$ and $L_{I G B T}=1 \mathrm{nH}$ are used for IGBT circuit model

optimize the module layout in respect to the oscillations as the chip capacitance and the chip inductance are dependent on the $S C R$ in the chip. The combination of $C_{I G B T}=100 \mathrm{pF}$ and $L_{I G B T}=1 \mathrm{nH}$ is not valid for universally. For the module layout optimization, the parameters of the equivalent chip model need to be extracted for the different work conditions and different time points.

\subsubsection{Effect of load current}

The effect of load current has been theoretically analysed in Chap.2. The higher load current means the higher carrier densities in the IGBT and smaller $R_{G}$ leads to faster turn off of the MOS channel. The resulted higher hole current and therefore higher hole density in the space charge region drive the the maximal e-field $E_{\max }$ to the critical value $E_{c}$ at a smaller $W_{S C R}$. This leads to a small transit time and a higher transit time frequency. The possible oscillations may be raised when the transit time frequencies cross the eigenfrequencies of the resonant circuits, for example near the intersections as shown in Fig.4.22. To verify this theory, 
the load current $I_{L}$ is varied from $18 \mathrm{~A} / \mathrm{cm}^{2}$ to $181 \mathrm{~A} / \mathrm{cm}^{2}$.

The oscillation peak is first found at $56 \mathrm{Acm}^{2}$ with the frequency of $400 \mathrm{Mhz}$, then reduces with the increasing $I_{L}$ and then reaches its second peak amplitude at $109 \mathrm{Acm}^{2}$ with the frequency of $650 \mathrm{MHz}$. Fig. 4.28 shows the expansion of SCR in the IGBT device model at the moment of the oscillations. The oscillation frequencies of the device simulation match well with the measurements. For $I_{L}=56 \mathrm{~A}, W_{S C R}=155 \mu \mathrm{m}$ as the $400 \mathrm{Mhz}$ oscillation happens. For $I_{L}=109 A, W_{S C R}=205 \mu \mathrm{m}$ as the $650 \mathrm{MHz}$ oscillation happens. The eigenfrequencies of the resonant circuit can be read out in Fig.4.22 with indications of work points group A for $400 \mathrm{MHz}$ and work points group B for $650 \mathrm{MHz}$ oscillations according to the current $W_{S C R}$. During the trun-off, the expanding of SCR leads to the decrease of transit time frequencies of IGBT and increase of the resonance frequencies on the substrate. The transit time frequencies match the resonance frequencies first time at around $400 \mathrm{MHz}$ near the work points group $\mathrm{A}$ in Fig.4.22. As the dynamic avalanche does not occure, the $400 \mathrm{MHz}$ oscillation is induced mainly by PETT mechanisms. The further expanding of the SCR leads temporarily to the mismatch between the transit time frequencies and the resonance frequencies and decay of the oscillation. For $I_{L}=109 A$, as the SCR expands to about $200 \mu m$, the dynamic avalanche happens. The transit time frequencies match the resonance frequencies at $650 \mathrm{MHz}$ near the work points group B in Fig.4.22. The frequencies of the simulated oscillations are lower than the analytical estimated frequencies. The possible reason for the deviations between the oscillation frequencies and the estimated resonant frequencies is the time-delay of the avalanche region in IGBT, which is not involved in the simplified resonant circuit model in Fig.4.19. The equivalent circuit of the avalanche region for microwave diode has been derived analytically by Gilden and Hines [GH66]. Although the avalanche region can be described by the parallel switching of a inductance $L_{a}$ and a capacitance $C_{a}$, but they are strong 


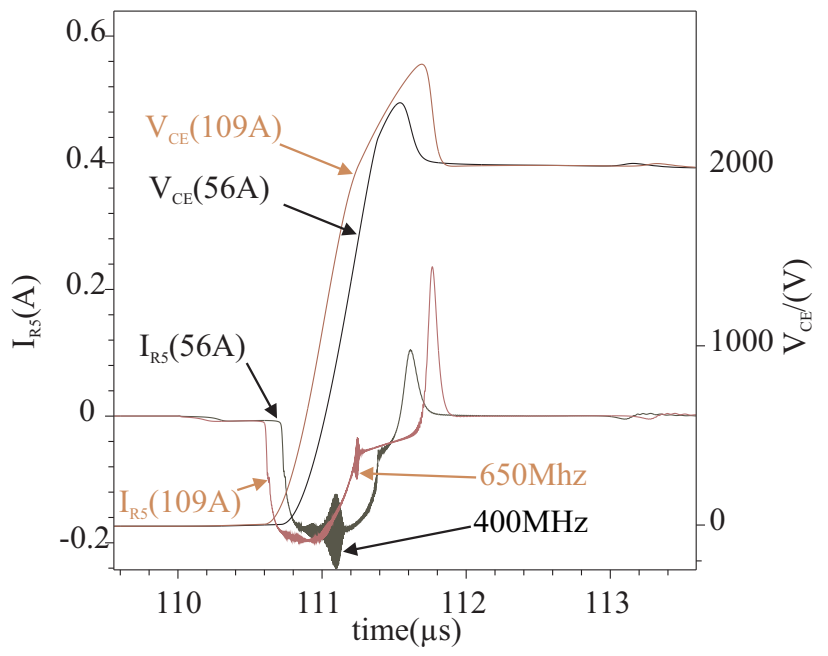

Figure 4.27: Simulation of the dependency on load current

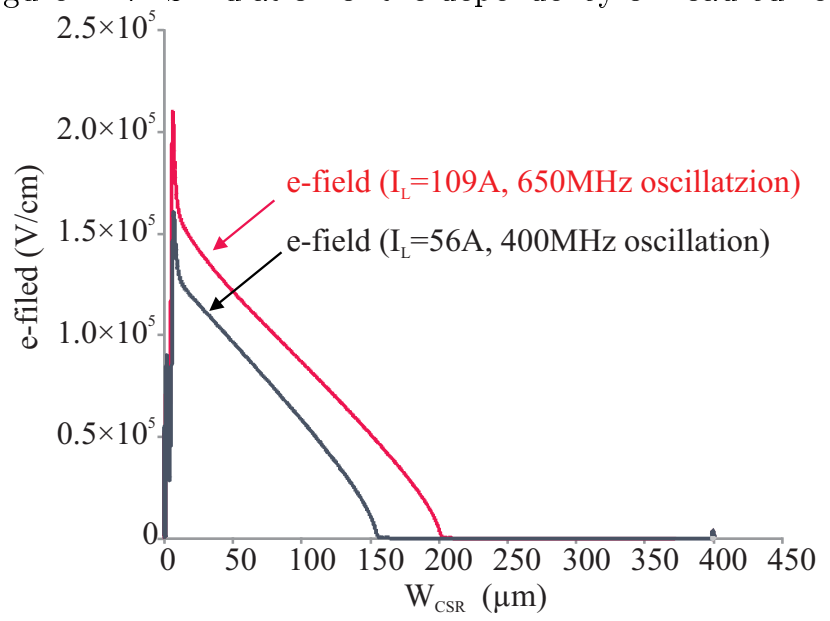

Figure 4.28: $W_{S C R}$ in the IGBT at the moment of the oscillations of 400Mhz and $650 \mathrm{Mhz}$ 
Table 4.2: Simulated oscillations by $I_{L}=109 A$ with varying of $R_{G}$

\begin{tabular}{|c|c|c|}
\hline$R_{G}(\Omega)$ & $F_{O}(\mathrm{MHz})$ & $I R 5_{A C}(\mathrm{~A})$ \\
\hline 5 & 600 & 9 \\
\hline 11 & 590 & 0.5 \\
\hline 17 & 705 & 80 \\
\hline 30 & 680 & 3 \\
\hline
\end{tabular}

non-linear dependent on the $E_{\max }$. Due to the dynamic changing of $E_{\max }$, it is difficult to find the proper equivalent circuit of the avalanche region analytically for each time during the turn off of IGBTs.

\subsubsection{Effect of gate resistance}

The gate resistor limits the current in the gate circuit so that the switching off of the MOS channel can be delayed by larger gate resistors. Using a smaller gate resistor the electron current would be turned off faster and would lead to earlier and higher hole current on the emitter side during the IGBT turning-off. Simulation results of varying $R_{G}$ from $5 \Omega$ to $40 \Omega$ in Tab.4.2 show that the amplitudes of oscillation are sensitive to $R_{G}$ during the frequencies are moderately shifted by the different $R_{G}$ configurations. This phenomenon is also found by the measurements. Under certain conditions, when the transit time frequencies match well with the resonance frequencies, the simulations can not run till the end due the divergence. The maximal amplitude is found as $80 \mathrm{~A}$ at $705 \mathrm{Mhz}$ and $R_{G}=17 \Omega$, which would be strong enough to destroy the device. In this case, the E-field in IGBT shows a strong deformation compared to a triangle and indicates a TRAPATT (trapped plasma avalanche-triggert transit) similar process. 

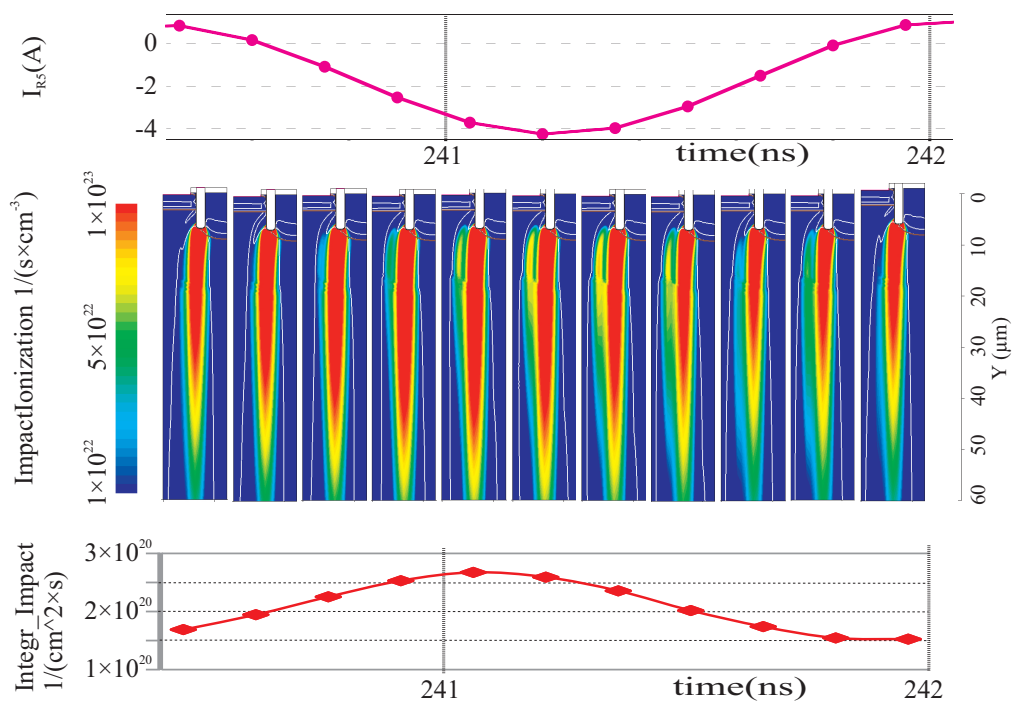

Figure 4.29: Impact ionization and charge injection during TA-oscillation

\subsubsection{Internal process of IGBT chips}

Device simulations make it possible to get a view on the internal state of the IGBT chip. Fig.4.29 shows the impact ionization process within one period of the TA-oscillation. During the TA-oscillation, 10 time points of the current in R5 are shown on the top of the figure. The corresponding internal states of IGBT chip are shown in the middle of Fig.4.29, in which the periodical change of the impact ionization can be observed. The integration of the impact ionization over the SCR during TA-oscillation is shown on the bottom of Fig.4.29, which indicates a periodical current injection during the avalanche process.

The e-field distributions in the SCR, corresponding to the time points during the TA-oscillation, are shown in Fig.4.30. The sinusoidal overlapping can be observed on the triangle e-field distributions. The differences of the e-field between the individual time points and the average value of 
one oscillation period are defined as Eq.(4.9). In Fig.4.31 a propagating wave of $\Delta E(x, n)$ is clearly visible. As shown in Fig.4.32 and Fig.4.33, the propagating waves are also observed on the electron distribution and hole distribution in the SCR. The wave is induced by the drift of electrons packet from emitter side to collector side with the velocities of $8.56 \mathrm{~cm} / \mathrm{s}$. And the wave of holes spreads in the reverse direction with the velocity of $7.75 \mathrm{~cm} / \mathrm{s}$. The velocities comply with the effective saturation velocities of electrons packet and holes packet in the SCR respectively. The variation of the eDensity in the SCR is much stronger than that of the hDensity, which indicates the dominant roll of the electron transit time effect in the TA-oscillation.

$$
\begin{gathered}
\Delta E(x, n)=E(x, n)-\overline{E(x, n)} ; n=1,2, \ldots, 11 \\
\Delta e \operatorname{Density}(x, n)=e \operatorname{Density}(x, n)-\overline{e \operatorname{Density}(x, n)} ; n=1,2, \ldots, 11 \\
\Delta h \operatorname{Density}(x, n)=h \operatorname{Density}(x, n)-\overline{h \operatorname{Density}(x, n)} ; n=1,2, \ldots, 11
\end{gathered}
$$

\subsubsection{Effect of impact ionization}

The flexibility of device Simulation allows to answer the question, if the TA-oscillation is solely caused by the avalanche. After that the oscillation of $600 \mathrm{Mhz}$ is reproduced by the reference simulation, the simulation is repeated with deactivated impact ionization. Although it is not possible to turn off the impact ionization in real IGBT chips, this comparison is the simple method to verify, if the TA-oscillation is solely caused by the impact ionization. Fig.4.34 shows two simulations with identical parameter settings except of the impact Ionization. On the top left is the turn- 


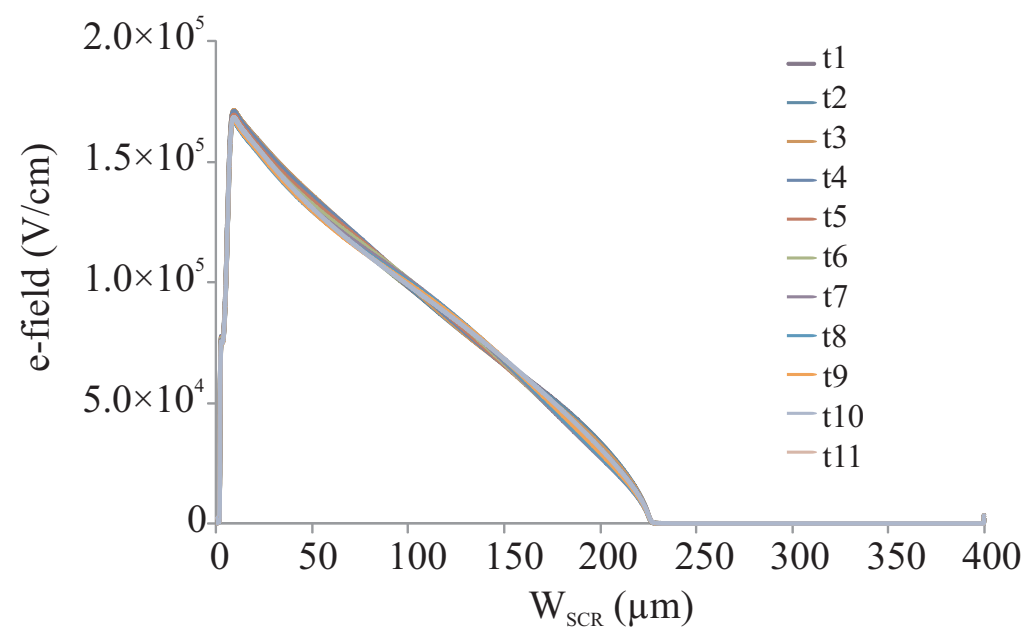

Figure 4.30: e-field distribution in IGBT during TA-oscillation

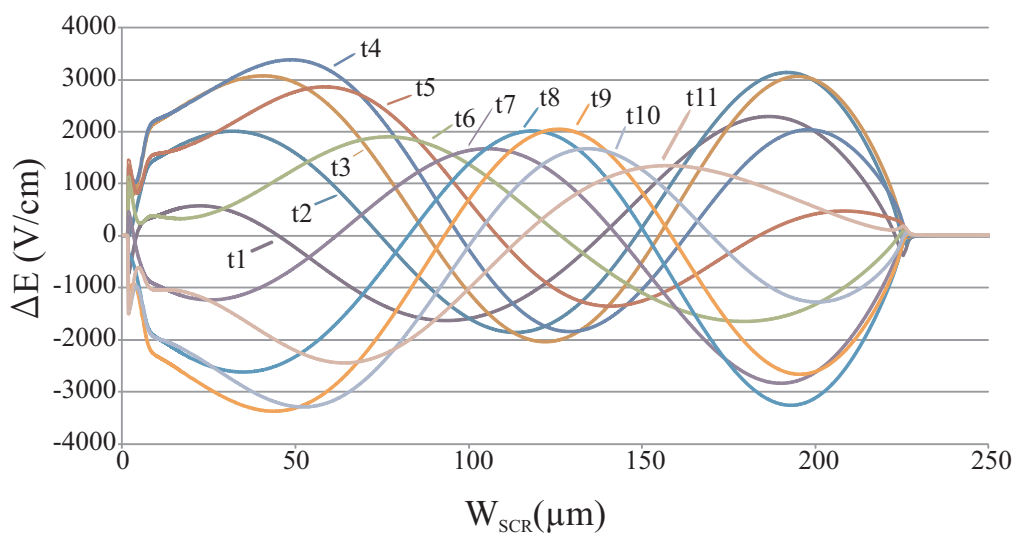

Figure 4.31: E-filed variation during TA-oscillation according to Eq.(4.9) 


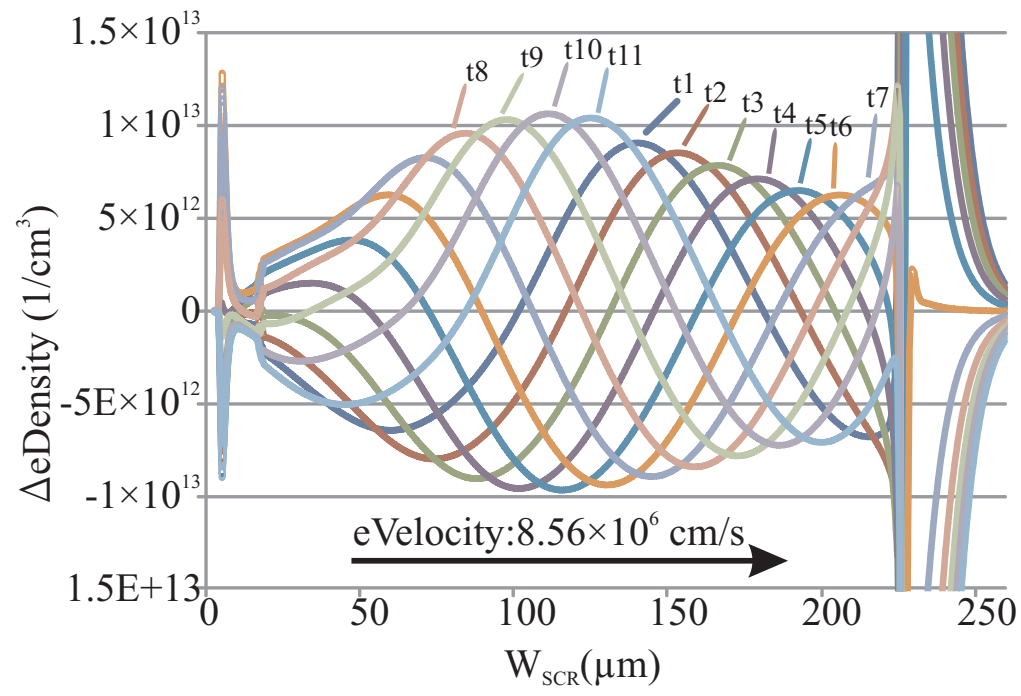

Figure 4.32: eDensity variation in the SCR of IGBT during TA-oscillation according to Eq.(4.10)

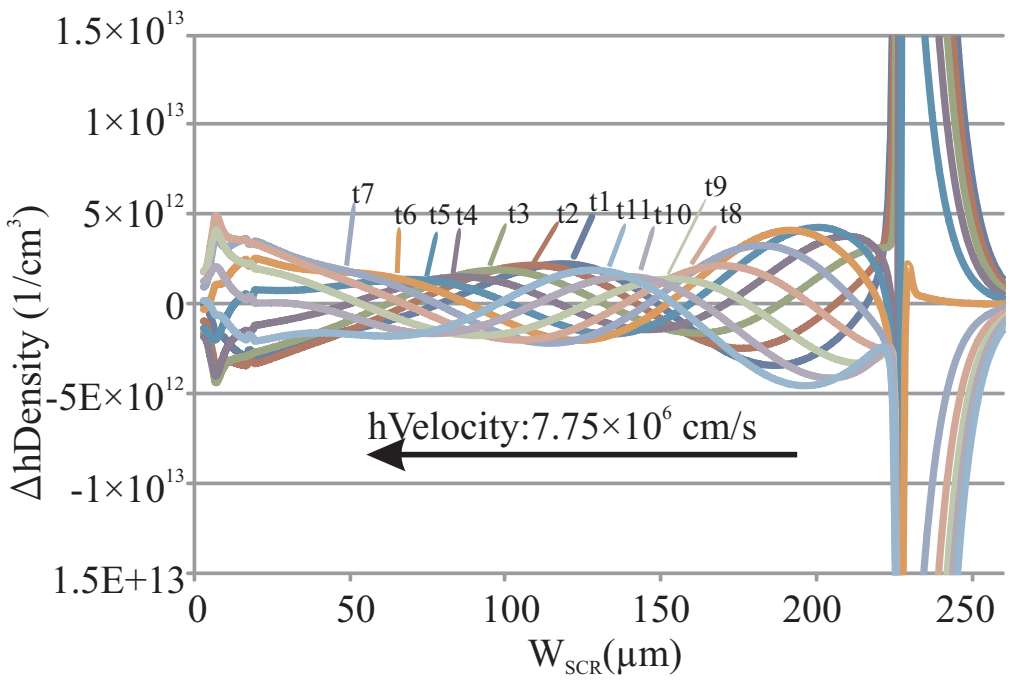

Figure 4.33: hdensity variation in the SCR of IGBT during TA-oscillation according to Eq.(4.11) 


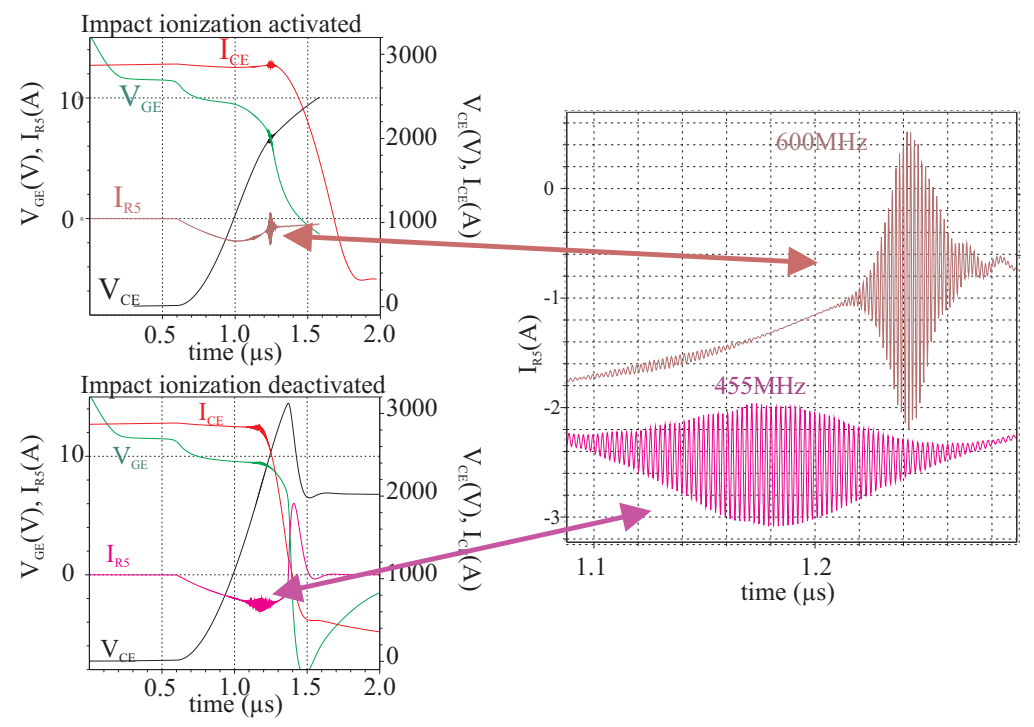

Figure 4.34: Simulation of the effect of impact ionization on TA-oscillations

off diagram of the reference simulation, in which the impact ionization is allowed. On the left bottom side is the diagram of the simulation, in which the impact ionization is deactivated. The simulation with deactivated impact ionization shows a $455 \mathrm{MHz}$ oscillation with a half of the amplitude of the $600 \mathrm{Mhz}$ oscillation. The $455 \mathrm{Mhz}$ oscillation occurs $60 \mathrm{~ns}$ earlier and its duration is three time longer than that of the $600 \mathrm{Mhz}$ oscillation. All of these indicate that the impact ionization can not be the single cause of TAoscillations. Additionally to the IMPATT, the PETT mechanism is also involved in the TA-oscillation because of the remaining plasma behind the SCR. Due to the poor efficiency of the PETT mechanism, without IMPATT it need more time to raise the oscillation. 


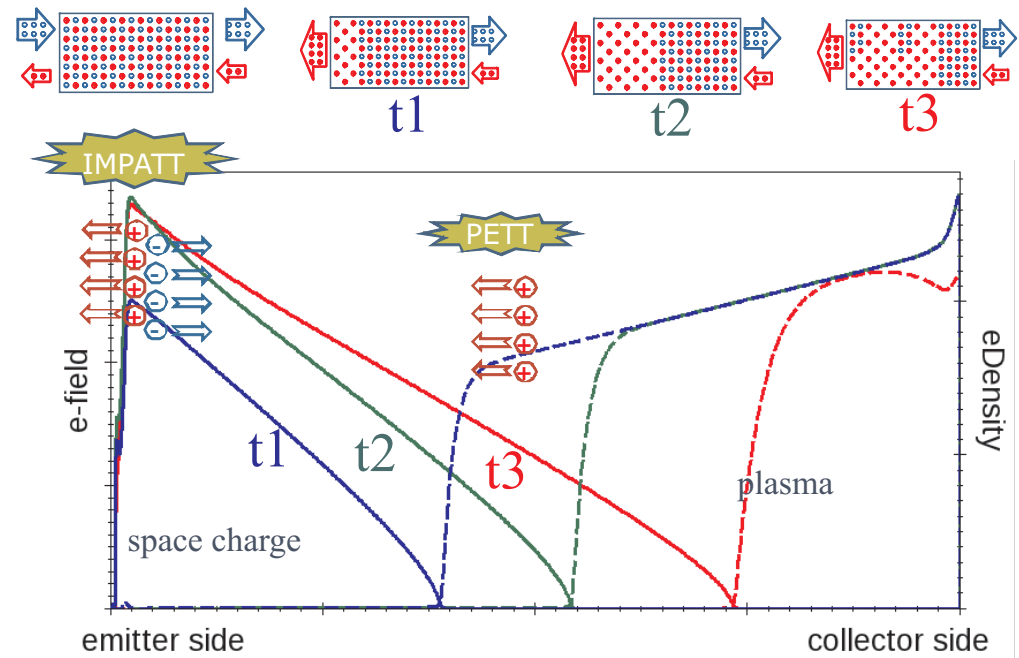

Figure 4.35: Mechanism of TA-oscillation mechanisms

\subsection{Summarized explanation of TA-oscillations}

Fig.4.35 represents the one-dimensional transit time structure during IGBT turn-off. Under forward conduction of the IGBT, the base region is flooded with electrons and holes. After turning-off the MOS-channel, most of the current must be carried by the holes instead of electrons. At that moment, the electron-density decreases and the net hole-density near to the emitter side increases according to the current-share of holes. This results in an net positive charge density (hole-density minus electron-density) near the MOS channel and increases the gradient of electrical field according to the Poisson equation. Due to the high current, the net hole-density is much higher than the doping level. So that, at a $V_{C E}$ far below the block capability, $E_{\max }$ has reached the critical value $E_{C}$ and begins to generate electron-hole pairs at the emitter-side. The so called dynamic avalanche occurs. The electrons generated by avalanche drift through the SCR under the influence of the strong electric field and reach to the anode side. 
Similar to the IMPATT mechanism the transit-time effect hereby gives an oscillation trend to the IGBT chip. The injection-time together with the transit-time of the holes induces a phase shift between the terminal voltage and terminal current in RF-range. At certain frequencies, the IGBT can act as an amplifier. When the addressable resonant circuits are present, oscillations would be raised. Due to the high current, the plasma in the base region is quickly extracted and the $S C R$ is expanding fast to the collector. The expanding of the SCR during the turn-off process disperses the corresponding frequencies of the transit time structure from single frequencies to a continuous frequency band depending on the actual width of the depletion region $\left(W_{S C R}\right)$.

The parasitic components in IGBT modules, for example stray inductance of bond wires and junction capacitance of the free wheel diode can together with the IGBT chip build up resonance circuits. The extension of $S C R$ leads to on one hand the longer transit time of the carriers, on the other hand leads to the reduction of the chip capacitance and therefore the higher eigenfrequencies of the according resonant circuits. If the eigenfrequencies of such resonance circuits match the frequency of the transient-time effect, the Transient Avalanche Oscillation would be raised. Oscillations would occur around the intersections of the eigenfrequencies of the resonant circuit and the efficiency of the amplifier being high.

TA-oscillations are successfully reproduced by device simulations using a two dimension IGBT model which is integrated in a resonant circuit similar to the module setups. Eigenfrequency of the resonant circuit fits the second maximum of IMPATT efficiency resulting in an oscillation around $600 \mathrm{MHz}$. The internal semiconductor states show that the e-field and carriers distributions in the space charge region are overlapped by space waves. The velocities of the waves are appropriate to the corresponding carrier velocities. The IMPATT mechanism is the key player in the TA-oscillation. And additionally, the remaining plasma behind the SCR allows also for 
the plasma extraction transition time (BARITT/PETT) mechanism.

Although TA-oscillations can be raised theoretically at each voltage class of IGBTs. TA-oscillations are until now only observed by IGBTs with over $3.3 \mathrm{kV}$ blocking voltage. The device simulations in this work are based on a 2D half IGBT cell. The filament effects due to the current commutation between the adjacent cell and the paralleled chips can therefore not be investigated. 


\section{Chapter 5}

\section{Countermeasures to \\ Transient Avalanche \\ oscillations}

5.1 Essential measures to suppress TA-oscillations

According to the measurements and analysis in the previous chapters, the dynamic avalanche generation and the transit time effect of the avalanche electrons are identified as the root cause of the TA-oscillation. The high load current density and the switching off of the electron current from the MOS-channel are the key character of the turn off process, in which TAoscillation occurs. The corresponding general possible measures to avoid the TA-oscillations can be summarised as following:

- To apply limitation to the load current and the DC-link voltage so that the dynamic avalanche does not occur. 
- To keep the gate resistor out of the range, in which the TA-oscillation would be raised.

- To avoid the high over voltage by low inductive module design so as to suppress the dynamic avalanche process. This is because that the large stray inductance in the current commutation circuit leads to high over voltage and thus has the similar effect on TA-oscillation as the high DC-link voltage.

- To increase the stray inductance in the resonant circuit for pushing down the resonant frequencies so that they can not be addressed by the transit time effect of the IGBT. Simply, longer emitter wire bonds lead to lower frequency of TA-oscillations. But the high stray inductance would lead to the high over voltage, that the affects the module performance negatively.

- For the investigated $3.3 \mathrm{kV}$ IGBT chip, the TA-oscillations can be avoided by setting up the resonant circuits, so that the resonant frequencies are out of the frequency range from $100 \mathrm{MHz}$ to $800 \mathrm{MHz}$. As the eigenfrequencies and the resonant frequencies change dynamically during the turning off, it is difficult to mismatch the frequencies all the turn-off time. The cross wire bonding between the adjacent IGBTs is effective for the suppression of the low frequency oscillation but not effective for the high frequency TA-oscillation.

- To apply additional electron current from the channel, during the time that TA-oscillation normally happens, is proved to be an effective method against the TA-oscillation. The absence of electrons leads to the high e-field peak near to the emitter side. When the additional electron current is applied from the MOS channel to the SCR, near to the emitter side the derivative $d E / d X$ of the E-field could be reduced and therefore the peak value of the e-field and accordingly the avalanche generation is suppressed. The timing of 
the electron injection should be shortly before the begin of the TAoscillation. The injection should be short enough that the IGBT can be still surely turned off. This method has been realized on a driver concept, which I introduce as next.

\subsection{Driver concept to suppress TA-oscillations - pace maker}

As already mentioned in Chap.4.4, the high total current density and the fast switching off of the channel current lead to the dynamic avalanche during the turning off of the IGBT. On the system level, the load current and the gate resistance can be limited in a certain range, so that the TAoscillation does not happen in the SOA (Safe Operation Area). However, methods to suppress and to avoid TA-oscillation with out restriction of the applications are desired. In this work a driver concept - pace maker [Hon13] is developed to get oscillation free switching performance of IGBT out of the conventional SOA. The basic idea of this driver concept is to apply additional electron current from MOS channel to the SCR during the time that TA-oscillation were found. The additional electrons reduce the net hole density and the gradient of the e-field in the SCR according to Poisson's equation. The maximal e-field and the corresponding avalanche process near to the emitter would be suppressed by the additional channel current. The additional channel current pulse during the tail current has been introduced to suppress the PETT oscillation [Mou01b].

For the conventional IGBT turn off, the driver voltage is pulled down from $+15 \mathrm{~V}$ to $-15 \mathrm{~V}$, and the gate current flows out of the gate to the driver. The gate is therefore discharged. Fig.5.1 shows the pace maker driver concept. After the normal turn off and short time before the dynamic avalanche occurs, an additional positive gate pulse for a few hundreds of $n s$ is applied to the IGBT so that the gate current is forced to change 
its direction. And the gate is charged again for the short time before it ist finally pulled down to $-15 \mathrm{~V}$. A short electron current pulse is injected from the MOS channel to the SCR. As the pulse is short enough, it is not able to turn-on the IGBT completely, but just opening the channel to some extent that dynamic avalanche does not occur. The start condition of the additional pulse can be defined as Eq.5.1. The $V_{C E}$ is compared with the DC-link voltage during the ramp up of $V_{C E}$. The additional gate pulse is triggered as the $V_{C E}$ exceeds the certain ratio of $V_{D C} . \alpha$ and $\beta$ are adjusted by the voltage dividers as shown in Fig.5.2.

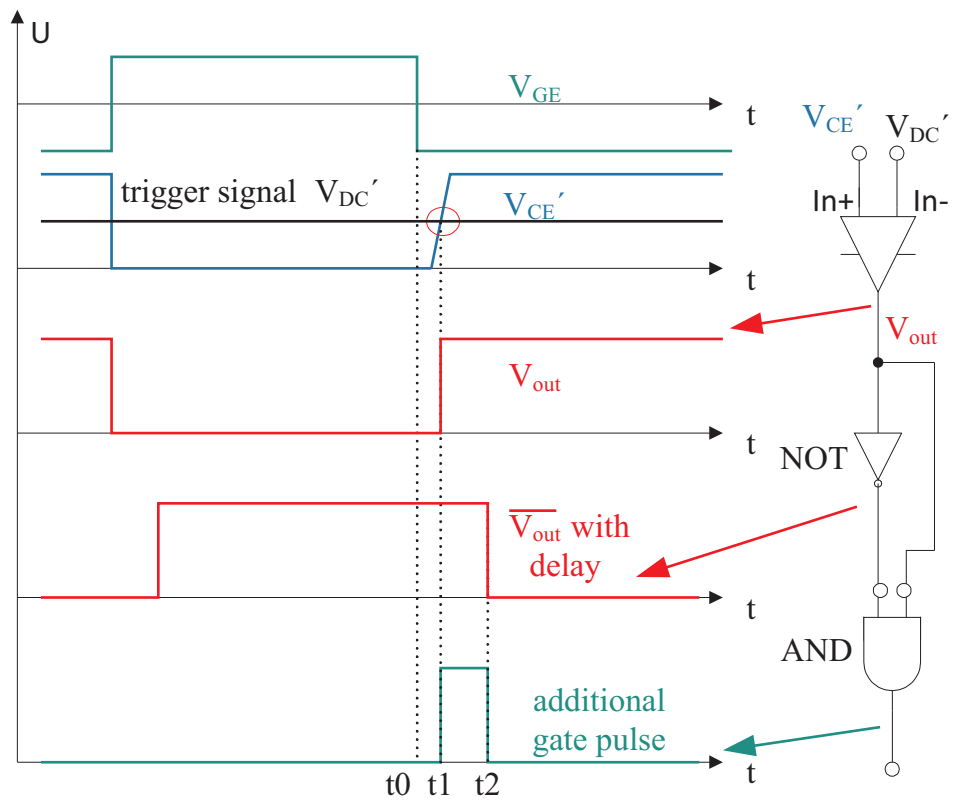

Figure 5.1: Driver concept pace maker 


$$
\begin{aligned}
\alpha V_{C E} & \geq \beta V_{D C} \\
\alpha & =\frac{R 4}{R 3+R 4} \\
\beta & =\frac{R 2}{R 1+R 2}
\end{aligned}
$$

Voltage divider of DC-Link Voltage and

Collector-emitter Voltage
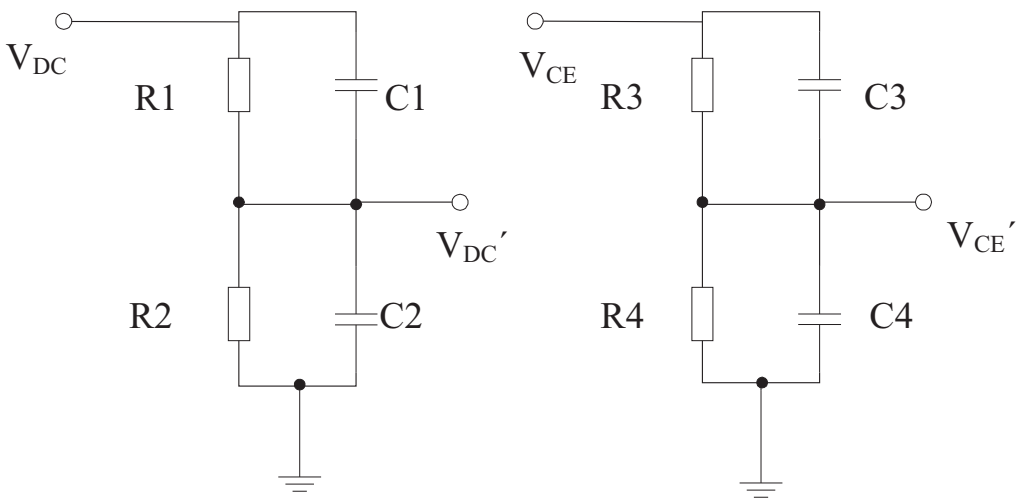

Figure 5.2: Voltage divider on pace maker to define the condition of the additional gate pulse

The additional channel current leads to the reduction of the $E_{\max }$ and causes a reduction of the miller capacitance. The reduction of miller capacitance leads to a negative gate current, which tends to turn off the 


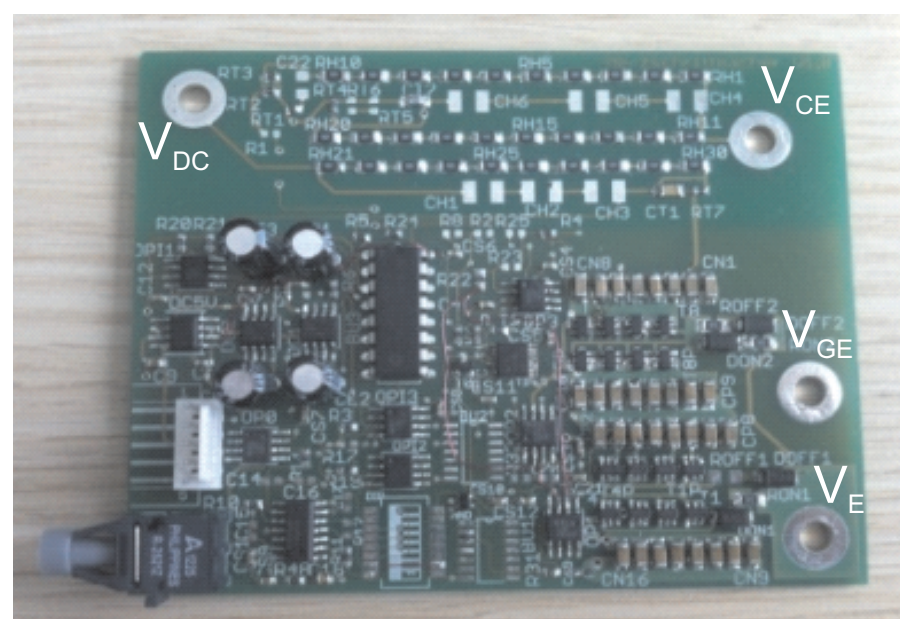

Figure 5.3: Driver concept pace maker

channel again. This so called self turn off effect [Boe14] [Bas14] must be compensated by the pace maker in order to establish the channel current. Measurements to verify the driver concept are processed with the driver board as shown in Fig.5.3. The reference measurement with the additional pulse is shown on the left side of Fig.5.4, on which the TA-oscillation can be observed in the antenna signal. The measurement is repeated with the additional gate pulse and the result can be seen on the right side of Fig.5.4. The TA-oscillation is totally suppressed by the pace maker.

Table 5.1: comparison of the turn off energy $E_{\text {off }}$ of the conventional driver with pace maker at $25^{\circ} \mathrm{C}, I_{L}=1500 \mathrm{~A}$ and $V_{C E}=1800 \mathrm{~V}$ with varying of $R_{G}$

\begin{tabular}{|c|c|c|}
\hline$R_{G}(\Omega)$ & $\begin{array}{c}E_{\text {off }}(\mathrm{mJ}) \\
\text { conventional driver }\end{array}$ & $\begin{array}{c}E_{\text {off }}(\mathrm{mJ}) \\
\text { pace maker }\end{array}$ \\
\hline 2.7 & 2144 & 2240 \\
\hline 1.5 & not allowed & 1907 \\
\hline 1.3 & not allowed & 1848 \\
\hline
\end{tabular}




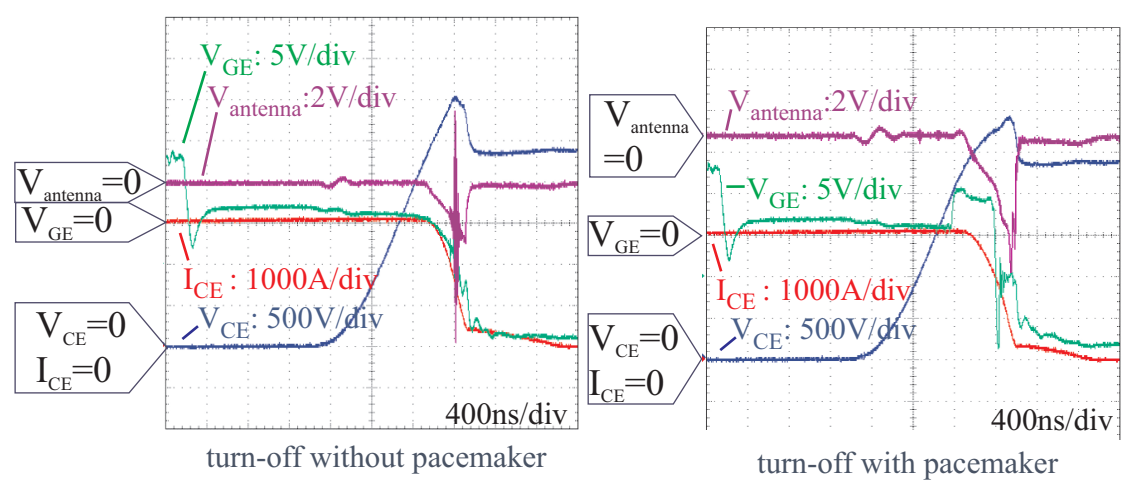

Figure 5.4: Improvement using driver concept pace maker

In the practice, the essential asymmetry and inhomogeneity in the module- and chip-layout is inevitable. Especially during the dynamic process, such as IGBT turn off, such asymmetry and inhomogeneity have generally negative effect on the module performance. Fig.5.5 shows schematically the possible asymmetry within an IGBT module and also within a IGBT chip. During turn off, the gate current flows from gate to the driver. Due to the asymmetry of the parasitic resistance and inductance in the gate circuits, dynamically the gate potential would be different between the chips in a module or between the cells within a chip. This would lead to the asymmetry of the current distribution in the IGBT module and even within a IGBT chip. The pacemaker driver forces a current direction change in the gate circuit. This compensates partially the possible asymmetrical current distribution due to any existing asymmetrical module and chip construction. Besides the suppression of TA-oscillation, the pacemaker concept makes the IGBT even more robust. Generally IGBT is just allowed to be turned off up to two times of the nominal current. If the $I_{C E}$ exceeds this limit, short circuit should be imposed before the IGBT is turned off to avoid the destruction [HB97]. Using the pace maker driver, the $3.3 \mathrm{kV} 1500 \mathrm{~A}$ test module can be successful switched off at 5000A. And 
no limitation of gate resistor is needed any more. Compared to the conventional driver concept the switching energy can also be reduced. For the test module, to avoid the TA-oscillation $2.7 \Omega$ is defined as the lower limit of the SOA. As shown in Tab.5.1, the turn off energy at $2.7 \Omega$ and of the pace maker driver is $2240 \mathrm{~mJ}$, which is $4.5 \%$ slightly higher than the $2144 \mathrm{~mJ}$ of the conventional driver. But with the pace maker oscillation free turn off can be also realized at $R_{G}=1.3 \Omega$. The $E_{\text {off }}$ of $1848 \mathrm{~mJ}$ is then $13.8 \%$ lower than standard.

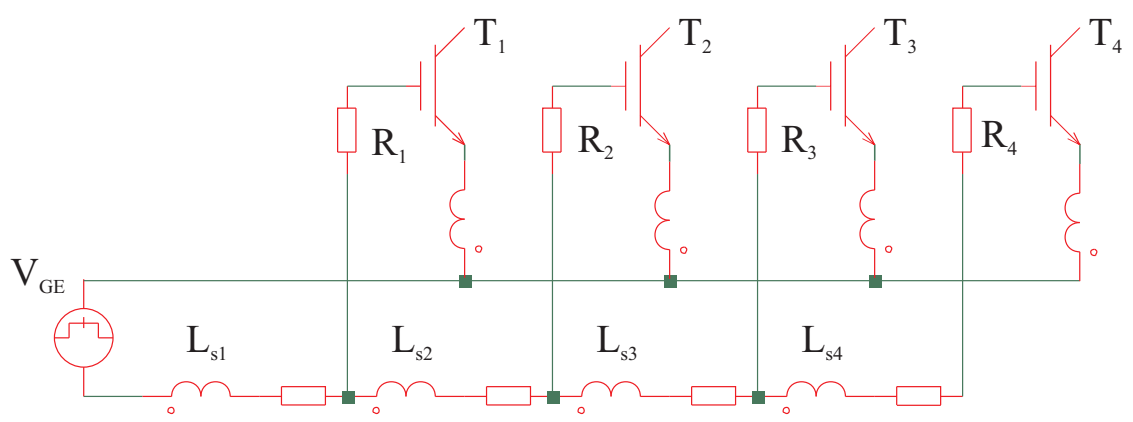

Figure 5.5: Asymmetry of IGBT modules and chips 


\section{Chapter 6}

\section{Summary and outlook}

\subsection{Summary}

Additionally to the PETT oscillation [GMS02] and dynamic IMPATT [LSG98] oscillation, TA-oscillation is found to be a new type HF-oscillation in IGBT modules. TA-oscillations are observed during the raising of the collector voltage and the falling of the collector current, as the dynamic avalanche is ongoing. TA-oscillation is much more dynamic compared to the PETT oscillation and dynamic IMPATT oscillation in diode. The measurements in this thesis show that the gate resistance, the DC-link voltage and the load current are the essential parameter for the TA-oscillation. Although the TA-oscillation is first observed on the gate voltage trace, no active participation of gate can be demonstrated. The HF-oscillation signal on the gate trace is caused by HF-current in the emitter wire bonding through electromagnetic coupling. The TA-oscillation appears just in a certain range of gate resistor while the product of load current and DC-link voltage is significant of set of TA-oscillation, that indicates the correlation of the TA-oscillation to the maximal e-field of the space charge region according to Eq.2.13. And the frequency of the TA-oscillation is found 
between 100 and $800 \mathrm{Mhz}$, that indicates the effect from carrier transit time.

The explanation of the TA-oscillation can be expressed as follows:

Under forward conduction of the IGBT, the base region is flooded with electrons and holes. Due to turn off of the channel, the total load current must be carried by holes. A space charge layer is building up on the emitter side. Due to the high current, the net hole-density is much higher than the doping level. Thus, dynamic avalanche occurs at collector voltage far below the static blocking voltage of the IGBT. Electrons generated by avalanche drift through the space charge region to the collector side similar to the IMPATT diode. The transit time effect causes the chip to act as a high-frequency amplifier. Additionally, the remaining plasma behind the collector side allows also for the plasma extraction transition time (BARITT/PETT) mechanism.

The carrier injection time and the transit time lead to the phase shift between the terminal HF-voltage and the HF-current. According to the conventional consideration, for the IMPATT diode the maximal HF-power generation occurs at the transit angle of $\pi$ for IMPATT diode and $3 / 2 \pi$ for the BARITT diode [Sze81] [EH98]. Till now such consideration is also applied to the frequency estimation of PETT oscillation and dynamic IMPATT oscillations [GMS02] [LSG98] [MD10] [Hee14]. And no consideration are taken for the transit angle above $2 \pi$. With this theory, for a $200 \mu \mathrm{m}$ SCR the transit time frequency can be estimated as around $170 \mathrm{MHz}$ for IMPATT and $240 \mathrm{MHz}$ for PETT/ BARITT mechanisms. This is much lower than the measured oscillation frequencies in this thesis, i.e. $650 \mathrm{MHz}$. As the the power semiconductor chip thickness is not optimized for the RFpower generation, the none ideal situation should also be considered. For the understanding of the amplifier mechanism, the analytical estimation of the HF-energy conversion efficiency of transit time diode of [EH98] is extended in this thesis. The analysis in Chap. 3 shows that additional to 
the optimal transit angle, RF-power generation is also quite possible at the transit angle about $2.9 \pi$ for IMPATT mechanism. Compared with the conventional consideration that corresponds to three times higher IMPATT frequency. The frequency for maximal power generation efficiency is reversely proportional to the width of the space charge region. The expanding of the SCR during turning off of IGBT leads to decreasing of the transit time frequencies. Not only the amplification but also the resonant circuit varies with the expanding of the space charge region. In the IGBT module the resonant circuit is formed out of chip and substrate capacitance as well as the inductance of the wire bonds from IGBT to the diode and diode to the substrate. The diode capacitance is also part of the resonant circuit. The chip capacitance is reversely proportional to the width of the space charge region. During the turning off, the eigenfrequencies of the resonant circuit rise with the expansion of the space charge region. Oscillations would occur around the intersections of the eigenfrequencies of the resonant circuit and the transit time frequencies, that the efficiency of the amplifier being high. Eigenfrequency of the resonant circuit fits with the second maximum of IMPATT efficiency and results in an oscillation around $600 \mathrm{MHz}$.

To reproduce the TA-oscillation, A two dimensional device model of $3.3 \mathrm{kV}$ trench field stop IGBT was setup and integrated in the resonant circuit similar to a IGBT module. The device model and the resonant circuit was again integrated in the test circuit similar to the measurement setup. In contrast to the PETT simulation, TA-oscillations are successfully reproduced without artificial stimulations. The influence of the parameters, such as load current, gate resistance and emitter wire bonds, are verified by the device simulation. The impact ionization shows a periodical process, according to the sine wave of the oscillation. The electrical field has a sinusoidal shape overlapping a triangle, determined by the space charge. Similar wave forms are also found on the electron-distribution and hole- 
distribution in space Charge Region. IMPATT-like mechanism plays the key role in the TA-oscillation. The simulation with deactivated impact ionization shows that in addition to the IMPATT, the PETT mechanism can be also involved in the TA-oscillation.

All the analysis shows that the temporary absence of the electron on the emitter side causes the high E-field peak on the emitter side and leads to TA-oscillation. In this thesis, a driver concept "pace maker"is developed to open the channel again during the time the TA-oscillation normally happens. As the pulse is short enough, it is not able to turn-on the IGBT completely, but just opening the channel to some extent that dynamic avalanche does not occur. Supplying electron current during critical period of time is verified to be a effective method to suppression of dynamic avalanche and the TA-oscillation. The pace maker make the IGBT even more robust because of the compensation of the possible asymmetry in the IGBT module.

\subsection{Outlook}

The one dimension model is used to analyse the RF-power generation efficiency of IMPATT structures analytically. And a narrow avalanche region assumed to inject charge carriers in to the SCR with a delay of $\pi$. During the turn off process of IGBT, the E-field distribution in the SCR changes quickly and this assumption is not always in accordance with the reality and leads to deviation between the frequency estimation and the measurement. Theoretical work can done to develop the more suitable analytical model of the avalanche region during the dynamic changing of E-field distribution. Although the avalanche generation and the electron transit time dominants in TA-oscillation, transit time effect of holes is also found. Further work can be done to investigate the interaction between electrons and holes during TA-oscillations. 
Further research work can be done on the module and chip design of IGBT to improve the dynamic performance. Generally, there are always inevitable asymmetry and inhomogeneities in the IGBT Module, IGBT chip and also within a single cell of the chip. In the conventional design, the IGBT cells are constructed to get a uniform characteristic. Accordingly, the current should be distributed homogeneously. But in reality, a small non-homogeneity and unbalance is unavoidable. And it is generally known that, the slight unavoidable non-homogeneity between and within the IGBT cells may lead to current filaments during the turn off processes of IGBTs [MD10]. This would happen especially under the avalanche condition. The kink on the $V_{C E}$ trace in Fig.4.14 is a sign of current filaments. In the current filaments the local current density can reach up to ten times the average value. Such filaments therefore enhance the transient avalanche dramatically, especially in IGBT for high voltage and high current application, such transient avalanche in IGBTs can become strong enough, so that TA-oscillation takes place and raises EMI problem. Reduction of the density of the current filaments would lead to improved utilization of the IGBT volume. This leads to the idea to reduce the current filament by integration of controlled inhomogeneities into the IGBT chip and into the single cell designs. Further research work can be suggested as follows [Hon14]:

- To investigate the IGBT chip design by arrangement of different (more than two) types of cells within an IGBT chip.

Generally, on the IGBT chip there are large number of IGBT cells with the same cell type implemented. Due to the unavoidable manufacturing tolerances and the asymmetry of the cell arrangement on the chip the switching behaviour of the cells may slightly differ from each other. Since the distribution of such differences cannot be controlled, such minimal difference can lead to strong current filaments. In an IGBT chip, cells can be implemented with differ- 
ent channel widths; different channel contacts, different cell shapes and sizes as well as different doping levels. To generate the nonhomogeneity on IGBT chips by regular or irregular arrangement of various of cell types. Non-homogeneity of the order of regular arrays of various cells of irregular shapes. The target is to suppress the current filament by chip design from the uncontrolled non-homogeneity to a controlled non-homogeneity.

- To investigate the IGBT cell design to integrate inhomogeneity to the gate channel within a IGBT cell.

The lateral non-homogeneity can be realized by deformation of trench gate oxide. Due to the inhomogeneous field distribution in the channel region, during turn-off and before complete channel pinchoff, the channel current can be divided to several sub-streams. The limitation of the current commutation between the adjacent region with in the cell would suppress the stronger filaments and thus the followed avalanche at channel pinch-off.

The trench bottom can also be implemented as wave form or step form, so that the charge commutation from the p-float to the channel region can be homogeneously divided. This would help to suppress TA-oscillations. And there must be other constructions, such as jagged structure, can be introduced to get such effects. 


\section{Appendix A}

\section{Derivation of transit time efficiency}

Here with is the derivation of amplifier efficiency of transit time structures (IMPATT and BARITT). The injection current is consist of three linear functions, which is indeed afflicted with theoretical error. But in case of short injection time this error trend to zero.

$$
\begin{aligned}
P_{D C} & =J_{D C} V_{D C}=\frac{A}{2 \pi} J_{\max } \Theta_{D} V_{D C} \\
P_{R F} & =-\frac{A}{2 \pi} \int_{\Theta_{M}-\Theta_{W} / 2}^{\Theta_{D}+\Theta_{M}+\Theta_{W} / 2} J_{\text {ind }}(\omega t) V_{R F} \sin (\omega t) d(\omega t) \\
& =-\frac{A}{2 \pi}(P 1+P 2+P 3)
\end{aligned}
$$



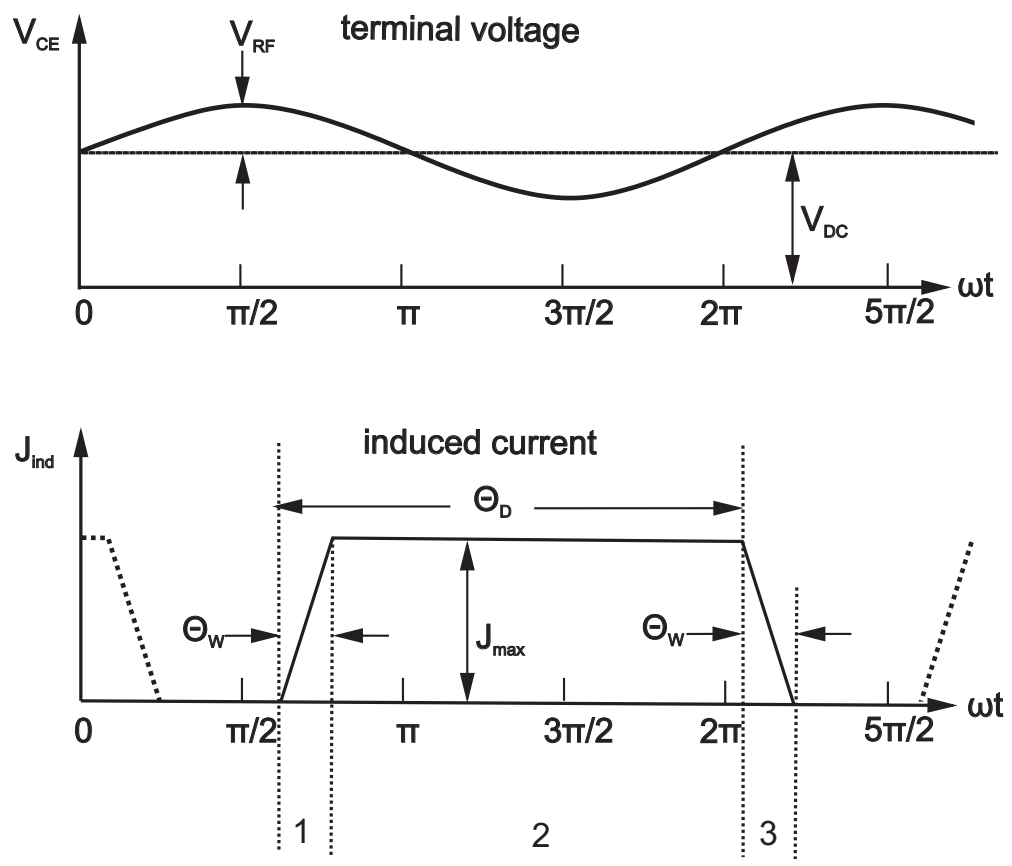

Figure A.1: Diagram of voltage and current in a transit time structure with the simplified charge injection 


$$
\begin{aligned}
P 1 & =\int_{\Theta_{M}-\Theta_{W} / 2}^{\Theta_{M}+\Theta_{W} / 2} J_{i n d}(\omega t) V_{R F} \sin (\omega t) d(\omega t) \\
& =-J_{\text {max }} V_{R F} \cos \left(\Theta_{M}+\Theta_{W} / 2\right)+2 \frac{J_{\max } V_{R F}}{\Theta_{W}} \cos \left(\Theta_{M}\right) \sin \left(\frac{\Theta_{W}}{2}\right) \\
P 2 & =\int_{\Theta_{M}+\Theta_{W} / 2}^{\Theta_{M}+\Theta_{D}-\Theta_{W} / 2} J_{i n d}(\omega t) V_{R F} \sin (\omega t) d(\omega t) \\
& =J_{\text {max }} V_{R F}\left(\cos \left(\Theta_{M}+\Theta_{W} / 2\right)-\cos \left(\Theta_{M}+\Theta_{D}-\Theta_{W} / 2\right)\right) \\
P 3 & =\int_{\Theta_{M}+\Theta_{D}-\Theta_{W} / 2}^{\Theta_{M}+\Theta_{D}+\Theta_{W} / 2} J_{i n d}(\omega t) V_{R F} \sin (\omega t) d(\omega t) \\
& =J_{\text {max }} V_{R F} \cos \left(\Theta_{M}+\Theta_{D}-\Theta_{W} / 2\right) \\
& +\frac{J_{\max } V_{R F}}{\Theta_{W}}\left(\sin \left(\Theta_{M}+\Theta_{D}+\Theta_{W} / 2\right)-\sin \left(\Theta_{M}+\Theta_{D}-\Theta_{W} / 2\right)\right) \\
P_{R F} & =-\frac{A}{2 \pi} J_{m a x} V_{R F} \frac{\sin \left(\Theta_{W} / 2\right)}{\Theta_{W} / 2}\left(\cos \left(\Theta_{M}+\Theta_{D}\right)-\cos \left(\Theta_{M}\right)\right) \\
P_{D C} & P_{R F}-\frac{V_{R F}}{V_{D C}} \frac{\sin \left(\frac{\Theta_{W}}{2}\right)}{\frac{\Theta_{W}}{2}} \frac{\cos \left(\Theta_{M}+\Theta_{D}\right)-\cos \Theta_{M}}{\Theta_{D}} \\
& \\
&
\end{aligned}
$$




$$
\begin{aligned}
& \eta_{1}\left(\Theta_{W}\right)=\frac{\sin \left(\frac{\Theta_{W}}{2}\right)}{\frac{\Theta_{W}}{2}} \\
& \eta_{2}\left(\Theta_{D}\right)=\frac{\cos \left(\Theta_{M}+\Theta_{D}\right)-\cos \Theta_{M}}{\Theta_{D}}
\end{aligned}
$$




\section{Appendix B}

\section{Test box and the RF-noise problems}

At begin of the investigation on TA-oscillation, HF-disturbance at about $600 \mathrm{MHz}$ is some time observed on the trace of load current, as shown in Fig.B.1. But the load current is measured by a Person coil, whose response frequency is under $30 \mathrm{MHz}$. The capacitive coupling in this case is responsible for disturbance. Fig.B.2 shows the schematic of the test setup. The impedance of the GND conductor between the test box and the oscilloscope, the impedance of the GND conductor of the BNC cabel between Person current sense and the coupling capacitance of Persen current sense to the test box built a closed loop, that capture the HF-oscillation signal similarly to an antenna. The HF-signal measured at the oscilloscope is the voltage drop on the BNC cable of the Person current sensor according to the voltage divider as shown in Fig.B.2, but not the current signal. This effect can be eliminated by enlarging the impedance of the GND conductor between the test box and the oscilloscope. 


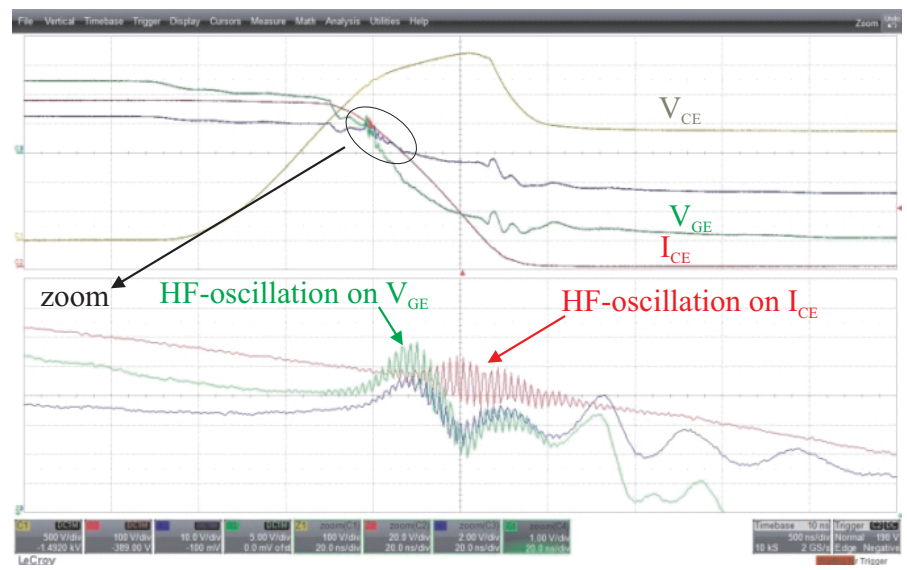

Figure B.1: oscillation signal observed on the load current

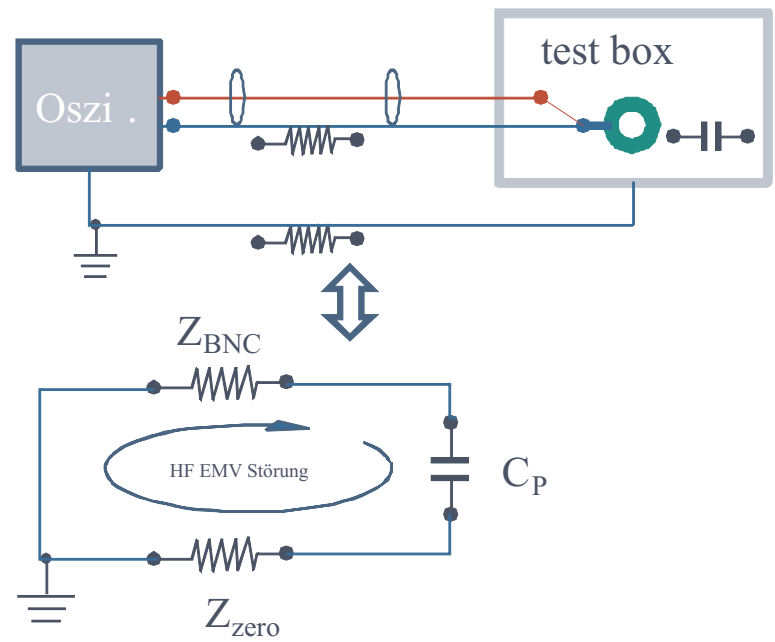

$\mathrm{C}_{\mathrm{P}}$ : capacitivecupling between Person current sensor and test box

$Z_{\mathrm{GND}}$ : impedance of the GND conductor between test box and oscilloscope

$Z_{\mathrm{BNC}}$ : impedance of the BNC cable from Person current sensor to oscilloscope

Figure B.2: Schematic of capacitive cupling and the voltage divider in the test setup 


\section{Bibliography}

[ABG ${ }^{+}$99] Auerbach, F. ; Bauer, J.G ; Glantschnig, M.; Gottert, J. ; Hierholzer, M. ; Porst, A.; Reznik, D. ; Schulze, H. ; Schulze, T. ; Spanke, R.: 6.5 kV IGBT-modules. In: Industry Applications Conference 3 (1999), Nr. 34, S. 17701774

[Bal79] Baliga, B. J.: Enhancement and depletion mode veritcal channel MOS gate thyristor. In: Electronic Letters 15 (1979), September, Nr. 20, S. 645-647

[Bas14] Basler, Thomas: Ruggedness of High-Voltage IGBTs and Protection Solutions, Technische Universität ChemnitzUniversity Bremen, Dissertation, 2014

[BD10] Bayerer, R. ; Domes, D.: Power circuit design for clean switching. In: 6th IEEE International Conference on Integrated Power Electronics Systems (CIPS) (2010), S. 1-6

[Boe14] Boehmer, Juergen W.: Wirkung des Ladungsträgerplasmas auf das Abschaltenverhalten von Insulated Gate Bipolar Transistoren, University Rostock, Dissertation, Februar 2014 
[BS67] BENDA, HANSJOCHEN ; SPENKE, EBERHARD: Reverse recovery processes in silicon power rectifiers. In: Proceedings of the IEEE 55 (1967), August, Nr. 8, S. 1331-1354

[BW80] Becke, Hans W. ; Wheathley, C. Frank J.: Power MOSFET with an anode region. In: U.S. patent No. 4,364,073 (1980), Nov.

[CMMO75] Canali, C. ; Majni, G.; Minder, R.; Ottaviani, G.: Electron and hole drift velocity measurements in silicon and their empirical relation to electric field and temperature. In: Electron Devices, IEEE Transactions 22 (1975), November, S. $1045-1047$

[DLS03] DomeiJ, M. ; Lutz, J. ; Silber, D.: On the destruction limit of Si power diodes during reverse recovery with dynamic avalanche. In: IEEE TRANSACTIONS ON ELECTRON DEVICES 50 (2003), Nr. 2, S. 486-493

[EH98] Eisele, Heribert ; Harddad, George I.: Active microwave diodes. In: Sze, SM (Hrsg.): Modern Semiconductor Device Physics. New York : John Wiley \& Sons, 1998, Kapitel 6, S. 343-407

[FHK09] Fujita, Shigeto ; Hussein, Khalid H. ; Kitamura, Shuichi: Investigation on IGBT High-Frequency Plasma. In: IEEE TRANSACTIONS ON ELECTRON DEVICES 24 (2009), Juni, Nr. 6, S. 1570-1576

[GH66] Gelden, M. ; Hines, E.: Electronic Tuning Effects in the Read Microwave Avalanche Diode. In: IEEE TRANSACTIONS ON ELECTRON DEVICES 13 (1966), Januar, Nr. 1, S. $169-175$ 
[GMS01] Gutsmann, B. ; Mourick, P. ; Silber, D.: Hochfrequenzoszillationen im IGBT-Schweifstrom. In: HalbleiterLeistungsbauelemente und ihre systemtechnische Anwendung (2001), Nr. 30

[GMS02] Gutsmann, B. ; Mourick, P. ; Silber, D.: Plasma extraction transit time oscillations in bipolar power devices. In: Solid State Electron. 46 (2002), April, Nr. 5, S. 133-138

[Gut06] Gutsmann, Berndt: Charakterisierung und Einfluss parasitrer elektromagnetischer Effekte in Leistungselektronischen Systemen, University Bremen, Dissertation, August 2006

[HB97] Hierholze, M. ; BAyerer, R.: Improved Characteristics of $3.3 \mathrm{kV}$ IGBT Modules. In: PCIM (1997), Nr. 31. - Nürnber Germany

[Hee14] HEEB, Michael: Modellierung der parasitären passiven Elemente in IGBT-Hochleistungsmodulen, University Kassel, Dissertation, April 2014

[Hon13] Hong, T.: Ansteuerschaltung für Leistungshalbleiter. In: Deutsche Patentanmeldung DE 102013233135 (2013), Nov.

[Hon14] Hong, T.: Inhomogenous Power Semiconductor Devices. In: United States Patent Application Publication US20140131766A1 (2014), May

[HPB $\left.{ }^{+} 14\right]$ Hong, T. ; Pfirsch, F. ; Bayerer, R. ; Lutz, J. ; Silber, D.: Tansient Avalanche Ocillation of IGBTs Under high Current ISPSD, 2014 (Proceedings of International Symposium on Power Semiconductor Devices \& IC's, Waikoloa, Hi 26), S. $355-358$ 
[KOH ${ }^{+}$93] Kitagawa, M. ; Omura, I. ; Hasegawa, S. ; Inoue, T. ; NAKAGAWA, A.: A 4500V Injektion enhanced Insulated gate bipolar Transistor (IEGT) Operating in a Mode Similar to a Thyristor. In: Electron Devices Meeting, 1993. IEDM '93. Technical Digest., International (1993), S. 679-682

[LMP $\left.{ }^{+} 00\right]$ Laska, T. ; MÜnzer, M. ; Pfirsch, F. ; Schäffer, C. ; SchmidT, T.: The Field Stop IGBT (FS IGBT). A new power device concept with a great improvement potential. Toulouse, Mai 2000 (Proceedings of International Symposium on Power Semiconductor Devices \& IC's 12), S. 355-358

[LS94] Lutz, J. ; Scheuermann, U.: Advantages of the new controlled axial lifetime diode. In: PCIM (1994), Nr. 28. Nürnberg Germany

[LSG98] Lutz, J. ; SÜDKAmp, W. ; Gerlach, W.: IMPATT oscillations in fast recovery diodes due to temporarily charged radiation-induced deep levels. In: Solid-State Electronics 42 (1998), Juni, Nr. 6, S. 931-938

[LSSD11] Lutz, Josef ; Schlangenotto, Heinrich ; Scheuermann, Uwe ; Doncker, Rik D.: Semiconductor Power Devices. 1. Springer Heiderberg Dordrecht London New York, 2011

[Lut00] LuTz, J.: Freilaufdiode für schnell schaltende Leistungsbauelemente, TU Ilmenau, Dissertation, 2000

[Lut06] Lutz, Josef: Halbleiter-Leistungsbauelemente. Berlin Heidelberg : Springer-Verlag, 2006

[MD10] MÜller-Dauch, Andreas: Simulationsuntersuchungen zur Grenzbelastbarkeit von IGBTs im Abschaltvorgang, University Bremen, Dissertation, Dezember 2010 
[MGS02] Mourick, P. ; Gutsmann, B. ; Silber, D.: Ultra high frequency oscillations in the reverse recovery current of fast diodes. Santa FA NM, Juni 2002 (Proceedings of International Symposium on Power Semiconductor Devices \& IC's 14), S. $205-208$

[Mou01a] Mouick, P.: Method of driving power semiconductor modules. In: Deutsche Patentschrift DE10158374C1 (2001), Nov.

[Mou01b] Mouick, P.: Power semiconductor module control method provides suppression of plasma extraction transit time oscillations using additional control pulse. In: Deutsche PatentschriftDE10156618 C1 (2001), Nov.

[MRP05] Kap. 2.5 In: Martin R. Parker, William E. W.: The Electronics Handbool. CRC Pressp, 2005

[OFO99] Omura, I. ; Fichtner, W. ; Ohashi, H.: Oscillation Effects in IGBT's Related to Negative Capacitance Phenomena. In: IEEE TRANSACTIONS ON ELECTRON DEVICES 46 (1999), Januar, Nr. 1, S. 237-243

[OOSO97] Omura, I. ; Ogura, T. ; Sugiyama, K. ; Ohashi, H.: Carrier injection enhancement effect of high voltage MOS devices- Device physics and design concept. In: IEEE TRANSACTIONS ON ELECTRON DEVICES (1997), Mai, S. 217-220

[Pau81] PAUL, Reinhold: Halbleitersonderbauelement Höchstfrquenzund Leistungsbauelemente. Berlin : VEB VERLAG TECHNIK, 1981 (Elektronische Festkörperbauelemente)

[Pfi12] Pfirsch, F.: private communication 13.03.2012, Diss., 2012 
[R.B10] R.BAYERER: IGBT-driver circuit for desaturated turn-off with high desaturation level. In: Patentschrift US 7768337 B2 (2010)

[RKK $\left.{ }^{+} 02\right]$ Rahimo, M. ; Kopta, A. ; Kaminski, S. Eicherand N. ; Bauer, F. ; Schlapbach, U. ; Linder, S.: Extending the Boundary Limits of High Voltage IGBTs and diodes to above $8 \mathrm{kV}$. In: International Symposium on Power Semiconductor Devices ES IC's (2002), Nr. 14, S. 41-44

[Roe77] VAn DE Roer, T.G.: D.C and small-signal A.C. propertiew of Silicon BARITT diodes, University of Eindhoven, Dissertation, Januar 1977

[SGMM02] Silber, D. ; Gutsmann, B. ; Mourick, P. ; Miller, G.: Semiconductor Component circuit with a reduced oscillation tendency. In: Internationale Anmeldung WO 03/049185 A2 (2002), Nov.

[SGMm03] Silber, D. ; Gutsmann, B. ; Mourick, P. ; Miller, G.: Halbleiterbauelementanordnung mit verminderter Oszillationsneigung. In: Deutsche Patentanmeldung DE 10159851 A1 (2003), Jun.

[Sho38] Shockley, William: Currents to Conductors Induced by a Moving Point Charge. In: Journal of Applied Physics 9 (1938), Oktober, S. 635

[Sil05] SilBE, D.: Halbleiterbauelement, University Bremen, Vorlesungsskript, 2005

[Sil14] Silbe, D.: private communication 18.09.2014, Diss., 2014

[SL04] Siemieniec, R. ; Lutz, J.: Possibilities and limits of axial lifetime control by radiation induced centres in fast recovery 
diodes. In: Microelectronics Journal 35 (2004), Nr. 3, S. 259-267

[SLH04] Siemieniec, R. ; Lutz, J. ; Herzer, R.: Analysis of dynamic impatt oscillations caused by radiation induced deep centers with local and homogenous vertical distribution. In: IEE Proc.-Circuits Devices Syst. 151 (2004), Nr. 3, S. 219-224

[SLNM03] Siemieniec, R. ; Lutz, J. ; Netzel, M. ; Maurick, P.: Transit time oscillations as a source of EMC problems in bipolar power devices. In: EPE TRANSACTIONS ON ELECTRON DEVICES (2003)

[SMnl06] Siemieniec, Ralf ; Mourick, Paul ; Netzel, Mario ; Lutz, Josef: The Plasma Extraction Transit-Time Oscillation in Bipolar Power Devices - Mechanism, EMC Effects, and Prevention. In: IEEE TRANSACTIONS ON ELECTRON DEVICES 53 (2006), Februar, Nr. 2, S. 369-379

[SN89] Schlangenotto, H. ; Neubrand, H.: Dynamischer Avalanche beim Abschalten von GTO-Thyristoren und IGBTs. In: Arch. Elektrotechnik 72 (1989), S. 113

[SSL02a] Siemieniec, R. ; SÜDKAmp, W. ; Lutz, J.: APPLYING DEVICE SIMULATION FOR LIFETIME-CONTROLLED DEVICES. In: Fourth IEEE International Caracas Conference on Devices, Circuits and Systems, Aruba (2002)

[SSL02b] Siemieniec, R. ; Südkamp, W. ; Lutz, J.: Determination of parameters of radiation induced traps in silicon. In: SolidState Electronicsl 46 (2002), Nr. 2, S. 891-901

[syn11] SYNOPSYS: TCAD Sentaurus Version F-2011.09. 2011 
[Sze81] SzE, SM: Physics of Semiconductor Devices. 2. Edition. New York : John Wiley \& Sons, 1981

[TKY ${ }^{+}$98] Takahashi, Y. ; Koga, T. ; Yamasaki, K. ; Kirihata, H. ; Seki, Y. ; Eschrich, F.: 2.5kV/1800A Power Pack IGBT. Bad Nauheim, Mai 1998 (ETG - Fachbericht 72), S. 43-54

[TYK ${ }^{+}$97] Takahashi, Y. ; Yoshikawa, K. ; Koga, T. ; Soutome, M. ; Takano, T. ; Kirihata, H. ; Seki, Y.: Ultra high-power 2.5kV-1800A Power Pack IGBT. Weimar, Mai 1997 (Power Semiconductor Devices and IC's, 1997. ISPSD '97., 1997 IEEE International Symposium on 26), S. 233-236

[ZS95] Zimmermann, W. ; Sommer, K.: LeistungshalbleiterModul mit parallelgeschalteten IGBT-Chips and zusätzlicher Verbindung der Emitterkontakte. In: Patentschrift DE 19549011C2 (1995) 
